Supporting Information to Accompany:

\title{
Enantioselective Hydrogenation of N-H Imines
}

Guohua Hou, ${ }^{\dagger}$ Francis Gosselin, ${ }^{*}{ }^{\star}$, Wei Li,${ }^{\dagger}$ J. Christopher McWilliams, ${ }^{\dagger}$ Yongkui Sun,${ }^{\ddagger}$ Mark Weisel, ${ }^{\ddagger}$ Paul D. O'Shea, ${ }^{\S}$ Cheng-yi Chen,${ }^{\ddagger}$ Ian W. Davies, ${ }^{\ddagger}$ and Xumu Zhang ${ }^{* \dagger}$

Department of Process Research, Merck Research Laboratories, P.O. Box 2000, Rahway, NJ 07065, Department of Process Research, Merck Frosst Centre for Therapeutic Research, 16711 route trans-canadienne, Kirkland, Québec, Canada H9H 3L1, and Department of Chemistry, Rutgers, The State University of New Jersey, 610 Taylor Road, Piscataway, NJ 08854-8066

\section{(A) Preparation and Physical Data for $\mathbf{N}-\mathrm{H}$ imines}

(B) General Procedure for Asymmetric Hydrogenation of $\mathrm{N}-\mathrm{H}$ imines

Hydrochloride .56

(C) Analytical Data for the Hydrogenation Products ..............................S6

(D) Imine Deuteration experiment..............................................S11

(E) NMR Spectra of new products ..............................................S12

(F) GC Charts for Hydrogenation Products .....................................S25

General: Unless otherwise noted all reactions were run under a nitrogen atmosphere, solvents and reagents were transferred by syringe. All manipulations involving $[\operatorname{Ir}(\mathrm{COD}) \mathrm{Cl}]_{2}$ and $(S, S)$-f-binaphane were performed in a $\mathrm{N}_{2}$-filled glove-box. NMR spectra were recorded with a Varian spectrometer at $400 \mathrm{MHz}\left({ }^{1} \mathrm{H} \mathrm{NMR}\right)$ and $100 \mathrm{MHz}\left({ }^{13} \mathrm{C} \mathrm{NMR}\right)$ or $500 \mathrm{MHz}\left({ }^{1} \mathrm{H} \mathrm{NMR}\right)$ and $125 \mathrm{MHz}\left({ }^{13} \mathrm{C} \mathrm{NMR}\right)$ in $\mathrm{CD}_{3} \mathrm{OD}$, DMSO- $d_{6}$ or $\mathrm{CDCl}_{3}$. Chemical shifts were reported in ppm downfield from internal $\mathrm{Me}_{4} \mathrm{Si}$. Optical rotations were determined using a Perkin Elmer 341 MC polarimeter. HRMS were recorded on a Thermo LTQ Orbitrap hybrid mass spectrometer. GC analyses were performed using Hewlett Packard Model HP 7890 Series. The N-H imines salts were prepared by organometallic addition to nitriles.

\section{(A) Preparation and Physical Data for $\mathrm{N}-\mathrm{H}$ Imine Hydrochloride Salts}

Representative procedure: A round-bottom flask was charged with nitrile $(50.0 \mathrm{mmol})$ and THF $(50 \mathrm{~mL})$. The mixture was cooled to $-78{ }^{\circ} \mathrm{C}$ and $\mathrm{MeLi}(50.0 \mathrm{~mL}, 1.6 \mathrm{M}$ in diethyl ether) was added dropwise over $1 \mathrm{~h}$. After addition, the resulting mixture was stirred for $2 \mathrm{~h}$ and quenched with anhydrous $\mathrm{MeOH}(12 \mathrm{~mL})$. The mixture was then stirred at $\mathrm{rt}$ for $2 \mathrm{~h}$. The suspension was filtered on Solka-Floc or Celite and the filtrate was concentrated under vacuum. The residue was dissolved in MTBE $(50 \mathrm{~mL})$ and treated with $\mathrm{HCl} / \mathrm{Et}_{2} \mathrm{O}(50.0 \mathrm{~mL}, 1 \mathrm{M})$. The slurry was stirred for $30 \mathrm{~min}$ and filtered to obtain the product as free-flowing off-white to yellow solids. 
<smiles>CC(=[NH2+])c1ccc(C)cc1</smiles>

1-p-Tolylethaniminium chloride (3a): Yield: 98\%. ${ }^{1} \mathrm{H}$ NMR $\left(\mathrm{CD}_{3} \mathrm{OD}, 500 \mathrm{MHz}\right) \delta 8.00(\mathrm{~d}$, $2 \mathrm{H}, J=8.0 \mathrm{~Hz}, \mathrm{Ar}-\mathrm{H}), 7.51$ (d, $2 \mathrm{H}, J=8.0 \mathrm{~Hz}, \mathrm{Ar}-\mathrm{H}), 2.90$ (s, 3H, $\left.\mathrm{CH}_{3}\right), 2.49$ (s, 3H, $\left.\mathrm{CH}_{3}\right)$;

${ }^{13} \mathrm{C} \mathrm{NMR}\left(\mathrm{CDCl}_{3}, 125 \mathrm{MHz}\right): \delta 186.7,148.7,130.3,129.5,127.5,20.9,20.7$; HRMS (EI) Calcd for $\mathrm{C}_{9} \mathrm{H}_{12} \mathrm{~N}$ : 134.0970. Found: 134.0964 .<smiles>CC(=[NH2+])c1ccccc1</smiles>

1-Phenylethaniminium chloride (3b) ${ }^{1}$ : Yield: $96 \% .{ }^{1} \mathrm{H}$ NMR $\left(\mathrm{CD}_{3} \mathrm{OD}, 400 \mathrm{MHz}\right) \delta 8.07-8.04$ (m, 2H, Ar-H), 7.86-7.82 (m, 2H, Ar-H), 7.70-7.65 (m, 2H, Ar-H), 2.91 (s, 3H, $\mathrm{CH}_{3}$ ).<smiles>CCC(=[NH2+])c1ccccc1</smiles>

1-Phenylpropan-1-iminium chloride (3c) ${ }^{2}$ : Yield: 94\%. ${ }^{1} \mathrm{H}$ NMR (DMSO- $\left.d_{6}, 400 \mathrm{MHz}\right) \delta$ 12.44 (bs, 2H, NH 2$), 8.04$ (d, 2H, J=8.0 Hz, Ar-H), 7.79 (t, 1H, J=7.2 Hz, Ar-H), 7.64 (t, 2H, $J$ $=7.2 \mathrm{~Hz}, \mathrm{Ar}-\mathrm{H}), 3.20\left(\mathrm{dd}, 2 \mathrm{H}, J=7.2\right.$ and $\left.14.8 \mathrm{~Hz}, \mathrm{CH}_{2}\right), 1.19\left(\mathrm{t}, 3 \mathrm{H}, J=7.2 \mathrm{~Hz}, \mathrm{CH}_{3}\right)$.<smiles>CCCCC(=[NH2+])c1ccccc1</smiles>

1-Phenylpentan-1-iminium chloride (3d): Yield: 95\%. ${ }^{1} \mathrm{H}$ NMR (DMSO- $\left.d_{6}, 400 \mathrm{MHz}\right) \delta 12.77$ (bs, 2H, $\mathrm{NH}_{2}$ ), 8.12-8.10 (m, 2H, Ar-H), 7.85-7.81 (m, 1H, Ar-H), 7.70-7.66 (m, 2H, Ar-H), $3.24\left(\mathrm{t}, 2 \mathrm{H}, J=7.6 \mathrm{~Hz}, \mathrm{CH}_{2}\right), 1.61-1.53\left(\mathrm{~m}, 2 \mathrm{H}, \mathrm{CH}_{2}\right), 1.40-1.31\left(\mathrm{~m}, 2 \mathrm{H}, \mathrm{CH}_{2}\right), 0.89$ (t, $3 \mathrm{H}, J=$ $\left.7.6 \mathrm{~Hz}, \mathrm{CH}_{3}\right)$.<smiles>CC(C)(C)C(=[NH2+])c1ccccc1</smiles>

2,2-Dimethyl-1-phenylpropan-1-iminium chloride (3e): Yield: 90\%. ${ }^{1} \mathrm{H}$ NMR (DMSO- $d_{6}, 400$ $\mathrm{MHz}) \delta 12.74$ (bs, 2H, NH ), 7.66-7.61 (m, 1H, Ar-H), 7.56-7.55 (m, 4H, Ar-H), 1.33 (s, 9H, $\left.\mathrm{CH}_{3}\right)$.<smiles></smiles>

1-(4-Methoxyphenyl)ethaniminium chloride (3f): Yield: 98\%. ${ }^{1} \mathrm{H}$ NMR (DMSO- $\left.d_{6}, 400 \mathrm{MHz}\right)$ $\delta 12.23$ (bs, 2H, NH${ }_{2}$ ), 8.17 (d, 2H, $J=9.2 \mathrm{~Hz}, \mathrm{Ar}-\mathrm{H}$ ), 7.18 (d, 2H, J=8.8 Hz, Ar-H), 3.89 (s, 3H, $\left.\mathrm{OCH}_{3}\right), 2.79\left(\mathrm{~s}, 3 \mathrm{H}, \mathrm{CH}_{3}\right) ;{ }^{13} \mathrm{C} \mathrm{NMR}\left(\mathrm{CD}_{3} \mathrm{OD}, 125 \mathrm{MHz}\right): \delta 184.3,166.9,132.5,121.9,115.2$, 
55.7, 20.7; HRMS (EI) Calcd for $\mathrm{C}_{9} \mathrm{H}_{12} \mathrm{NO}$ : 150.0919. Found: 150.0913.<smiles>CC(=[NH2+])c1ccc(F)cc1</smiles>

1-(4-Fluorophenyl)ethaniminium chloride (3g): Yield: 99\%. ${ }^{1} \mathrm{H}$ NMR (DMSO- $\left.d_{6}, 400 \mathrm{MHz}\right) \delta$ 12.63 (bs, 2H, $\left.\mathrm{NH}_{2}\right), 8.25-8.21$ (m, 2H, Ar-H), 7.55-7.50 (m, 2H, Ar-H), $2.83\left(\mathrm{~s}, 3 \mathrm{H}, \mathrm{CH}_{3}\right) ;{ }^{13} \mathrm{C}$ NMR $\left(\mathrm{CD}_{3} \mathrm{OD}, 125 \mathrm{MHz}\right): \delta 186.1,168.7,166.7,133.0,132.9,126.9,126.8,117.0,116.8,21.4$; HRMS (EI) Calcd for $\mathrm{C}_{8} \mathrm{H}_{9} \mathrm{NF}: 138.0719$. Found: 138.0714 .<smiles>CC(=[NH2+])c1ccc(Cl)cc1</smiles>

1-(4-Chlorophenyl)ethaniminium chloride (3h): Yield: 98\%. ${ }^{1} \mathrm{H}$ NMR (DMSO- $\left.d_{6}, 400 \mathrm{MHz}\right)$ $\delta 12.66$ (bs, 2H, NH ), 8.11 (d, 2H, J=8.8 Hz, Ar-H), 7.75 (d, 2H, J=8.8 Hz, Ar-H), 2.83 (s, 3H, $\left.\mathrm{CH}_{3}\right) ;{ }^{13} \mathrm{C}$ NMR $\left(\mathrm{CD}_{3} \mathrm{OD}, 125 \mathrm{MHz}\right): \delta 186.7,142.7,131.1,129.9,129.1,21.4$; HRMS (EI) Calcd for $\mathrm{C}_{8} \mathrm{H}_{9} \mathrm{NCl}: 154.0424$. Found: 154.0418 .<smiles>CC(=[NH2+])c1ccc(Br)cc1</smiles>

1-(4-Bromophenyl)ethaniminium chloride (3i): Yield: 80\%. ${ }^{1} \mathrm{H}$ NMR $\left(\mathrm{CD}_{3} \mathrm{OD}, 400 \mathrm{MHz}\right) \delta$ $7.98(\mathrm{~d}, 2 \mathrm{H}, J=9.2 \mathrm{~Hz}, \mathrm{Ar}-\mathrm{H}), 7.88-7.84(\mathrm{~m}, 2 \mathrm{H}, \mathrm{Ar}-\mathrm{H}), 2.90\left(\mathrm{~s}, 3 \mathrm{H}, \mathrm{CH}_{3}\right) ;{ }^{13} \mathrm{C} \mathrm{NMR}\left(\mathrm{CD}_{3} \mathrm{OD}\right.$, $100 \mathrm{MHz}$ ): $\delta$ 187.0, 148.7, 132.9, 132.0, 130.9, 129.9, 21.4; HRMS (EI) Calcd for $\mathrm{C}_{8} \mathrm{H}_{9} \mathrm{NBr}$ : 197.9918. Found: 197.9913.<smiles>CC(=[NH2+])c1ccc(C(F)(F)F)cc1</smiles>

1-(4-(Trifluoromethyl)phenyl)ethaniminium chloride (3j): Yield: 81\%. ${ }^{1} \mathrm{H}$ NMR (DMSO- $d_{6}$, $400 \mathrm{MHz}) \delta 8.11(\mathrm{~d}, 2 \mathrm{H}, J=8.0 \mathrm{~Hz}, \mathrm{Ar}-\mathrm{H}), 7.86(\mathrm{~d}, 2 \mathrm{H}, J=8.0 \mathrm{~Hz}, \mathrm{Ar}-\mathrm{H}), 2.83\left(\mathrm{~s}, 3 \mathrm{H}, \mathrm{CH}_{3}\right)$;<smiles></smiles>

1-m-Tolylethaniminium chloride (3k): Yield: 95\%. ${ }^{1} \mathrm{H}$ NMR (DMSO-d $\left.d_{6}, 400 \mathrm{MHz}\right) \delta 12.60$ (bs, 2H, NH $\mathrm{NH}_{2}, 7.95$ (s, 1H, Ar-H), 7.91 (d, 1H, J=8.0 Hz, Ar-H), 7.62 (d, 1H, J=8.0 Hz, Ar-H), $7.53(\mathrm{t}, 1 \mathrm{H}, J=8.0 \mathrm{~Hz}, \mathrm{Ar}-\mathrm{H}), 2.83\left(\mathrm{~s}, 3 \mathrm{H}, \mathrm{CH}_{3}\right), 2.39\left(\mathrm{~s}, 3 \mathrm{H}, \mathrm{CH}_{3}\right) ;{ }^{13} \mathrm{C} \mathrm{NMR}\left(\mathrm{CD}_{3} \mathrm{OD}, 100\right.$ MHz): $\delta 187.7,140.1,137.1,130.4,129.6,126.6,21.3,20.1$; HRMS (EI) Calcd for $\mathrm{C}_{9} \mathrm{H}_{12} \mathrm{~N}$ : 134.0970. Found: 134.0964. 
<smiles></smiles>

1-(3-Methoxyphenyl)ethaniminium chloride (3l): Yield: $96 \% .{ }^{1} \mathrm{H}$ NMR $\left(\mathrm{CD}_{3} \mathrm{OD}, 400 \mathrm{MHz}\right) \delta$ 7.64-7.56 (m, 3H, Ar-H), 7.41-7.38 (m, 1H, Ar-H), $3.90\left(\mathrm{~s}, 3 \mathrm{H}, \mathrm{OCH}_{3}\right), 2.90\left(\mathrm{~s}, 3 \mathrm{H}, \mathrm{CH}_{3}\right) ;{ }^{13} \mathrm{C}$ NMR $\left(\mathrm{CD}_{3} \mathrm{OD}, 100 \mathrm{MHz}\right): \delta 187.5,160.6,131.6,130.8,122.5,121.9,113.5,55.4,21.4$; HRMS (EI) Calcd for $\mathrm{C}_{9} \mathrm{H}_{12} \mathrm{NO}: 150.0919$. Found: 150.0913.<smiles>CC(=[NH2+])c1cccc(Cl)c1</smiles>

1-(3-Chlorophenyl)ethaniminium chloride (3m): Yield: 80\%. ${ }^{1} \mathrm{H}$ NMR (DMSO- $d_{6}, 400 \mathrm{MHz}$ ) $\delta 12.63$ (bs, 2H, $\mathrm{NH}_{2}$ ), 8.20-8.18 (m, 1H, Ar-H), 8.05-8.02 (m, 1H, Ar-H), 7.88-7.85 (m, 2H, Ar-H), $2.85\left(\mathrm{~s}, 3 \mathrm{H}, \mathrm{CH}_{3}\right)$.<smiles>CC(=[NH2+])c1cccc(Br)c1</smiles>

1-(3-Bromophenyl)ethaniminium chloride (3n): Yield: 72\%. ${ }^{1} \mathrm{H}$ NMR (DMSO- $\left.d_{6}, 400 \mathrm{MHz}\right)$ $\delta 12.65$ (bs, 2H, $\left.\mathrm{NH}_{2}\right), 8.12-8.07(\mathrm{~m}, 1 \mathrm{H}, \mathrm{Ar}-\mathrm{H}), 7.76-7.74(\mathrm{~m}, 1 \mathrm{H}, \mathrm{Ar}-\mathrm{H}), 7.54-7.47$ (m, 2H, Ar-H), $2.88\left(\mathrm{~s}, 3 \mathrm{H}, \mathrm{CH}_{3}\right)$.<smiles>CC(=[NH2+])c1ccccc1C</smiles>

1-o-Tolylethaniminium chloride (3o): Yield: 82\%. ${ }^{1} \mathrm{H}$ NMR (DMSO- $\left.d_{6}, 500 \mathrm{MHz}\right) \delta 12.85$ (bs, $\left.2 \mathrm{H}, \mathrm{NH}_{2}\right), 7.59$ (d, $\left.1 \mathrm{H}, J=8.0 \mathrm{~Hz}, \mathrm{Ar}-\mathrm{H}\right), 7.56-7.52$ (m, 1H, Ar-H), 7.42-7.38 (m, 2H, Ar-H), $2.81\left(\mathrm{~s}, 3 \mathrm{H}, \mathrm{CH}_{3}\right), 2.41\left(\mathrm{~s}, 3 \mathrm{H}, \mathrm{CH}_{3}\right) ;{ }^{13} \mathrm{C} \mathrm{NMR}\left(\mathrm{CD}_{3} \mathrm{OD}, 100 \mathrm{MHz}\right): \delta 193.3,135.7,135.6$, 133.1, 131.9, 127.8, 126.6, 24.7, 18.7; HRMS (EI) Calcd for $\mathrm{C}_{9} \mathrm{H}_{12} \mathrm{~N}$ : 134.0970. Found: 134.0964.<smiles></smiles>

1-(2-Methoxyphenyl)ethaniminium chloride (3p): Yield: $84 \% .{ }^{1} \mathrm{H}$ NMR (DMSO- $d_{6}, 500 \mathrm{MHz}$ ) $\delta 12.54$ (bs, 2H, $\mathrm{NH}_{2}$ ), 7.93-7.91 (m, 1H, Ar-H), 7.81-7.78 (m, 1H, Ar-H), 7.34 (d, 1H, J = 8.5 $\mathrm{Hz}, \mathrm{Ar}-\mathrm{H}), 7.21-7.18(\mathrm{~m}, 1 \mathrm{H}, \mathrm{Ar}-\mathrm{H}), 3.99\left(\mathrm{~s}, 3 \mathrm{H}, \mathrm{OCH}_{3}\right), 2.82\left(\mathrm{~s}, 3 \mathrm{H}, \mathrm{CH}_{3}\right) ;{ }^{13} \mathrm{C} \mathrm{NMR}\left(\mathrm{CD}_{3} \mathrm{OD}\right.$, $100 \mathrm{MHz}$ ): $\delta 183.1,161.1,138.9,133.5,121.6,117.2,112.9,56.2,22.0$; HRMS (EI) Calcd for $\mathrm{C}_{9} \mathrm{H}_{12} \mathrm{NO}: 150.0919$. Found: 150.0913. 
<smiles>CC(=[NH2+])c1ccccc1Cl</smiles>

1-(2-Chlorophenyl)ethaniminium chloride (3q): Yield: 65\%. ${ }^{1} \mathrm{H}$ NMR (DMSO- $\left.d_{6}, 400 \mathrm{MHz}\right)$ $\delta 12.94$ (bs, 2H, NH ), 7.72-7.63 (m, 3H, Ar-H), 7.59-7.55 (m, 1H, Ar-H), 2.80 (s, 3H, $\left.\mathrm{CH}_{3}\right)$.<smiles>CC(=[NH2+])c1cccc2ccccc12</smiles>

1-(Naphthalen-1-yl)ethaniminium chloride (3r): Yield: 98\%. ${ }^{1} \mathrm{H}$ NMR (DMSO- $\left.d_{6}, 400 \mathrm{MHz}\right)$ $\delta 12.98$ (bs, 2H, $\left.\mathrm{NH}_{2}\right), 8.24$ (d, $\left.1 \mathrm{H}, J=8.4 \mathrm{~Hz}, \mathrm{Ar}-\mathrm{H}\right), 8.11-8.09$ (m, 1H, Ar-H), 8.03-8.01 (m, 1H, Ar-H), 7.90-7.88 (m, 1H, Ar-H), 7.73-7.65 (m, 3H, Ar-H), $2.97\left(\mathrm{~s}, 3 \mathrm{H}, \mathrm{CH}_{3}\right) ;{ }^{13} \mathrm{C} \mathrm{NMR}$ (DMSO- $d_{6}, 100 \mathrm{MHz}$ ): $\delta 190.3,133.7,131.6,129.5,128.9,128.7,128.6,128.0,127.8,125.7$, 124.9, 26.2; HRMS (EI) Calcd for $\mathrm{C}_{12} \mathrm{H}_{12} \mathrm{~N}$ : 170.0970. Found: 170.0964.<smiles>CC(=[NH2+])c1ccc2ccccc2c1</smiles>

1-(Naphthalen-2-yl)ethaniminium chloride (3s): Yield: 99\%. ${ }^{1} \mathrm{H}$ NMR (DMSO- $\left.d_{6}, 400 \mathrm{MHz}\right)$ $\delta 12.58\left(\mathrm{bs}, 2 \mathrm{H}, \mathrm{NH}_{2}\right), 8.85(\mathrm{~s}, 1 \mathrm{H}, \mathrm{Ar}-\mathrm{H}), 8.17-8.13(\mathrm{~m}, 3 \mathrm{H}, \mathrm{Ar}-\mathrm{H}), 8.06(\mathrm{~d}, 1 \mathrm{H}, J=8.0 \mathrm{~Hz}$, Ar-H), 7.79-7.75 (m, 1H, Ar-H), 7.72-7.68 (m, 1H, Ar-H), $2.92\left(\mathrm{~s}, 3 \mathrm{H}, \mathrm{CH}_{3}\right) ;{ }^{13} \mathrm{C} \mathrm{NMR}$ $\left(\mathrm{CD}_{3} \mathrm{OD}, 100 \mathrm{MHz}\right): \delta 186.6,136.8,133.5,132.5,130.6,130.2,129.5,127.9,127.2,122.6,21.2$; HRMS (EI) Calcd for $\mathrm{C}_{12} \mathrm{H}_{12} \mathrm{~N}$ : 170.0970. Found: 170.0964 .<smiles>CC(=[NH2+])C(C)(C)C</smiles>

3,3-Dimethylbutan-2-iminium chloride (3t): Yield: 52\%. ${ }^{1} \mathrm{H}$ NMR (DMSO- $\left.d_{6}, 400 \mathrm{MHz}\right) \delta$ 12.14 (bs, $2 \mathrm{H}, \mathrm{NH}_{2}$ ), 2.44 (s, 3H, $\mathrm{CH}_{3}$ ), $1.22\left(\mathrm{~s}, 9 \mathrm{H}, \mathrm{CH}_{3}\right) ;{ }^{13} \mathrm{C} \mathrm{NMR}$ (DMSO-d, $100 \mathrm{MHz}$ ): $\delta$ 203.1, 43.6, 26.6, 26.0, 24.7, 20.4; HRMS (EI) Calcd for $\mathrm{C}_{6} \mathrm{H}_{14} \mathrm{~N}$ : 100.1126. Found: 100.1120 .<smiles>CC(=[NH2+])C1CCCCC1</smiles>

1-Cyclohexylethaniminium chloride (3u): Yield: 71\%. ${ }^{1} \mathrm{H}$ NMR (DMSO- $\left.d_{6}, 400 \mathrm{MHz}\right) \delta$ 12.95 (bs, $\left.1 \mathrm{H}, \mathrm{NH}_{2}\right), 12.36$ (bs, $\left.1 \mathrm{H}, \mathrm{NH}_{2}\right), 2.72-2.67(\mathrm{~m}, 1 \mathrm{H}, \mathrm{CH}), 2.43\left(\mathrm{~s}, 3 \mathrm{H}, \mathrm{CH}_{3}\right)$, 1.80-1.74 (m, 4H, $\left.\mathrm{CH}_{2}\right), 1.65-1.63\left(\mathrm{~m}, 1 \mathrm{H}, \mathrm{CH}_{2}\right), 1.43-1.34\left(\mathrm{~m}, 2 \mathrm{H}, \mathrm{CH}_{2}\right), 1.27-1.14(\mathrm{~m}$, $\left.3 \mathrm{H}, \mathrm{CH}_{3}\right) ;{ }^{13} \mathrm{C} \mathrm{NMR}\left(\mathrm{CD}_{3} \mathrm{OD}, 100 \mathrm{MHz}\right): \delta 199.9,45.3,28.5,24.8,24.7,20.9$; HRMS (EI) Calcd for $\mathrm{C}_{8} \mathrm{H}_{16} \mathrm{~N}$ : 126.1277 . Found: 126.1271. 
<smiles>Cc1ccc(C(=[NH2+])c2ccccc2)cc1</smiles>

Phenyl(p-tolyl)methaniminium chloride (3v): Yield: 95\%. ${ }^{1} \mathrm{H}$ NMR (DMSO- $\left.d_{6}, 400 \mathrm{MHz}\right) \delta$ 12.58 (bs, 2H, $\left.\mathrm{NH}_{2}\right), 7.87-7.83(\mathrm{~m}, 1 \mathrm{H}, \mathrm{Ar}-\mathrm{H}), 7.76-7.74(\mathrm{~m}, 2 \mathrm{H}, \mathrm{Ar}-\mathrm{H}), 7.70-7.63$ (m, 4H, $\mathrm{Ar}-\mathrm{H}), 7.50(\mathrm{~d}, 1 \mathrm{H}, J=8.0 \mathrm{~Hz}, \mathrm{Ar}-\mathrm{H}), 2.46\left(\mathrm{~s}, 3 \mathrm{H}, \mathrm{CH}_{3}\right)$.

(B) General Procedure for Asymmetric Hydrogenation of $\mathbf{N}-\mathbf{H}$ imines Hydrochloride salts: A $5.0 \mathrm{~mL}$ vial was loaded with $[\operatorname{Ir}(\mathrm{COD}) \mathrm{Cl}]_{2}(2.1 \mathrm{mg}, 0.003 \mathrm{mmol})$ and $(S, S)$-f-Binaphane $(5.1$ $\mathrm{mg}, 0.006 \mathrm{mmol})$. The mixture was dissolved in $\mathrm{CH}_{2} \mathrm{Cl}_{2}(1 \mathrm{~mL})$ and stirred for $20 \mathrm{~min}$ at $\mathrm{rt}$ in the glovebox. To this solution was added the $\mathrm{NH}$ imine $\mathrm{HCl}$ salt in $\mathrm{MeOH}(2 \mathrm{~mL})$. The vial was then placed into a steel autoclave. The inert atmosphere was replaced by $\mathrm{H}_{2}$ and the reaction mixture was stirred under $10 \mathrm{~atm} \mathrm{H}_{2}$ (150 psi) at $\mathrm{rt}$ for $12 \mathrm{~h}$. The resulting mixture was concentrated under vacuum and dissolved in saturated aqueous $\mathrm{NaHCO}_{3}(5 \mathrm{~mL})$. After stirring for $10 \mathrm{~min}$, the mixture was extracted with $\mathrm{CH}_{2} \mathrm{Cl}_{2}(3 \times 2 \mathrm{~mL})$ and dried over $\mathrm{Na}_{2} \mathrm{SO}_{4}$. To the resulting solution was added $\mathrm{Ac}_{2} \mathrm{O}(300 \mu \mathrm{L})$ and stirred for $30 \mathrm{~min}$. The resulting solution was then analyzed for conversion and enantiomeric excess directly by GC. The product was purified by chromatography on silica gel column with dichloromethane/methanol (85:15).

\section{(C) Analytical Data for the Hydrogenation Products}

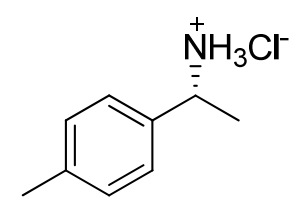

(R)-1-p-Tolylethanaminium chloride $(4 a)^{3}:[\alpha]_{\mathrm{D}}{ }^{20}+6.2\left(c 0.5, \mathrm{CH}_{2} \mathrm{Cl}_{2}\right), 95 \%$ ee, GC condition for corresponding acetamide: Supelco beta Dex ${ }^{\mathrm{TM}} 390$ column $(30 \mathrm{~m} \times 0.25 \mathrm{~mm} \times 0.25 \mu \mathrm{m}), \mathrm{He}$ $1.0 \mathrm{~mL} / \mathrm{min}$, column temperature: $140{ }^{\circ} \mathrm{C} ; t_{\mathrm{R}}=57.5 \mathrm{~min}(R), t_{\mathrm{R}}=59.7 \mathrm{~min}(S) .{ }^{1} \mathrm{H} \mathrm{NMR}\left(\mathrm{CDCl}_{3}\right.$, $400 \mathrm{MHz}) \delta 8.66\left(\mathrm{bs}, 3 \mathrm{H}, \mathrm{NH}_{3}\right), 7.34$ (d, 2H, J=8.0 Hz, Ar-H), 7.14 (d, 2H, J=8.0 Hz, Ar-H), $4.32(\mathrm{dd}, 1 \mathrm{H}, J=6.4$ and $13.2 \mathrm{~Hz}, \mathrm{CH}), 2.32\left(\mathrm{~s}, 3 \mathrm{H}, \mathrm{CH}_{3}\right), 1.63\left(\mathrm{~d}, 3 \mathrm{H}, J=6.8 \mathrm{~Hz}, \mathrm{CH}_{3}\right)$.<smiles>CC([NH3+])c1ccccc1</smiles>

(R)-1-Phenylethanaminium chloride $(\mathbf{4 b})^{4}:[\alpha]_{\mathrm{D}}^{20}+13.4\left(c \quad 0.5, \mathrm{CH}_{2} \mathrm{Cl}_{2}\right), 93 \%$ ee; GC condition for corresponding acetamide: Supelco beta $\operatorname{Dex}^{\mathrm{TM}} 390$ column $(30 \mathrm{~m} \times 0.25 \mathrm{~mm} \times$ $0.25 \mu \mathrm{m})$, He $1.0 \mathrm{~mL} / \mathrm{min}$, column temperature: $140{ }^{\circ} \mathrm{C} ; t_{\mathrm{R}}=35.2 \min (R), t_{\mathrm{R}}=36.9 \min (S) .{ }^{1} \mathrm{H}$ NMR $\left(\mathrm{CDCl}_{3}, 500 \mathrm{MHz}\right) \delta 7.98\left(\mathrm{bs}, 3 \mathrm{H}, \mathrm{NH}_{3}\right), 7.48-7.45(\mathrm{~m}, 2 \mathrm{H}, \mathrm{Ar}-\mathrm{H}), 7.37-7.30(\mathrm{~m}, 3 \mathrm{H}$, Ar-H), 4.42 (dd, $1 \mathrm{H}, J=6.0$ and $13.5 \mathrm{~Hz}, \mathrm{CH}), 1.64$ (d, $\left.3 \mathrm{H}, J=7.0 \mathrm{~Hz}, \mathrm{CH}_{3}\right)$.<smiles>CCC([NH3+])c1ccccc1</smiles>

(R)-1-Phenylpropan-1-aminium chloride (4c): ${ }^{7}[\alpha]_{\mathrm{D}}{ }^{20}+7.6$ (c $\left.0.5, \mathrm{CH}_{2} \mathrm{Cl}_{2}\right), 86 \%$ ee; GC 
condition for corresponding acetamide: Supelco beta $\operatorname{Dex}^{\mathrm{TM}} 390$ column $(30 \mathrm{~m} \times 0.25 \mathrm{~mm} \times$ $0.25 \mu \mathrm{m})$, He $1.0 \mathrm{~mL} / \mathrm{min}$, column temperature: $140{ }^{\circ} \mathrm{C}$; $t_{\mathrm{R}}=43.8 \mathrm{~min}$ (minor), $t_{\mathrm{R}}=45.7 \mathrm{~min}$ (major). ${ }^{1} \mathrm{H}$ NMR $\left(\mathrm{CDCl}_{3}, 500 \mathrm{MHz}\right) \delta 8.73\left(\mathrm{bs}, 3 \mathrm{H}, \mathrm{NH}_{3}\right), 7.44-7.41(\mathrm{~m}, 2 \mathrm{H}, \mathrm{Ar}-\mathrm{H}), 7.35-7.33$ $(\mathrm{m}, 3 \mathrm{H}, \mathrm{Ar}-\mathrm{H}), 4.06(\mathrm{dd}, 1 \mathrm{H}, J=5.5$ and $9.0 \mathrm{~Hz}, \mathrm{CH}), 2.14-2.08\left(\mathrm{~m}, 1 \mathrm{H}, \mathrm{CH}_{2}\right), 2.01-1.93(\mathrm{~m}$, $\left.1 \mathrm{H}, \mathrm{CH}_{2}\right), 0.83\left(\mathrm{t}, 3 \mathrm{H}, J=7.0 \mathrm{~Hz}, \mathrm{CH}_{3}\right)$.<smiles>CCCCC([NH3+])c1ccccc1</smiles>

(R)-1-Phenylpentan-1-aminium chloride (4d): ${ }^{4}[\alpha]_{\mathrm{D}}{ }^{20}+7.2\left(\right.$ c $\left.0.5, \mathrm{CH}_{2} \mathrm{Cl}_{2}\right), 88 \%$ ee; GC condition for corresponding acetamide: Supelco gama $\operatorname{Dex}^{\mathrm{TM}} 225$ column $(30 \mathrm{~m} \times 0.25 \mathrm{~mm} \times$ $0.25 \mu \mathrm{m}), \mathrm{He} 1.0 \mathrm{~mL} / \mathrm{min}$, programmed from $100{ }^{\circ} \mathrm{C}$ to $150{ }^{\circ} \mathrm{C}$ at $1.0{ }^{\circ} \mathrm{C} / \mathrm{min}$, hold 60 minutes; $t_{\mathrm{R}}$ $=87.8 \mathrm{~min}$ (major), $t_{\mathrm{R}}=89.1 \mathrm{~min}$ (minor). ${ }^{1} \mathrm{H} \mathrm{NMR}\left(\mathrm{CDCl}_{3}, 500 \mathrm{MHz}\right) \delta 8.70\left(\mathrm{bs}, 3 \mathrm{H}, \mathrm{NH}_{3}\right)$, 7.43-7.41 (m, 2H, Ar-H), 7.35-7.33 (m, 3H, Ar-H), $4.11(\mathrm{dd}, 1 \mathrm{H}, J=5.5$ and $9.5 \mathrm{~Hz}, \mathrm{CH})$, 2.10-2.03 (m, 1H, $\left.\mathrm{CH}_{2}\right), 1.97-1.89\left(\mathrm{~m}, 1 \mathrm{H}, \mathrm{CH}_{2}\right), 1.33-1.18\left(\mathrm{~m}, 3 \mathrm{H}, \mathrm{CH}_{2}\right), 1.13-1.05(\mathrm{~m}, 1 \mathrm{H}$, $\left.\mathrm{CH}_{2}\right), 0.82\left(\mathrm{t}, 3 \mathrm{H}, J=7.0 \mathrm{~Hz}, \mathrm{CH}_{3}\right)$.<smiles>CC(C)(C)[C@@H]([NH3+])c1ccccc1</smiles>

(R)-2,2-Dimethyl-1-phenylpropan-1-aminium chloride (4e): ${ }^{4}[\alpha]_{\mathrm{D}}{ }^{20}+7.0$ (c $0.5, \mathrm{CH}_{2} \mathrm{Cl}_{2}$ ), $80 \%$ ee, GC condition for corresponding acetamide: Supelco gama $\operatorname{Dex}^{\mathrm{TM}} 225$ column $(30 \mathrm{~m} \times$ $0.25 \mathrm{~mm} \times 0.25 \mu \mathrm{m}), \mathrm{He} 1.0 \mathrm{~mL} / \mathrm{min}$, column temperature: $160{ }^{\circ} \mathrm{C} ; t_{\mathrm{R}}=20.8 \mathrm{~min}$ (minor), $t_{\mathrm{R}}=$ $21.3 \mathrm{~min}$ (major). ${ }^{1} \mathrm{H}$ NMR $\left(\mathrm{CDCl}_{3}, 500 \mathrm{MHz}\right) \delta 8.80\left(\mathrm{bs}, 3 \mathrm{H}, \mathrm{NH}_{3}\right), 7.70-7.67(\mathrm{~m}, 2 \mathrm{H}, \mathrm{Ar}-\mathrm{H})$, 7.42-7.38 (m, 3H, Ar-H), $4.02(\mathrm{dd}, 1 \mathrm{H}, J=5.5$ and $9.0 \mathrm{~Hz}, \mathrm{CH}), 1.02\left(\mathrm{~s}, 9 \mathrm{H}, \mathrm{CH}_{3}\right)$.<smiles>COc1ccc(C(C)[NH3+])cc1</smiles>

(R)-1-(4-methoxyphenyl)ethanaminium chloride (4f): ${ }^{8}[\alpha]_{\mathrm{D}}{ }^{20}-14.4\left(\mathrm{c} 0.5, \mathrm{CH}_{2} \mathrm{Cl}_{2}\right), 93 \%$ ee,; GC condition for corresponding acetamide: Supelco gama $\operatorname{Dex}^{\mathrm{TM}} 225$ column $(30 \mathrm{~m} \times 0.25 \mathrm{~mm}$ $\times 0.25 \mu \mathrm{m}$ ), He $1.0 \mathrm{~mL} / \mathrm{min}$, column temperature: $160{ }^{\circ} \mathrm{C} ; t_{\mathrm{R}}=55.5 \mathrm{~min}$ (minor), $t_{\mathrm{R}}=58.1 \mathrm{~min}$ (major). ${ }^{1} \mathrm{H} \mathrm{NMR}\left(\mathrm{CDCl}_{3}, 400 \mathrm{MHz}\right) \delta 8.11\left(\mathrm{bs}, 3 \mathrm{H}, \mathrm{NH}_{3}\right), 7.38(\mathrm{~d}, 2 \mathrm{H}, J=8.8 \mathrm{~Hz}, \mathrm{Ar}-\mathrm{H}), 7.14$ $(\mathrm{d}, 2 \mathrm{H}, J=8.8 \mathrm{~Hz}, \mathrm{Ar}-\mathrm{H}), 4.29(\mathrm{dd}, 1 \mathrm{H}, J=6.4$ and $13.2 \mathrm{~Hz}, \mathrm{CH}), 3.77\left(\mathrm{~s}, 3 \mathrm{H}, \mathrm{OCH}_{3}\right), 1.61(\mathrm{~d}$, $\left.3 \mathrm{H}, J=6.8 \mathrm{~Hz}, \mathrm{CH}_{3}\right)$.<smiles>C[C@H]([NH3+])c1ccc(F)cc1</smiles>

(R)-1-(4-Fluorophenyl)ethanaminium chloride (4g): ${ }^{9}[\alpha]_{\mathrm{D}}{ }^{20}+13.4$ (c $\left.0.5, \mathrm{CH}_{2} \mathrm{Cl}_{2}\right), 92 \%$ ee,; GC condition for corresponding acetamide: Supelco beta Dex ${ }^{\mathrm{TM}} 390$ column $(30 \mathrm{~m} \times 0.25 \mathrm{~mm} \times$ $0.25 \mu \mathrm{m})$, He $1.0 \mathrm{~mL} / \mathrm{min}$, programmed from $100{ }^{\circ} \mathrm{C}$ to $180{ }^{\circ} \mathrm{C}$ at $1.0{ }^{\circ} \mathrm{C} / \mathrm{min} ; t_{\mathrm{R}}=56.8 \mathrm{~min}$ (minor), $t_{\mathrm{R}}=57.7 \mathrm{~min}$ (major). ${ }^{1} \mathrm{H} \mathrm{NMR}\left(\mathrm{CDCl}_{3}, 500 \mathrm{MHz}\right) \delta 8.12$ (bs, $\left.3 \mathrm{H}, \mathrm{NH}_{3}\right), 7.47-7.43(\mathrm{~m}$, 2H, Ar-H), 7.06-7.02 (m, 2H, Ar-H), 4.34 (dd, 1H, J = 8.5 and 13.0 Hz, CH), 1.62 (d, 3H, $J=$ 
$\left.6.8 \mathrm{~Hz}, \mathrm{CH}_{3}\right)$.<smiles>CC([NH3+])c1ccc(Cl)cc1</smiles>

(+)-1-(4-chlorophenyl)ethanaminium chloride (4h): $[\alpha]_{\mathrm{D}}{ }^{20}+12.8\left(\right.$ c $\left.0.5, \mathrm{CH}_{2} \mathrm{Cl}_{2}\right)$, 94\% ee, $\mathrm{GC}$ condition for corresponding acetamide: Supelco gama Dex ${ }^{\mathrm{TM}} 225$ column $(30 \mathrm{~m} \times 0.25 \mathrm{~mm}$ $\times 0.25 \mu \mathrm{m}$ ), He $1.0 \mathrm{~mL} / \mathrm{min}$, column temperature: $160{ }^{\circ} \mathrm{C} ; t_{\mathrm{R}}=59.3 \mathrm{~min}$ (minor), $t_{\mathrm{R}}=61.9 \mathrm{~min}$ (major). ${ }^{1} \mathrm{H} \mathrm{NMR}\left(\mathrm{CDCl}_{3}, 500 \mathrm{MHz}\right) \delta 7.39$ (d, 2H, $\left.J=8.5 \mathrm{~Hz}, \mathrm{Ar}-\mathrm{H}\right), 7.33$ (d, $2 \mathrm{H}, J=8.5 \mathrm{~Hz}$, Ar-H), 4.29 (dd, $1 \mathrm{H}, J=8.5$ and $13.0 \mathrm{~Hz}, \mathrm{CH}), 1.59$ (d, $\left.3 \mathrm{H}, J=6.5 \mathrm{~Hz}, \mathrm{CH}_{3}\right)$.<smiles>CC([NH3+])c1ccc(Br)cc1</smiles>

(R)-1-(4-Bromophenyl)ethanaminium chloride (4i): ${ }^{8}[\alpha]_{\mathrm{D}}{ }^{20}+15.2\left(\mathrm{c} 0.5, \mathrm{CH}_{2} \mathrm{Cl}_{2}\right), 93 \%$ ee; GC condition for corresponding acetamide: Supelco beta Dex ${ }^{\mathrm{TM}} 390$ column $(30 \mathrm{~m} \times 0.25 \mathrm{~mm} \times$ $0.25 \mu \mathrm{m}$ ), He $1.0 \mathrm{~mL} / \mathrm{min}$, column temperature: $150{ }^{\circ} \mathrm{C}$; $t_{\mathrm{R}}=130.8 \mathrm{~min}$ (minor), $t_{\mathrm{R}}=136.0 \mathrm{~min}$ (major). ${ }^{1} \mathrm{H}$ NMR $\left(\mathrm{CDCl}_{3}, 500 \mathrm{MHz}\right) \delta 7.46(\mathrm{~d}, 2 \mathrm{H}, J=8.5 \mathrm{~Hz}, \mathrm{Ar}-\mathrm{H}), 7.26(\mathrm{~d}, 2 \mathrm{H}, J=8.5 \mathrm{~Hz}$, Ar-H), 4.17 (dd, $1 \mathrm{H}, J=8.5$ and $13.0 \mathrm{~Hz}, \mathrm{CH}), 1.44\left(\mathrm{~d}, 3 \mathrm{H}, J=6.5 \mathrm{~Hz}, \mathrm{CH}_{3}\right)$.<smiles>CC([NH3+])c1ccc(C(F)(F)F)cc1</smiles>

(R)-1-(4-(Trifluoromethyl)phenyl)ethanaminium chloride $(4 \mathbf{j}):^{8}[\alpha]_{\mathrm{D}}{ }^{20}+5.4\left(c \quad 0.5, \mathrm{CH}_{2} \mathrm{Cl}_{2}\right)$, 93\% ee, GC condition for corresponding acetamide: Supelco gama $\operatorname{Dex}^{\mathrm{TM}} 225$ column $(30 \mathrm{~m} \times$ $0.25 \mathrm{~mm} \times 0.25 \mu \mathrm{m}), \mathrm{He} 1.0 \mathrm{~mL} / \mathrm{min}$, column temperature: $150{ }^{\circ} \mathrm{C} ; t_{\mathrm{R}}=43.0 \mathrm{~min}$ (minor), $t_{\mathrm{R}}=$ $46.1 \mathrm{~min}$ (major). ${ }^{1} \mathrm{H} \mathrm{NMR}\left(\mathrm{CDCl}_{3}, 400 \mathrm{MHz}\right) \delta 8.21$ (bs, 3H, $\left.\mathrm{NH}_{3}\right), 7.52-7.50(\mathrm{~m}, 2 \mathrm{H}, \mathrm{Ar}-\mathrm{H})$, 7.49-7.48 (m, 2H, Ar-H), $4.40(\mathrm{dd}, 1 \mathrm{H}, J=8.5$ and $13.0 \mathrm{~Hz}, \mathrm{CH}), 1.63\left(\mathrm{~d}, 3 \mathrm{H}, J=6.8 \mathrm{~Hz}, \mathrm{CH}_{3}\right)$.<smiles></smiles>

(+)-1-m-Tolylethanaminium chloride (4k): $[\alpha]_{\mathrm{D}}{ }^{20}+12.4\left(\right.$ c $\left.0.5, \mathrm{CH}_{2} \mathrm{Cl}_{2}\right), 92 \%$ ee; GC condition for corresponding acetamide: Supelco beta $\operatorname{Dex}^{\mathrm{TM}} 390$ column $(30 \mathrm{~m} \times 0.25 \mathrm{~mm} \times$ $0.25 \mu \mathrm{m})$, He $1.0 \mathrm{~mL} / \mathrm{min}$, programmed from $100{ }^{\circ} \mathrm{C}$ to $180{ }^{\circ} \mathrm{C}$ at $1.0{ }^{\circ} \mathrm{C} / \mathrm{min} ; t_{\mathrm{R}}=60.2 \mathrm{~min}$ (minor), $t_{\mathrm{R}}=61.2 \mathrm{~min}$ (major). ${ }^{1} \mathrm{H} \mathrm{NMR}\left(\mathrm{CDCl}_{3}, 500 \mathrm{MHz}\right) \delta 8.56\left(\mathrm{bs}, 3 \mathrm{H}, \mathrm{NH}_{3}\right), 7.29-7.22(\mathrm{~m}$, $3 \mathrm{H}, \mathrm{Ar}-\mathrm{H}), 7.13(\mathrm{~d}, 1 \mathrm{H}, J=7.0 \mathrm{~Hz}, \mathrm{Ar}-\mathrm{H}), 4.32(\mathrm{dd}, 1 \mathrm{H}, J=6.5$ and $13.5 \mathrm{~Hz}, \mathrm{CH}), 2.32(\mathrm{~s}, 3 \mathrm{H}$, $\left.\mathrm{CH}_{3}\right), 1.65\left(\mathrm{~d}, 3 \mathrm{H}, J=7.0 \mathrm{~Hz}, \mathrm{CH}_{3}\right)$.<smiles>COc1cccc(C(C)[NH3+])c1</smiles> 
(+)-1-(3-Methoxyphenyl)ethanaminium chloride (4l): $[\alpha]_{\mathrm{D}}{ }^{20}+13.6\left(\right.$ c $\left.0.5, \mathrm{CH}_{2} \mathrm{Cl}_{2}\right), 94 \%$ ee,; GC condition for corresponding acetamide: Supelco gama Dex ${ }^{\mathrm{TM}} 225$ column $(30 \mathrm{~m} \times 0.25 \mathrm{~mm}$ $\times 0.25 \mu \mathrm{m}$ ), He $1.0 \mathrm{~mL} / \mathrm{min}$, column temperature: $160{ }^{\circ} \mathrm{C} ; t_{\mathrm{R}}=45.9 \mathrm{~min}\left(\right.$ minor), $t_{\mathrm{R}}=49.1 \mathrm{~min}$ (major). ${ }^{1} \mathrm{H} \mathrm{NMR}\left(\mathrm{CDCl}_{3}, 500 \mathrm{MHz}\right) \delta 8.68\left(\mathrm{bs}, 3 \mathrm{H}, \mathrm{NH}_{3}\right), 7.24(\mathrm{t}, 1 \mathrm{H}, J=8.0 \mathrm{~Hz}, \mathrm{Ar}-\mathrm{H}), 7.09$ $(\mathrm{s}, 1 \mathrm{H}, \mathrm{Ar}-\mathrm{H}), 7.01(\mathrm{~d}, 1 \mathrm{H}, J=7.5 \mathrm{~Hz}, \mathrm{Ar}-\mathrm{H}), 6.85(\mathrm{dd}, 1 \mathrm{H}, J=2.0$ and $7.0 \mathrm{~Hz}), 4.34(\mathrm{dd}, 1 \mathrm{H}, J=$ 6.5 and $13.5 \mathrm{~Hz}, \mathrm{CH}), 3.73\left(\mathrm{~s}, 3 \mathrm{H}, \mathrm{OCH}_{3}\right), 1.66\left(\mathrm{~d}, 3 \mathrm{H}, J=7.0 \mathrm{~Hz}, \mathrm{CH}_{3}\right)$.<smiles>C[C@H]([NH3+])c1cccc(Cl)c1</smiles>

(+)-1-(3-Chlorophenyl)ethanaminium chloride (4m): $[\alpha]_{\mathrm{D}}{ }^{20}+2.1\left(\right.$ c $\left.0.5, \mathrm{CH}_{2} \mathrm{Cl}_{2}\right), 92 \%$ ee, GC condition for corresponding acetamide: Supelco gama Dex ${ }^{\mathrm{TM}} 225$ column $(30 \mathrm{~m} \times 0.25 \mathrm{~mm}$ $\times 0.25 \mu \mathrm{m}$ ), He $1.0 \mathrm{~mL} / \mathrm{min}$, column temperature: $150{ }^{\circ} \mathrm{C} ; t_{\mathrm{R}}=83.6 \mathrm{~min}$ (minor), $t_{\mathrm{R}}=98.7 \mathrm{~min}$ (major). ${ }^{1} \mathrm{H}$ NMR $\left(\mathrm{CDCl}_{3}, 400 \mathrm{MHz}\right) \delta 8.65\left(\mathrm{bs}, 3 \mathrm{H}, \mathrm{NH}_{3}\right), 7.48-7.47(\mathrm{~m}, 1 \mathrm{H}, \mathrm{Ar}-\mathrm{H}), 7.40-7.37$ (m, 1H, Ar-H), 7.31-7.29 (m, 2H, Ar-H), 4.32 (dd, 1H, $J=6.4$ and $13.2 \mathrm{~Hz}, \mathrm{CH}), 1.63$ (d, 3H, $J$ $\left.=6.4 \mathrm{~Hz}, \mathrm{CH}_{3}\right)$.<smiles>CC([NH3+])c1cccc(Br)c1</smiles>

(+)-1-(3-Bromophenyl)ethanaminium chloride (4n): $\left.[\alpha]_{\mathrm{D}}{ }^{20}+8.4(c) .5, \mathrm{CH}_{2} \mathrm{Cl}_{2}\right), 91 \%$ ee; GC condition for corresponding acetamide: Supelco beta $\operatorname{Dex}^{\mathrm{TM}} 225$ column $(30 \mathrm{~m} \times 0.25 \mathrm{~mm} \times$ $0.25 \mu \mathrm{m})$, He $1.0 \mathrm{~mL} / \mathrm{min}$, column temperature: $160{ }^{\circ} \mathrm{C} ; t_{\mathrm{R}}=71.2 \mathrm{~min}$ (minor), $t_{\mathrm{R}}=78.5 \mathrm{~min}$ (major). ${ }^{1} \mathrm{H} \mathrm{NMR}\left(\mathrm{CDCl}_{3}, 400 \mathrm{MHz}\right) \delta 8.62$ (bs, 3H, $\left.\mathrm{NH}_{3}\right), 7.60(\mathrm{~s}, 1 \mathrm{H}, \mathrm{Ar}-\mathrm{H}), 7.45-7.40$ (m, 2H, Ar-H), 7.24-7.20 (m, 1H, Ar-H), $4.29(\mathrm{dd}, 1 \mathrm{H}, J=6.4$ and $13.2 \mathrm{~Hz}, \mathrm{CH}), 1.61(\mathrm{~d}, 3 \mathrm{H}, J=6.8 \mathrm{~Hz}$, $\left.\mathrm{CH}_{3}\right)$.<smiles>Cc1ccccc1[C+](C)[N-]Cl</smiles>

(+)-1-o-Tolylethanaminium chloride (4o): $[\alpha]_{\mathrm{D}}{ }^{20}+14.6\left(c 0.5, \mathrm{CH}_{2} \mathrm{Cl}_{2}\right), 81 \%$ ee,; GC condition for corresponding acetamide: Supelco gama Dex ${ }^{\mathrm{TM}} 225$ column $(30 \mathrm{~m} \times 0.25 \mathrm{~mm} \times 0.25 \mu \mathrm{m})$, He $1.0 \mathrm{~mL} / \mathrm{min}$, column temperature: $160{ }^{\circ} \mathrm{C} ; t_{\mathrm{R}}=20.8 \mathrm{~min}$ (minor), $t_{\mathrm{R}}=21.4 \mathrm{~min}$ (major). ${ }^{1} \mathrm{H}$ NMR $\left(\mathrm{CDCl}_{3}, 500 \mathrm{MHz}\right) \delta 8.20$ (bs, 3H, NH$), 7.62-7.60(\mathrm{~m}, 1 \mathrm{H}, \mathrm{Ar}-\mathrm{H}), 7.24-7.19(\mathrm{~m}, 2 \mathrm{H}$, Ar-H), 7.17-7.15 (m, 1H, Ar-H), $4.64(\mathrm{dd}, 1 \mathrm{H}, J=6.4$ and $13.2 \mathrm{~Hz}, \mathrm{CH}), 2.37\left(\mathrm{~s}, 3 \mathrm{H}, \mathrm{CH}_{3}\right), 1.62$ (d, $3 \mathrm{H}, J=6.5 \mathrm{~Hz}, \mathrm{CH}_{3}$ ).<smiles></smiles>

(R)-1-(2-Methoxyphenyl)ethanaminium chloride (4p): ${ }^{10}[\alpha]_{\mathrm{D}}{ }^{20}-12.2\left(\right.$ c $\left.0.5, \mathrm{CH}_{2} \mathrm{Cl}_{2}\right), 92 \%$ ee,; GC condition for corresponding acetamide: Supelco beta $\operatorname{Dex}^{\mathrm{TM}} 225$ column $(30 \mathrm{~m} \times 0.25 \mathrm{~mm} \times$ 
$0.25 \mu \mathrm{m}$ ), He $1.0 \mathrm{~mL} / \mathrm{min}$, column temperature: $150{ }^{\circ} \mathrm{C} ; t_{\mathrm{R}}=44.5 \mathrm{~min}$ (minor), $t_{\mathrm{R}}=46.7 \mathrm{~min}$ (major). ${ }^{1} \mathrm{H}$ NMR $\left(\mathrm{CDCl}_{3}, 500 \mathrm{MHz}\right) \delta 8.47$ (bs, 3H, $\left.\mathrm{NH}_{3}\right), 7.41-7.39$ (m, 1H, Ar-H), 7.32-7.28 (m, 1H, Ar-H), 6.96-6.94 (m, 1H, Ar-H), 6.87-6.85 (m, 1H, Ar-H), 4.69 (dd, 1H, $J=6.5$ and $13.5 \mathrm{~Hz}, \mathrm{CH}), 3.83\left(\mathrm{~s}, 3 \mathrm{H}, \mathrm{OCH}_{3}\right), 1.69$ (d, 3H, $\left.J=7.0 \mathrm{~Hz}, \mathrm{CH}_{3}\right)$.<smiles>CC([NH3+])c1ccccc1Cl</smiles>

(+)-1-(2-Chlorophenyl)ethanaminium chloride (4q): $[\alpha]_{\mathrm{D}}{ }^{20}+15.9$ (c $\left.0.5, \mathrm{CH}_{2} \mathrm{Cl}_{2}\right)$, 81\% ee, GC condition for corresponding acetamide: Supelco gama $\operatorname{Dex}^{\mathrm{TM}} 225$ column $(30 \mathrm{~m} \times 0.25 \mathrm{~mm}$ $\times 0.25 \mu \mathrm{m}$ ), He $1.0 \mathrm{~mL} / \mathrm{min}$, column temperature: $150{ }^{\circ} \mathrm{C} ; t_{\mathrm{R}}=55.4 \mathrm{~min}(\operatorname{minor}), t_{\mathrm{R}}=64.4 \mathrm{~min}$ (major). ${ }^{1} \mathrm{H}$ NMR $\left(\mathrm{CDCl}_{3}, 500 \mathrm{MHz}\right) \delta$ 7.65-7.62 (m, 1H, Ar-H), 7.38-7.36 (m, 1H, Ar-H), 7.31-7.27 (m, 1H, Ar-H), 7.24-7.20 (m, 1H, Ar-H), $4.74(\mathrm{dd}, 1 \mathrm{H}, J=6.5$ and $13.0 \mathrm{~Hz}, \mathrm{CH}), 2.37$ $\left(\mathrm{s}, 3 \mathrm{H}, \mathrm{CH}_{3}\right), 1.55\left(\mathrm{~d}, 3 \mathrm{H}, J=6.5 \mathrm{~Hz}, \mathrm{CH}_{3}\right)$.<smiles>C[C@H]([NH3+])c1cccc2ccccc12</smiles>

(-)-1-(Naphthalen-1-yl)ethanaminium chloride (4r): ${ }^{5}[\alpha]_{\mathrm{D}}{ }^{20}-14.2$ (c $\left.0.5, \mathrm{CH}_{2} \mathrm{Cl}_{2}\right), 93 \%$ ee,; GC condition for corresponding acetamide: Supelco gama $\operatorname{Dex}^{\mathrm{TM}} 225$ column $(30 \mathrm{~m} \times 0.25 \mathrm{~mm}$ $\times 0.25 \mu \mathrm{m}$ ), He $1.0 \mathrm{~mL} / \mathrm{min}$, column temperature: $175{ }^{\circ} \mathrm{C} ; t_{\mathrm{R}}=70.1 \mathrm{~min}$ (minor), $t_{\mathrm{R}}=78.6 \mathrm{~min}$ (major). ${ }^{1} \mathrm{H}$ NMR $\left(\mathrm{CDCl}_{3}, 400 \mathrm{MHz}\right) \delta 8.02-7.99(\mathrm{~m}, 1 \mathrm{H}, \mathrm{Ar}-\mathrm{H}), 7.89-7.86(\mathrm{~m}, 1 \mathrm{H}, \mathrm{Ar}-\mathrm{H})$, 7.80-7.77 (m, 2H, Ar-H), 7.56-7.44 (m, 3H, Ar-H), 6.06 (bs, 3H, NH $), 5.18(\mathrm{dd}, 1 \mathrm{H}, J=6.4$ and $13.2 \mathrm{~Hz}, \mathrm{CH}), 1.72\left(\mathrm{~d}, 3 \mathrm{H}, J=6.4 \mathrm{~Hz}, \mathrm{CH}_{3}\right)$.<smiles>CC([NH3+])c1ccc2ccccc2c1</smiles>

(R)-1-(Naphthalen-2-yl)ethanaminium chloride (4s): ${ }^{11}[\alpha]_{\mathrm{D}}{ }^{20}+9.2\left(\right.$ ( $\left.1.0, \mathrm{CH}_{2} \mathrm{Cl}_{2}\right), 92 \%$ ee,; GC condition for corresponding acetamide: Supelco beta Dex ${ }^{\mathrm{TM}} 225$ column $(30 \mathrm{~m} \times 0.25 \mathrm{~mm} \times$ $0.25 \mu \mathrm{m})$, He $1.0 \mathrm{~mL} / \mathrm{min}$, programmed from $165{ }^{\circ} \mathrm{C}$ to $185{ }^{\circ} \mathrm{C}$ at $1.0{ }^{\circ} \mathrm{C} / \mathrm{min}$, hold 100 minutes at $185{ }^{\circ} \mathrm{C} ; t_{\mathrm{R}}=62.1 \mathrm{~min}$ (minor), $t_{\mathrm{R}}=65.5 \mathrm{~min}$ (major). ${ }^{1} \mathrm{H} \mathrm{NMR}\left(\mathrm{CDCl}_{3}, 500 \mathrm{MHz}\right) \delta 7.85(\mathrm{~s}$, 1H, Ar-H), 7.78-7.72 (m, 3H, Ar-H), 7.55-7.53 (m, 1H, Ar-H), 7.50-7.44 (m, 2H, Ar-H), 4.42 (dd, $1 \mathrm{H}, J=6.5$ and $13.5 \mathrm{~Hz}, \mathrm{CH}), 1.64\left(\mathrm{~d}, 3 \mathrm{H}, J=7.0 \mathrm{~Hz}, \mathrm{CH}_{3}\right.$ ).<smiles>CC([NH3+])C(C)(C)C</smiles>

(R)-3,3-Dimethylbutan-2-aminium chloride (4t): ${ }^{8}[\alpha]_{\mathrm{D}}{ }^{20}-2.8$ (c $\left.0.5, \mathrm{CH}_{2} \mathrm{Cl}_{2}\right), 17 \%$ ee, GC condition for corresponding acetamide: Supelco gama $\operatorname{Dex}^{\mathrm{TM}} 225$ column $(30 \mathrm{~m} \times 0.25 \mathrm{~mm} \times$ $0.25 \mu \mathrm{m}$ ), He $1.0 \mathrm{~mL} / \mathrm{min}$, column temperature: $120{ }^{\circ} \mathrm{C} ; t_{\mathrm{R}}=18.5 \mathrm{~min}$ (major), $t_{\mathrm{R}}=20.5 \mathrm{~min}$ (minor). ${ }^{1} \mathrm{H}$ NMR $\left(\mathrm{CDCl}_{3}, 500 \mathrm{MHz}\right) \delta 8.33$ (bs, $\left.3 \mathrm{H}, \mathrm{NH}_{3}\right), 3.10(\mathrm{dd}, 1 \mathrm{H}, J=7.0$ and $13.5 \mathrm{~Hz}$, $\mathrm{CH}), 1.37$ (d, $\left.3 \mathrm{H}, J=7.0 \mathrm{~Hz}, \mathrm{CH}_{3}\right), 1.07\left(\mathrm{~s}, 9 \mathrm{H}, \mathrm{CH}_{3}\right)$. 
<smiles>CC([NH3+])C1CCCCC1</smiles>

(R)-1-Cyclohexylethanaminium chloride (4u): ${ }^{6}[\alpha]_{\mathrm{D}}{ }^{20}+5.1$ (c 1.0, $\left.\mathrm{CH}_{2} \mathrm{Cl}_{2}\right), 73 \%$ ee; GC condition for corresponding acetamide: Supelco beta $\operatorname{Dex}^{\mathrm{TM}} 390$ column $(30 \mathrm{~m} \times 0.25 \mathrm{~mm} \times$ $0.25 \mu \mathrm{m}$ ), He $1.0 \mathrm{~mL} / \mathrm{min}$, column temperature: $140{ }^{\circ} \mathrm{C} ; t_{\mathrm{R}}=27.8 \mathrm{~min}$ (major), $t_{\mathrm{R}}=29.2 \mathrm{~min}$ (minor). ${ }^{1} \mathrm{H}$ NMR $\left(\mathrm{CDCl}_{3}, 400 \mathrm{MHz}\right) \delta 8.33$ (bs, 3H, NH ), 3.16-3.10 (m, 1H, CH), 1.90-1.66 $\left(\mathrm{m}, 5 \mathrm{H}, \mathrm{CH}, \mathrm{CH}_{2}\right), 1.37\left(\mathrm{~d}, 3 \mathrm{H}, J=6.8 \mathrm{~Hz}, \mathrm{CH}_{3}\right), 1.29-1.04\left(\mathrm{~m}, 6 \mathrm{H}, \mathrm{CH}_{2}\right)$.<smiles>Cc1ccc(C([NH3+])c2ccccc2)cc1</smiles>

(R)-Phenyl(p-tolyl)methanaminium chloride (4v): ${ }^{12}[\alpha]_{\mathrm{D}}{ }^{20}-6.2$ (c 1.0, $\left.\mathrm{CH}_{2} \mathrm{Cl}_{2}\right), 17 \%$ ee,; HPLC condition for corresponding acetamide: Chiralcel OD-H column, $n$-hexane/2-propanol = 90:10, $1.0 \mathrm{~mL} / \mathrm{min}, 222 \mathrm{~nm} \mathrm{UV}$ detector, $t_{\mathrm{R}}=7.7 \mathrm{~min}$ (minor), $t_{\mathrm{R}}=9.4 \mathrm{~min}$ (major). ${ }^{1} \mathrm{H} \mathrm{NMR}$ $\left(\mathrm{CDCl}_{3}, 400 \mathrm{MHz}\right) \delta 9.10\left(\mathrm{bs}, 3 \mathrm{H}, \mathrm{NH}_{2}\right), 7.36(\mathrm{~d}, 2 \mathrm{H}, J=6.4 \mathrm{~Hz}, \mathrm{Ar}-\mathrm{H}), 7.27-7.24(\mathrm{~m}, 5 \mathrm{H}$, Ar-H), 7.07 (d, 2H, J=7.6 Hz, Ar-H), 5.38 (s, 1H, CH), 2.33 (s, 3H, $\mathrm{CH}_{3}$ ).

(D) Imine Deuteration: Imine hydrochloride 3a (22 $\mathrm{mg}, 0.129 \mathrm{mmol})$ was dissolved in anhydrous $\mathrm{MeOH}(2 \mathrm{~mL})$. In a separate vessel were dissolved $[\operatorname{Ir}(\mathrm{COD}) \mathrm{Cl}]_{2}(2.1 \mathrm{mg}, 0.003 \mathrm{mmol})$ and $(S, S)$-f-binaphane $(5.1 \mathrm{mg}, 0.006 \mathrm{mmol})$ in anhydrous $\mathrm{CH}_{2} \mathrm{Cl}_{2}(1 \mathrm{~mL})$. The resulting solution was stirred for $20 \mathrm{~min}$ at $\mathrm{rt}$. The catalyst solution was added to the imine solution. The resulting solution was degassed and then pressurized with $\mathrm{D}_{2}(150 \mathrm{psi})$ with shaking for $20 \mathrm{~h}$ at $25^{\circ} \mathrm{C}$ on a Symyx ${ }^{\circledR}$ HPR/HOSS system. After venting, the solution was concentrated under vacuum and dissolved in $\mathrm{CDCl}_{3}$ for $500 \mathrm{MHz}{ }^{1} \mathrm{H}$ NMR spectroscopic analysis which showed exclusive formation of deuterio- $4 a$ ( $\sim 60 \% \mathrm{D}$-incorporation).

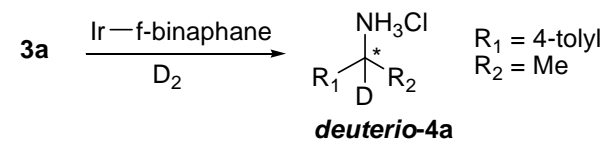




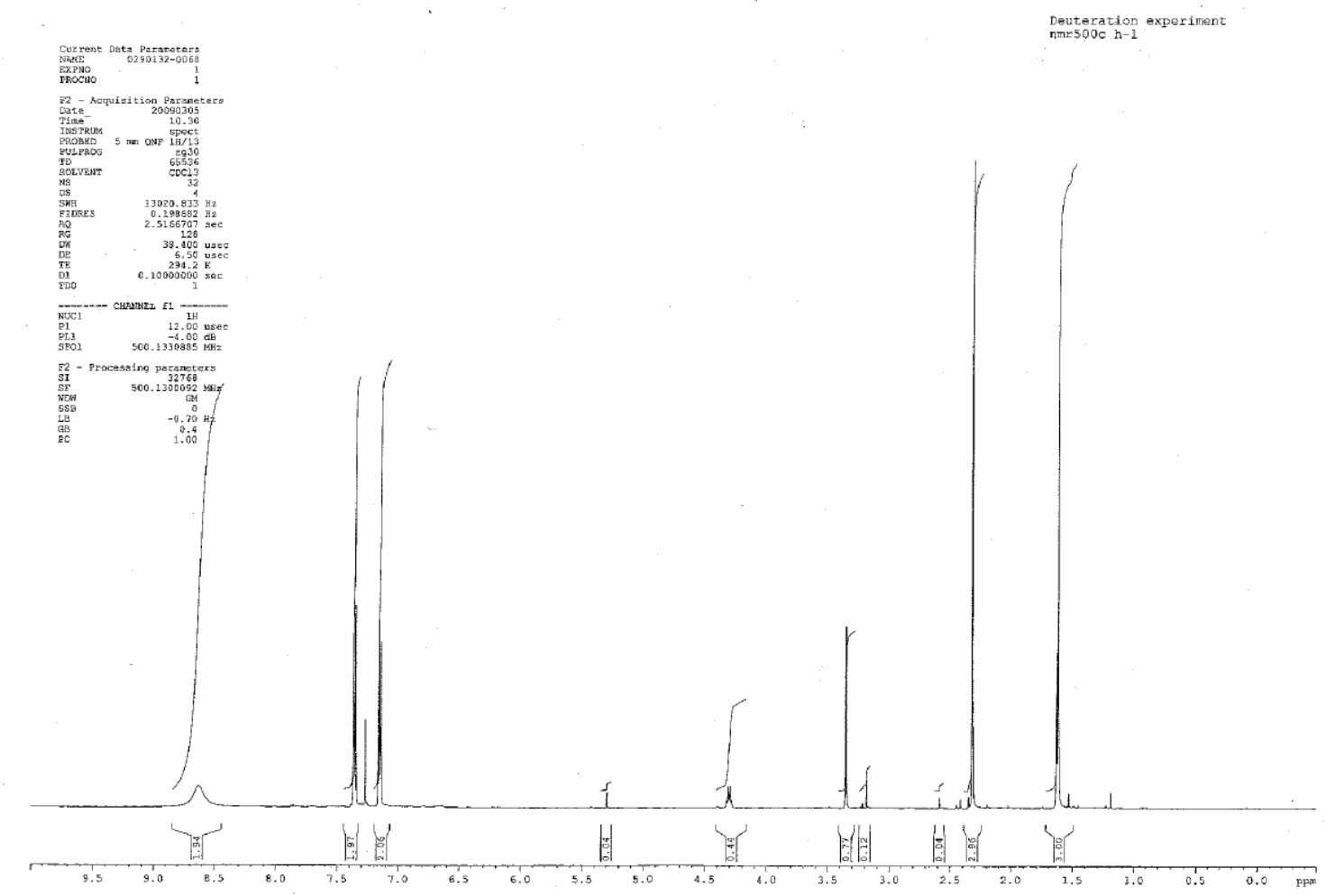

(E) NMR Spectra of New Products

1-p-Tolylethaniminium chloride (3a)

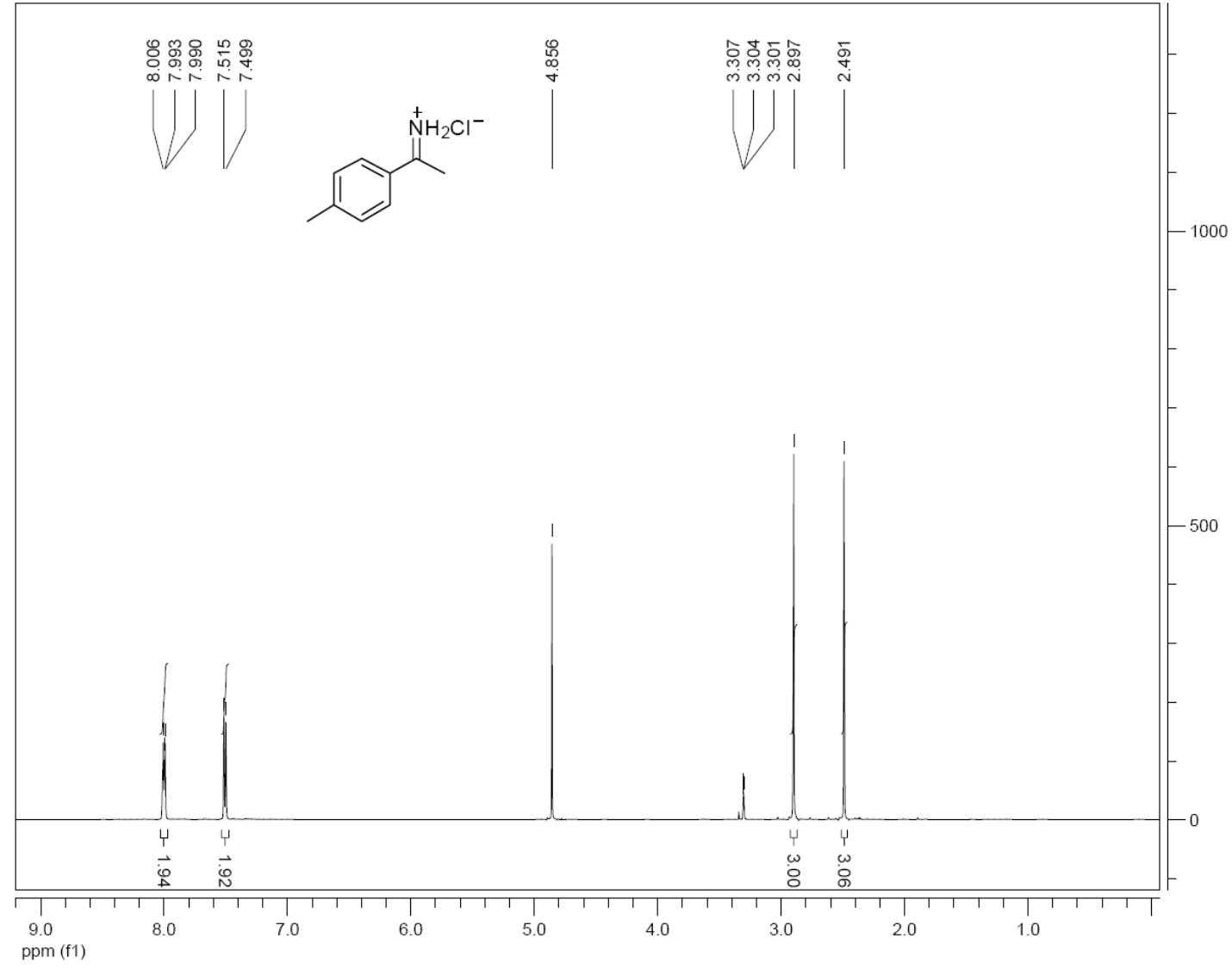




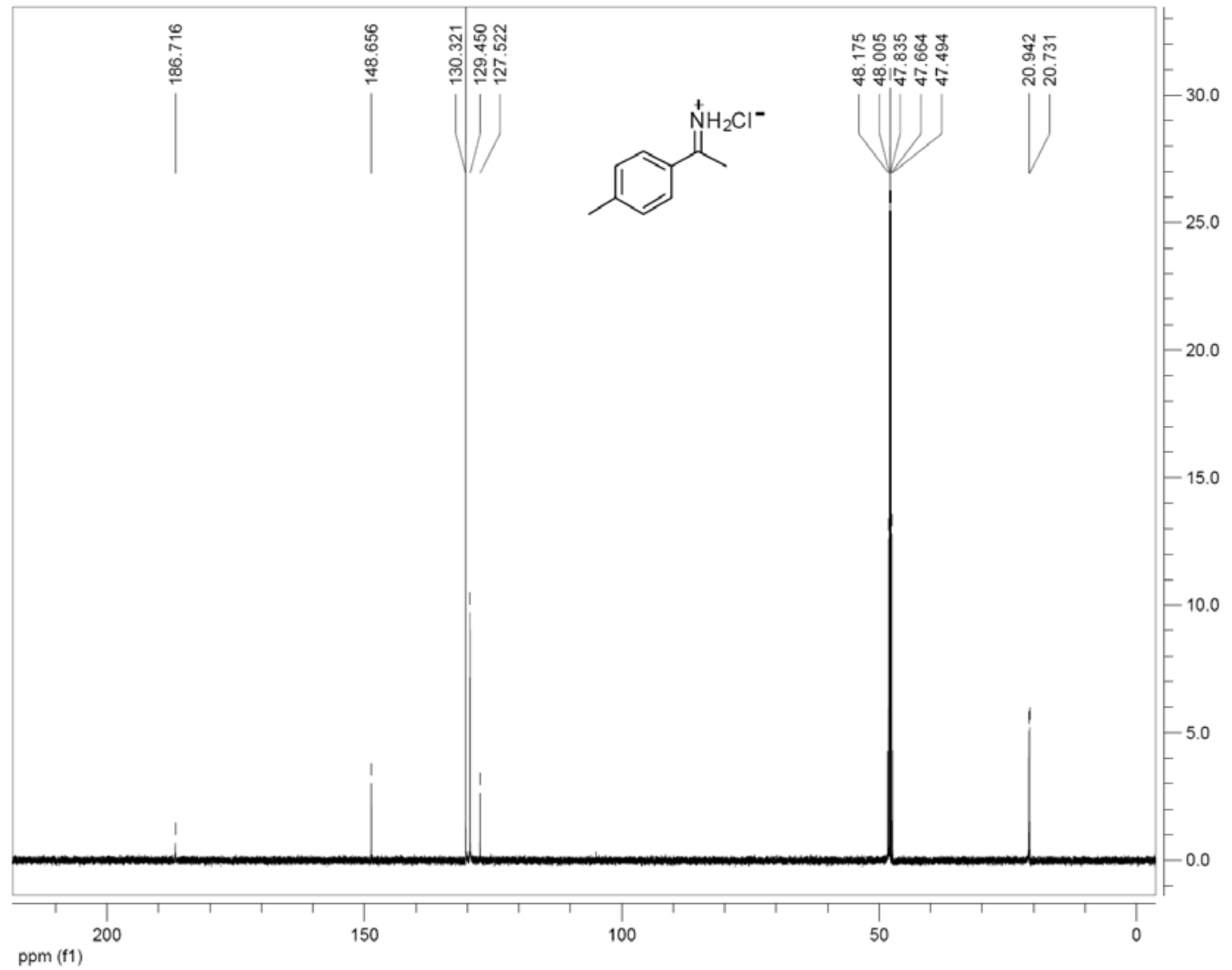

1-(4-Methoxyphenyl)ethaniminium chloride (3f)

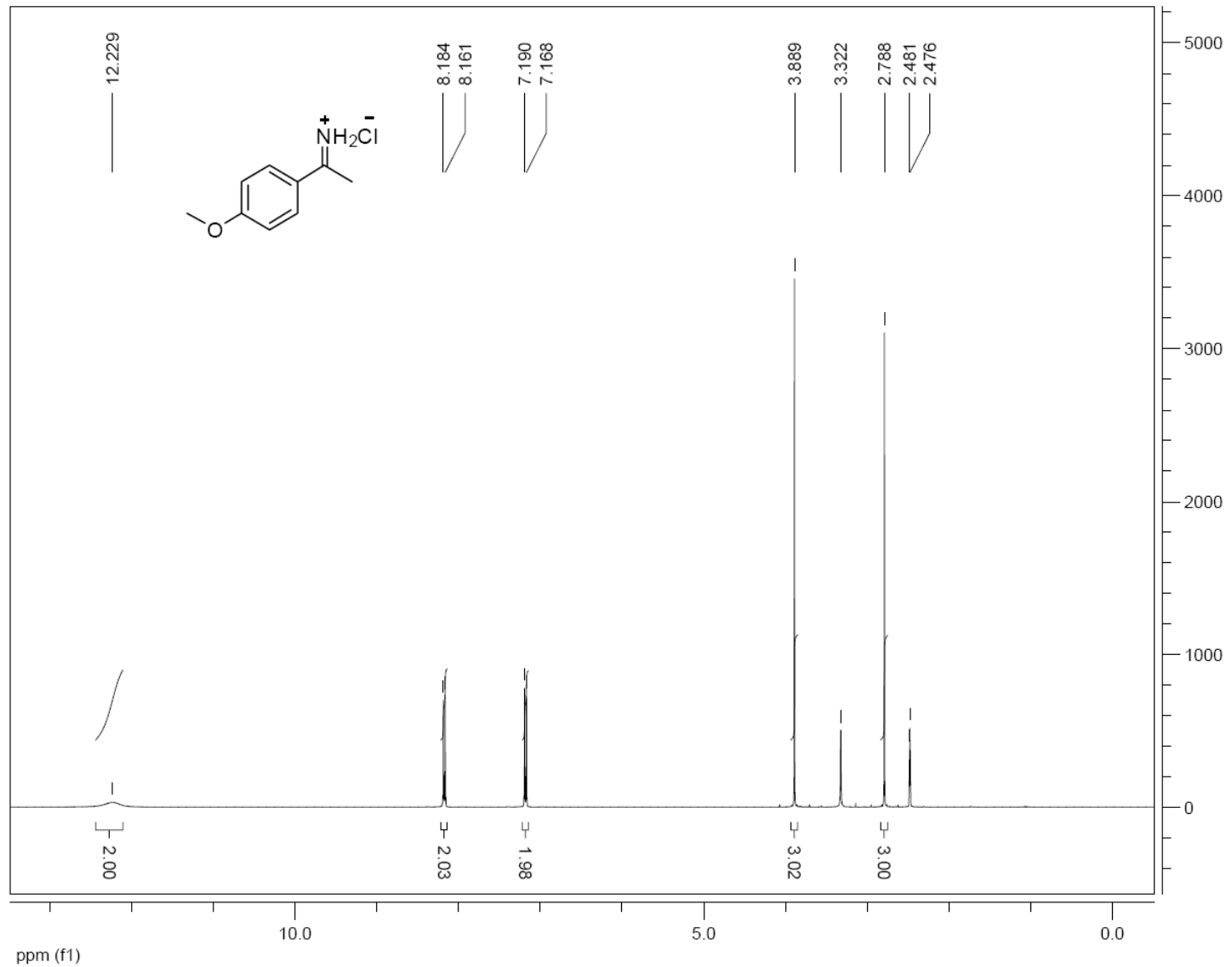




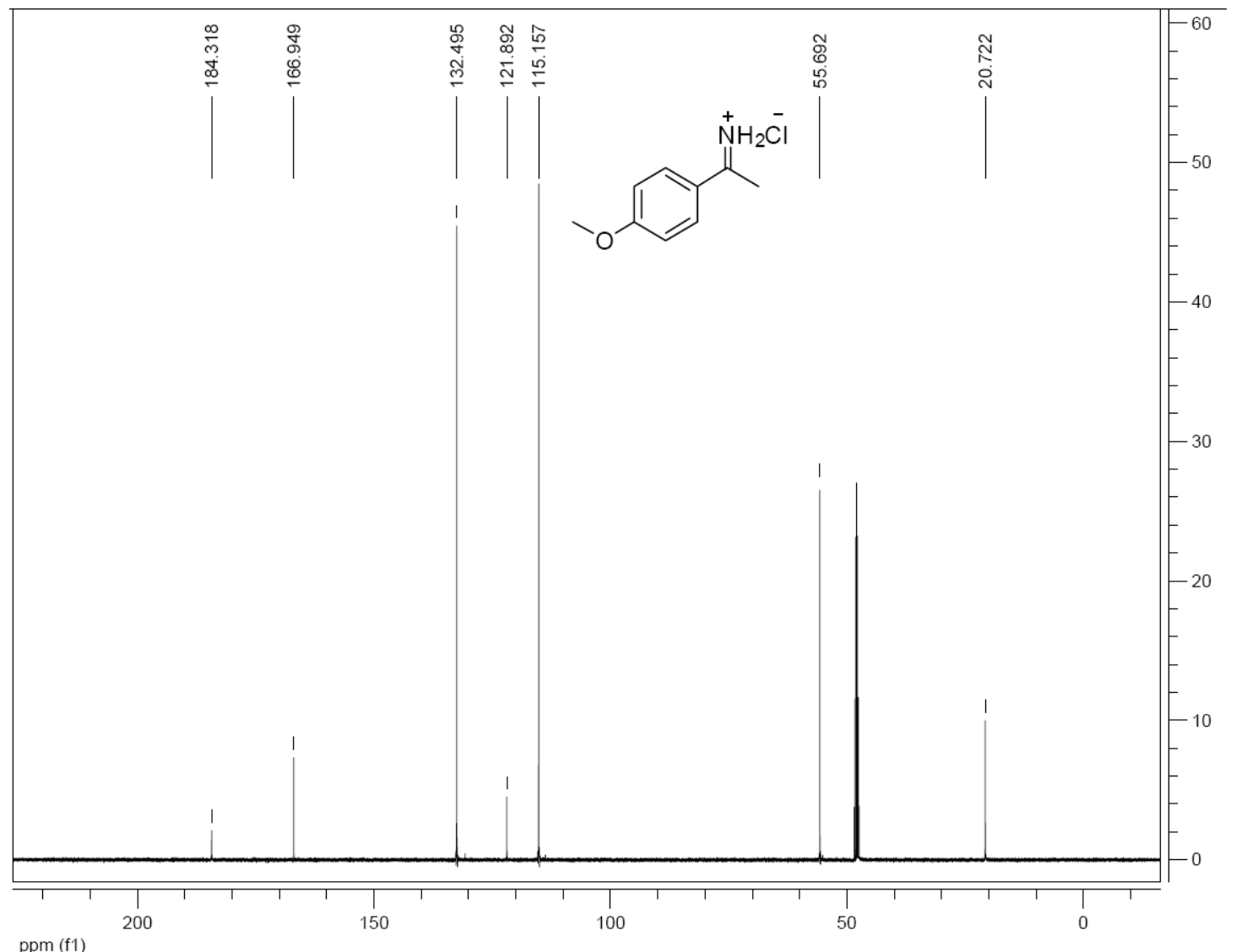

1-(4-Fluorophenyl)ethaniminium chloride (3g)

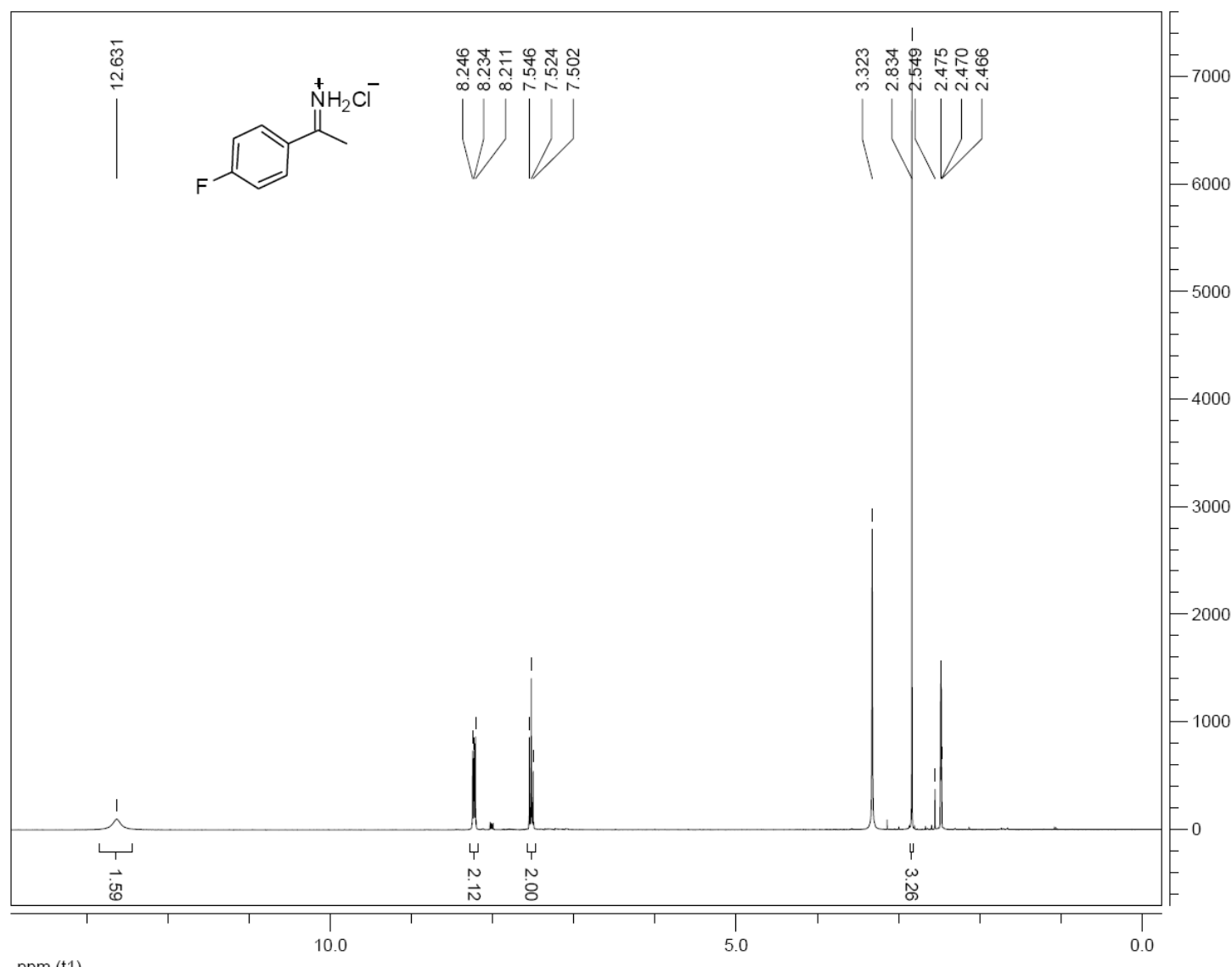

ppm (t1) 


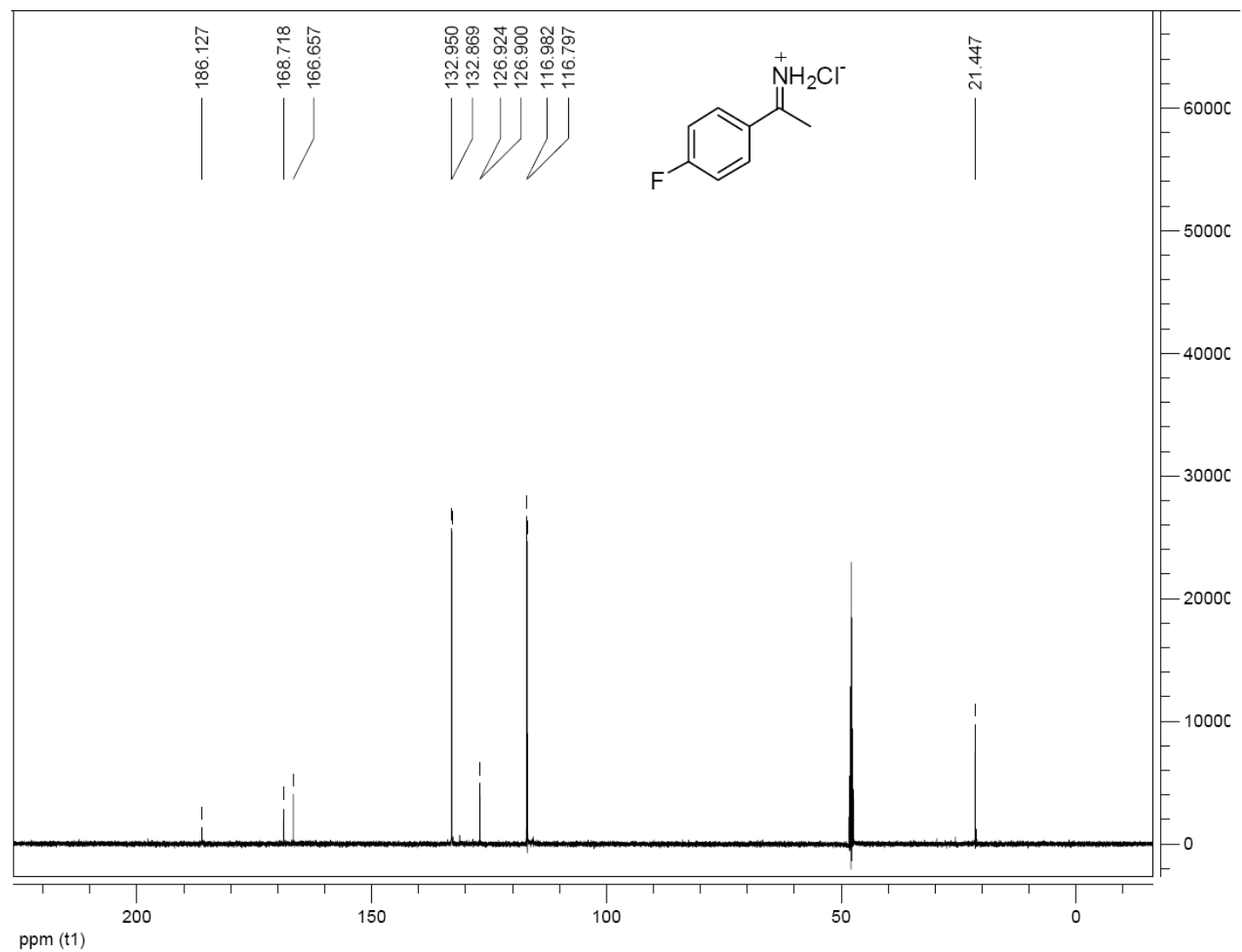

\section{1-(4-Chlorophenyl)ethaniminium chloride (3h)}

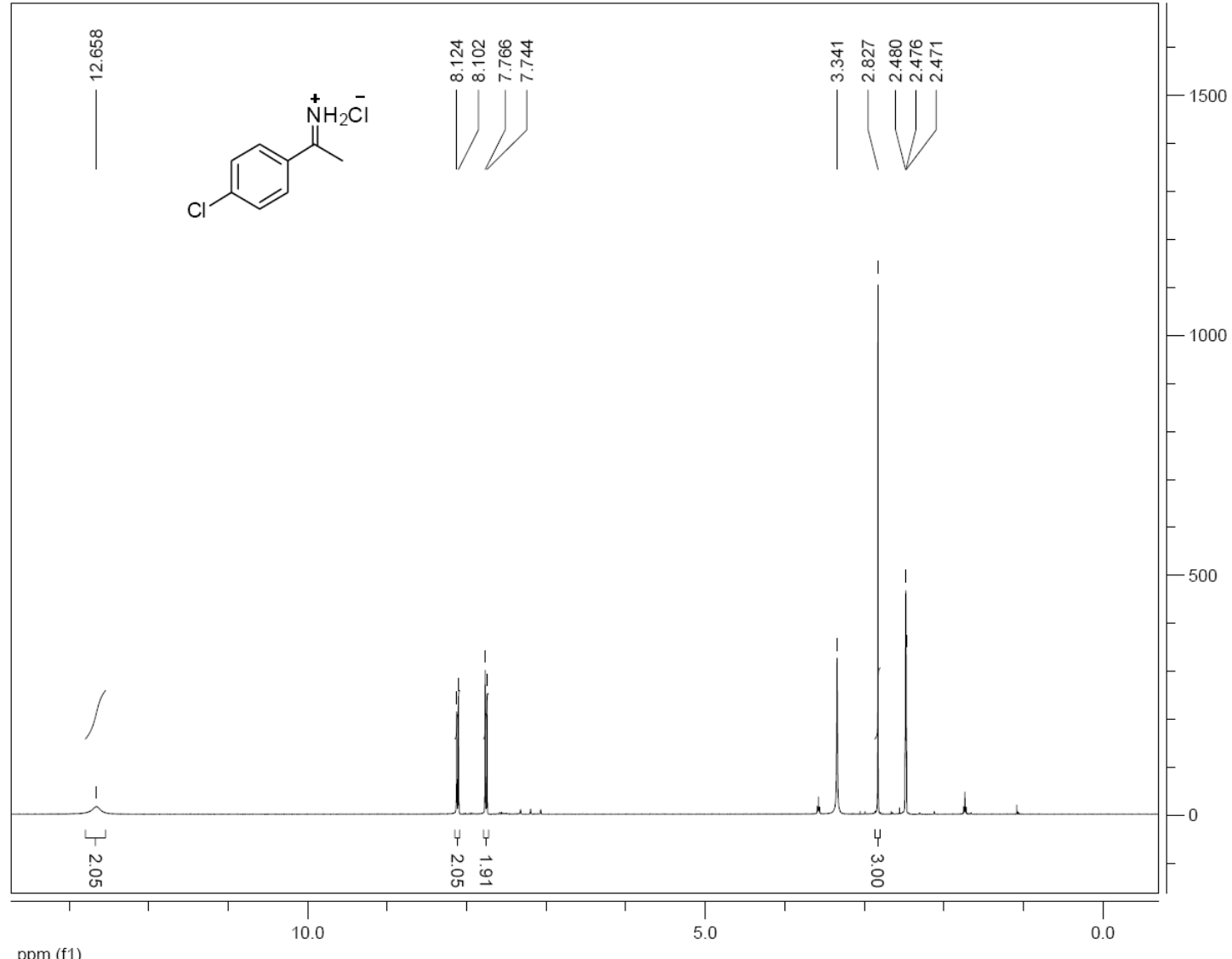




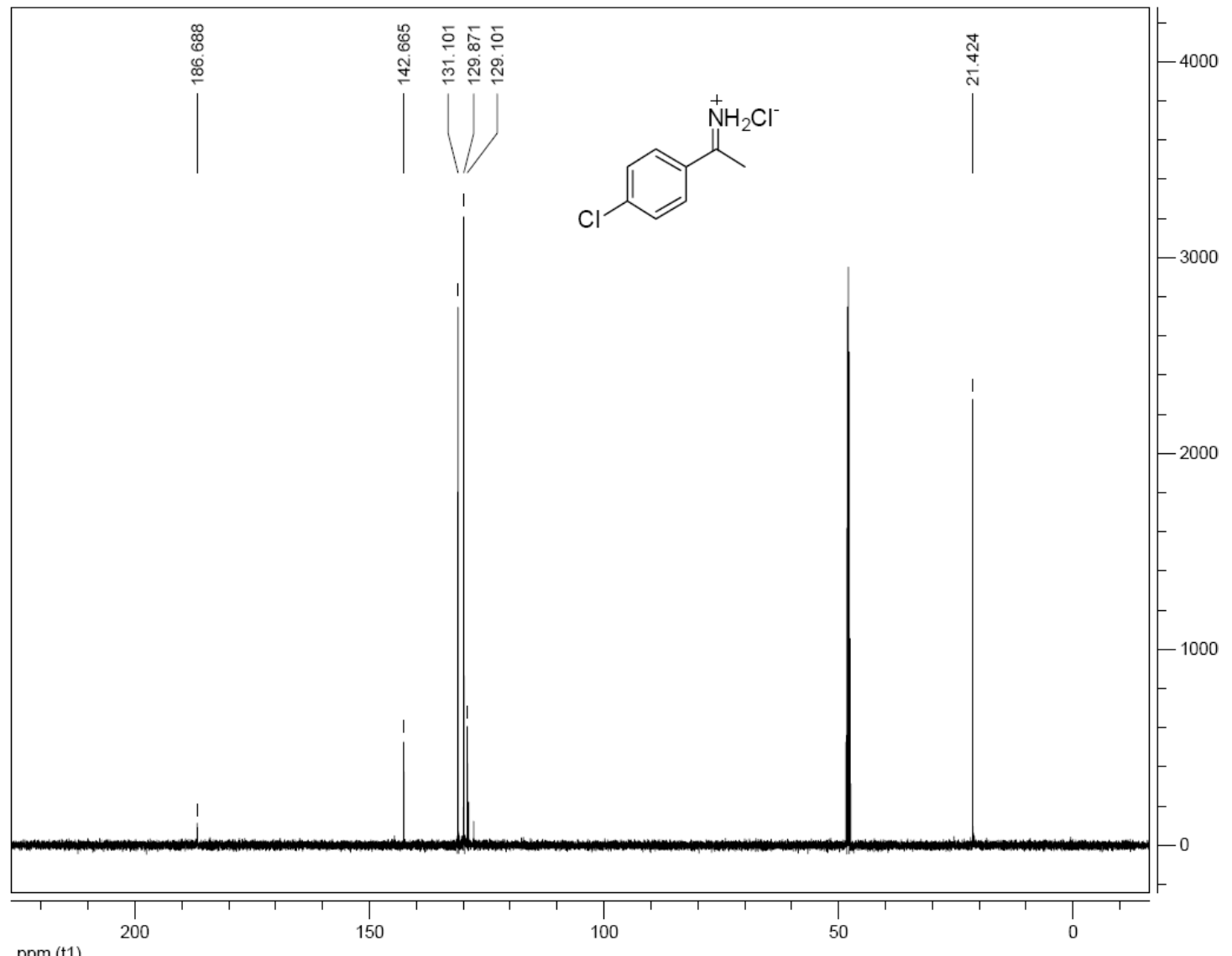

\section{1-(4-Bromophenyl)ethaniminium chloride (3i)}

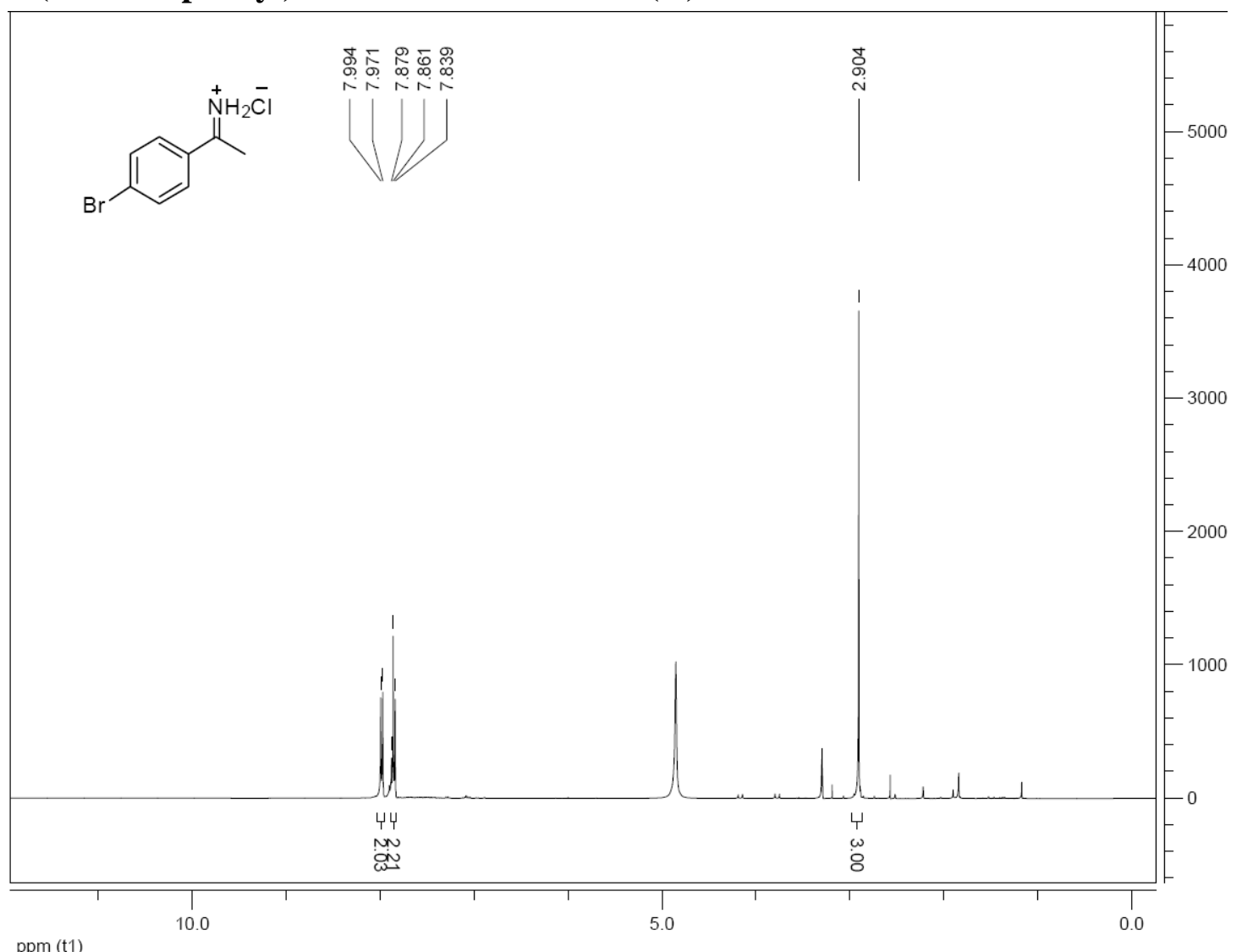




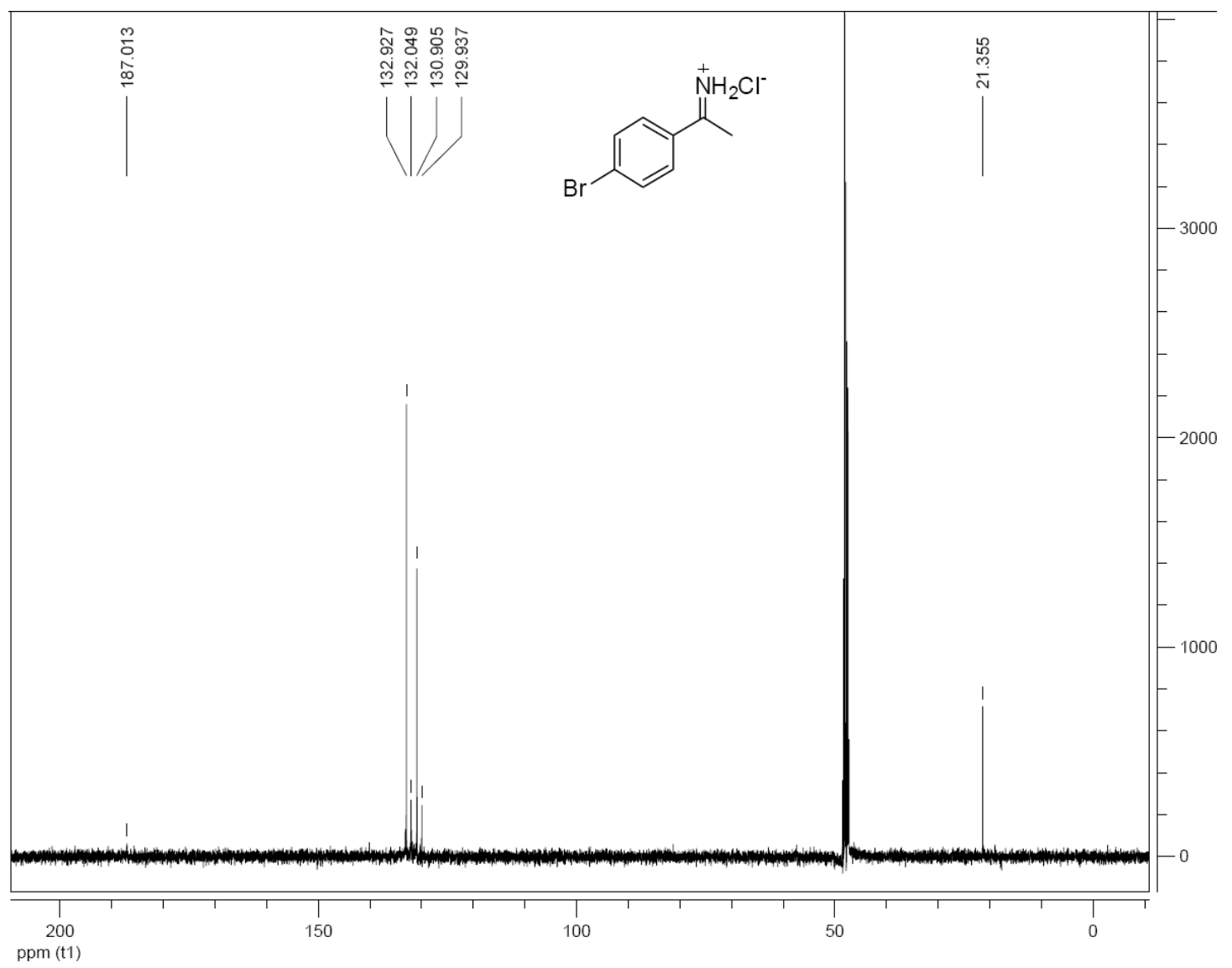

1-m-Tolylethaniminium chloride (3k)

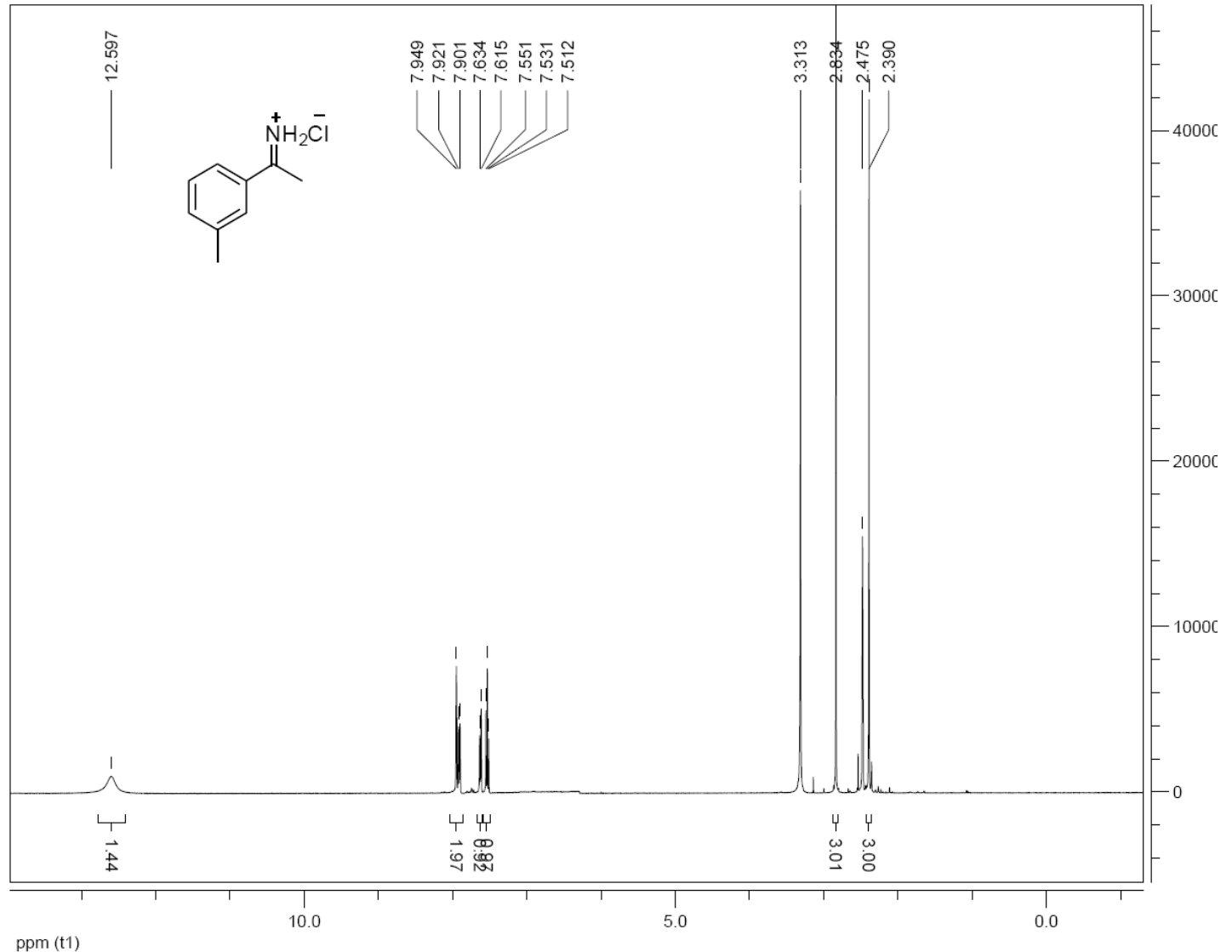




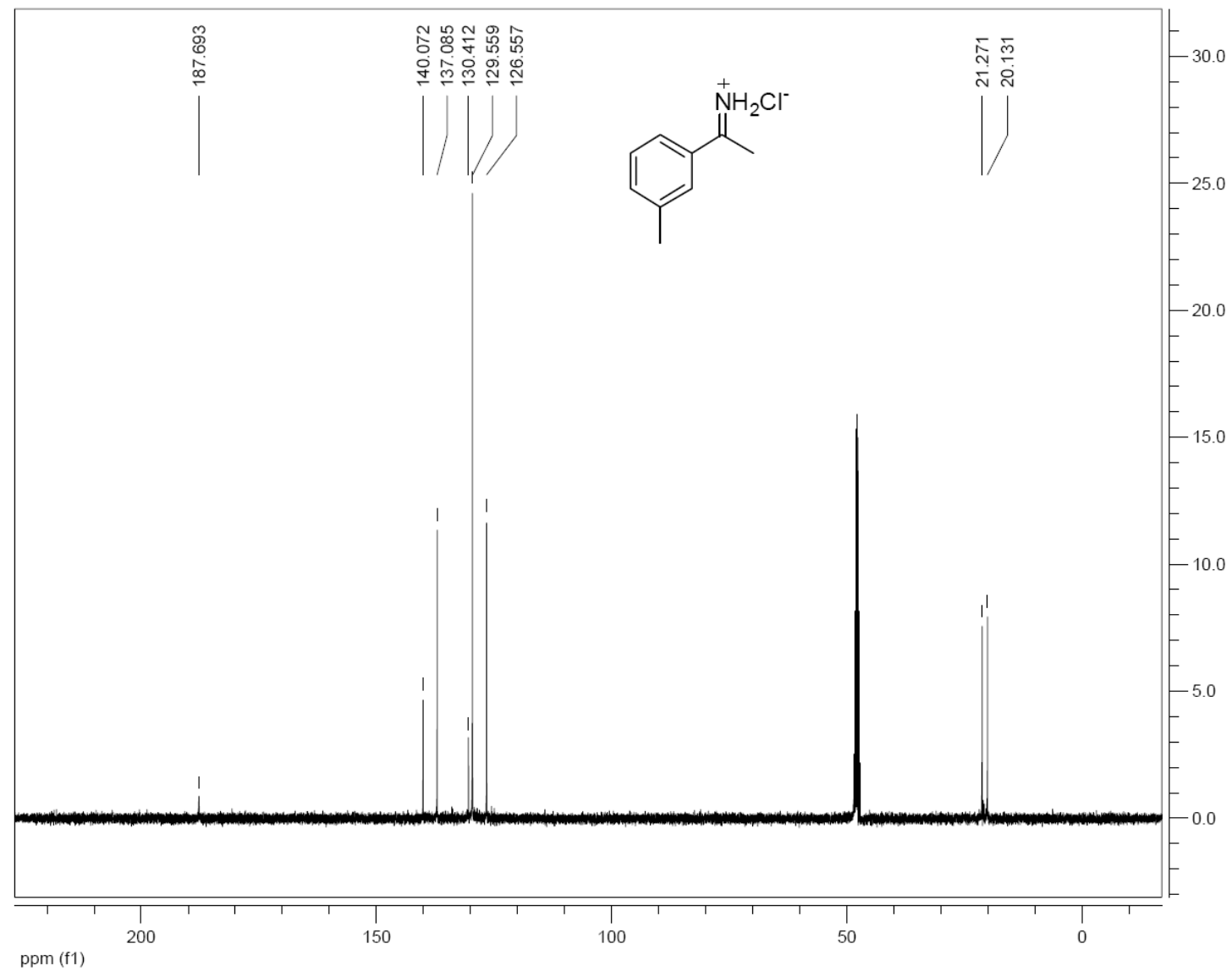

1-(3-Methoxyphenyl)ethaniminium chloride (3l)

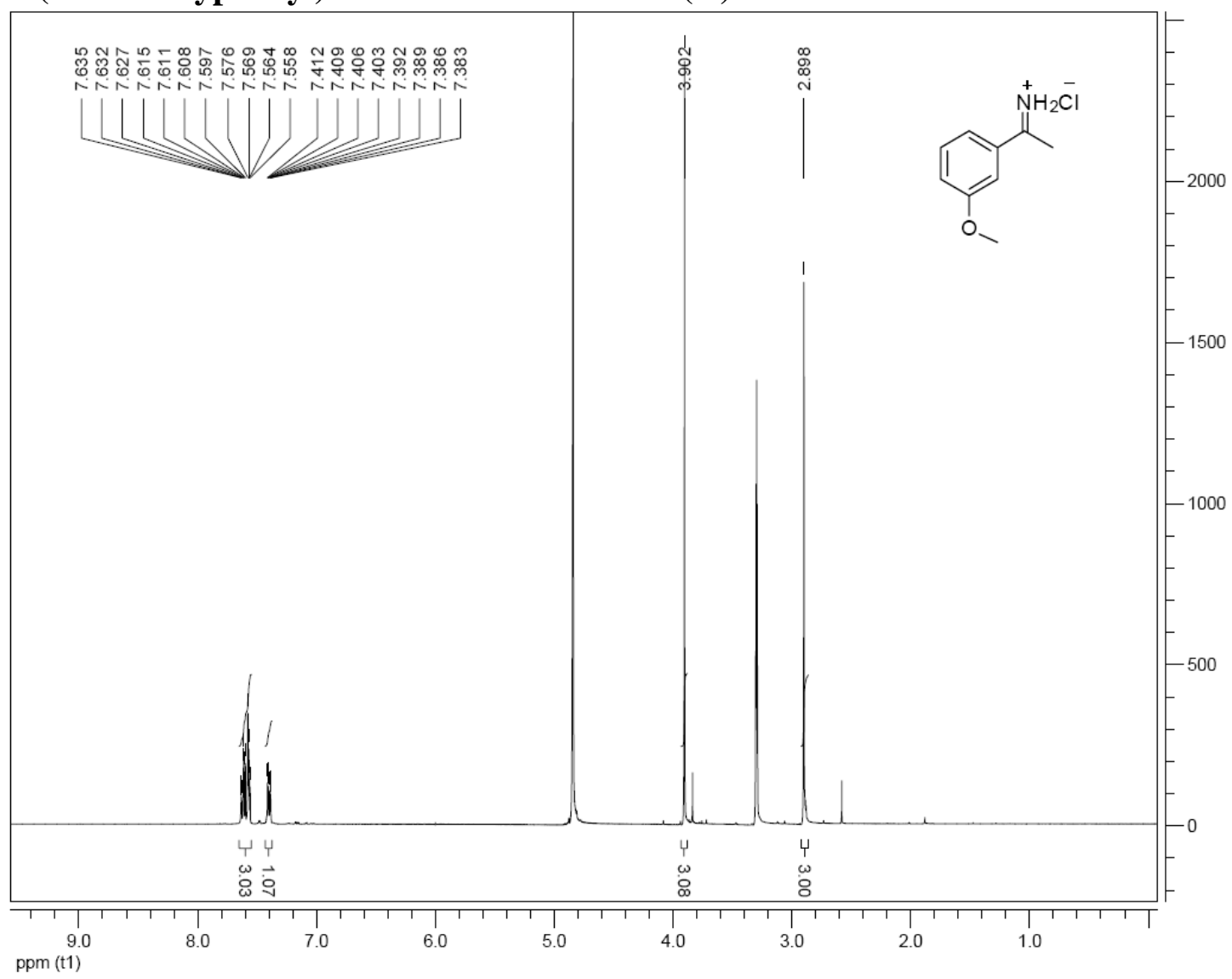




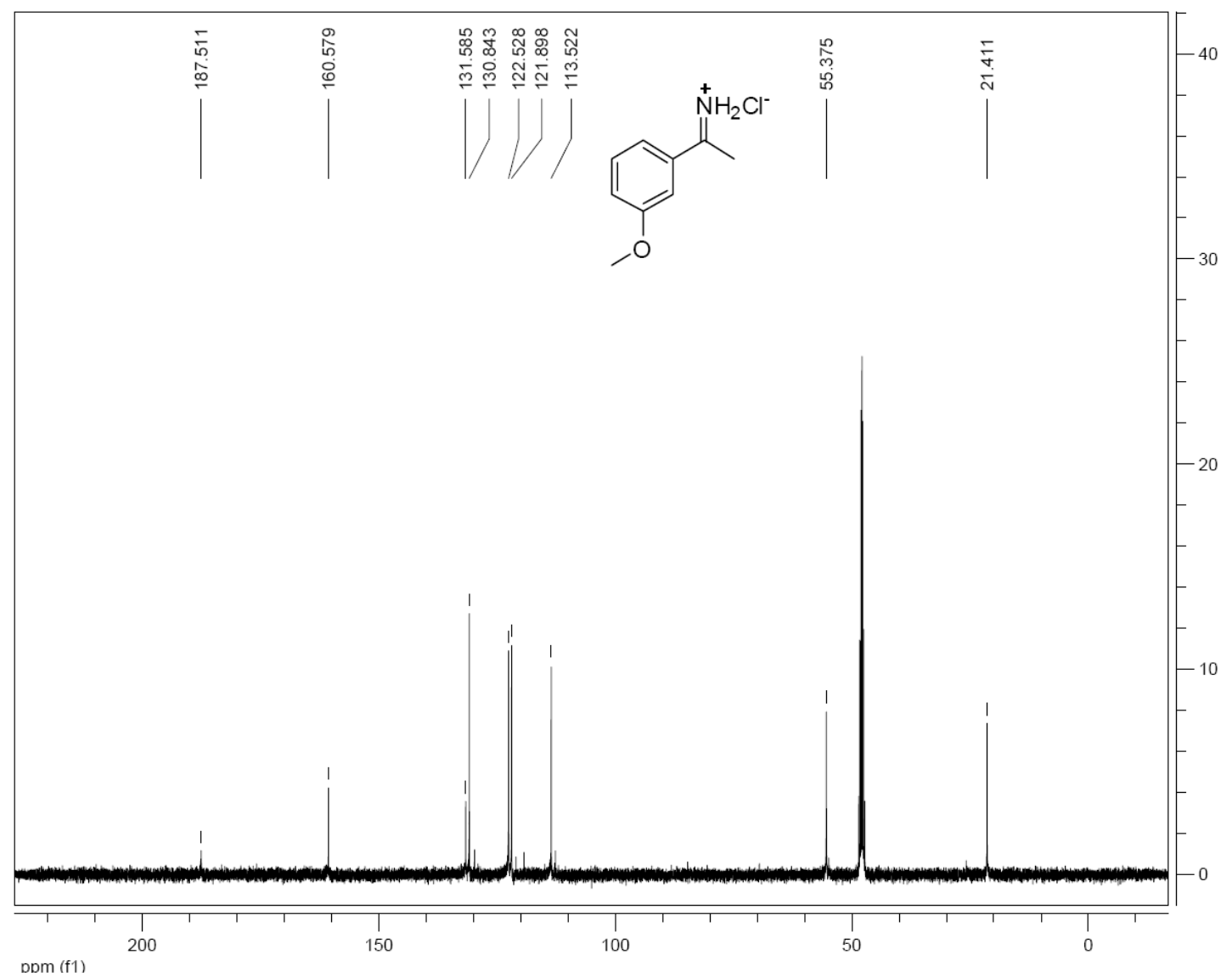

1-o-Tolylethaniminium chloride (3o)

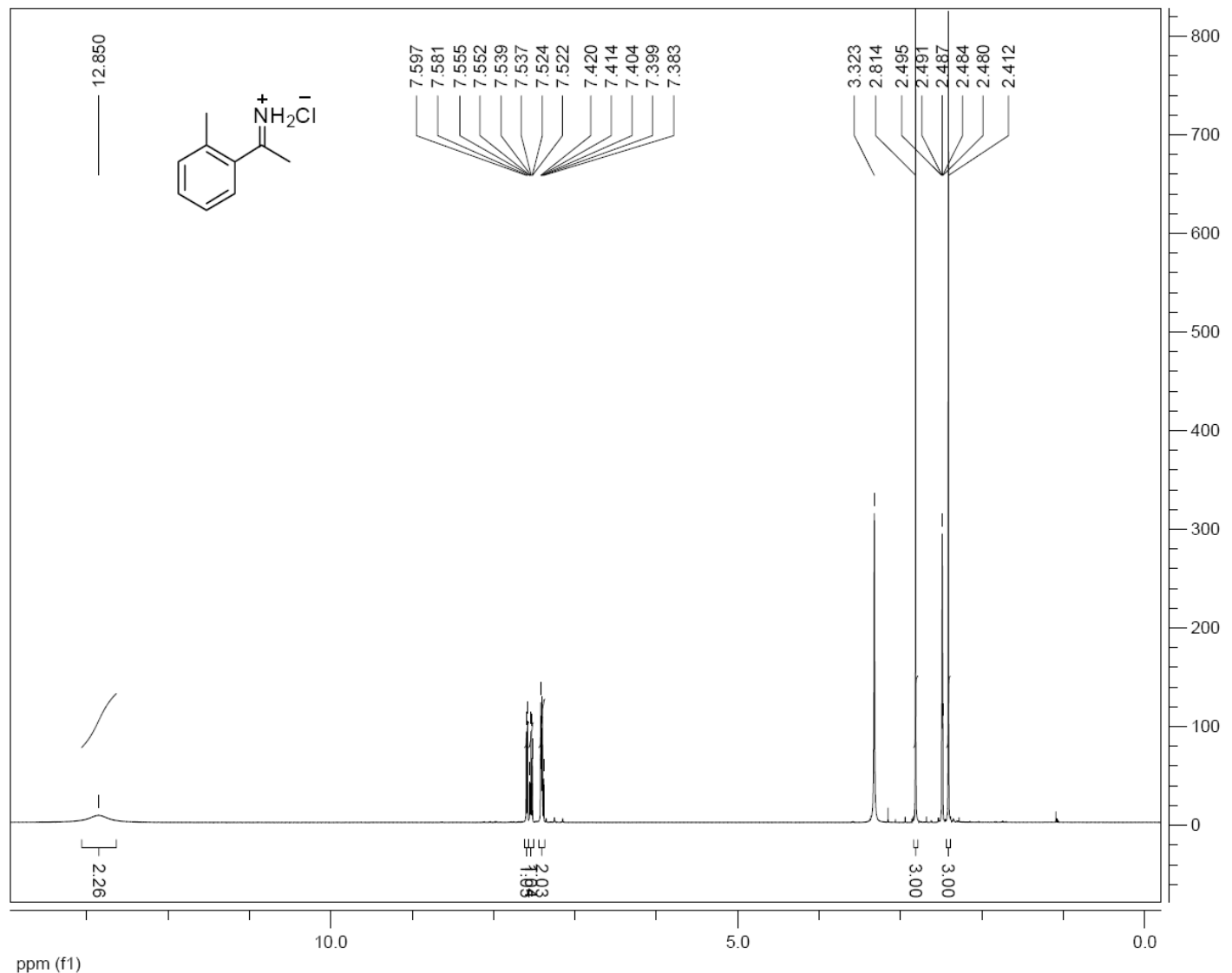




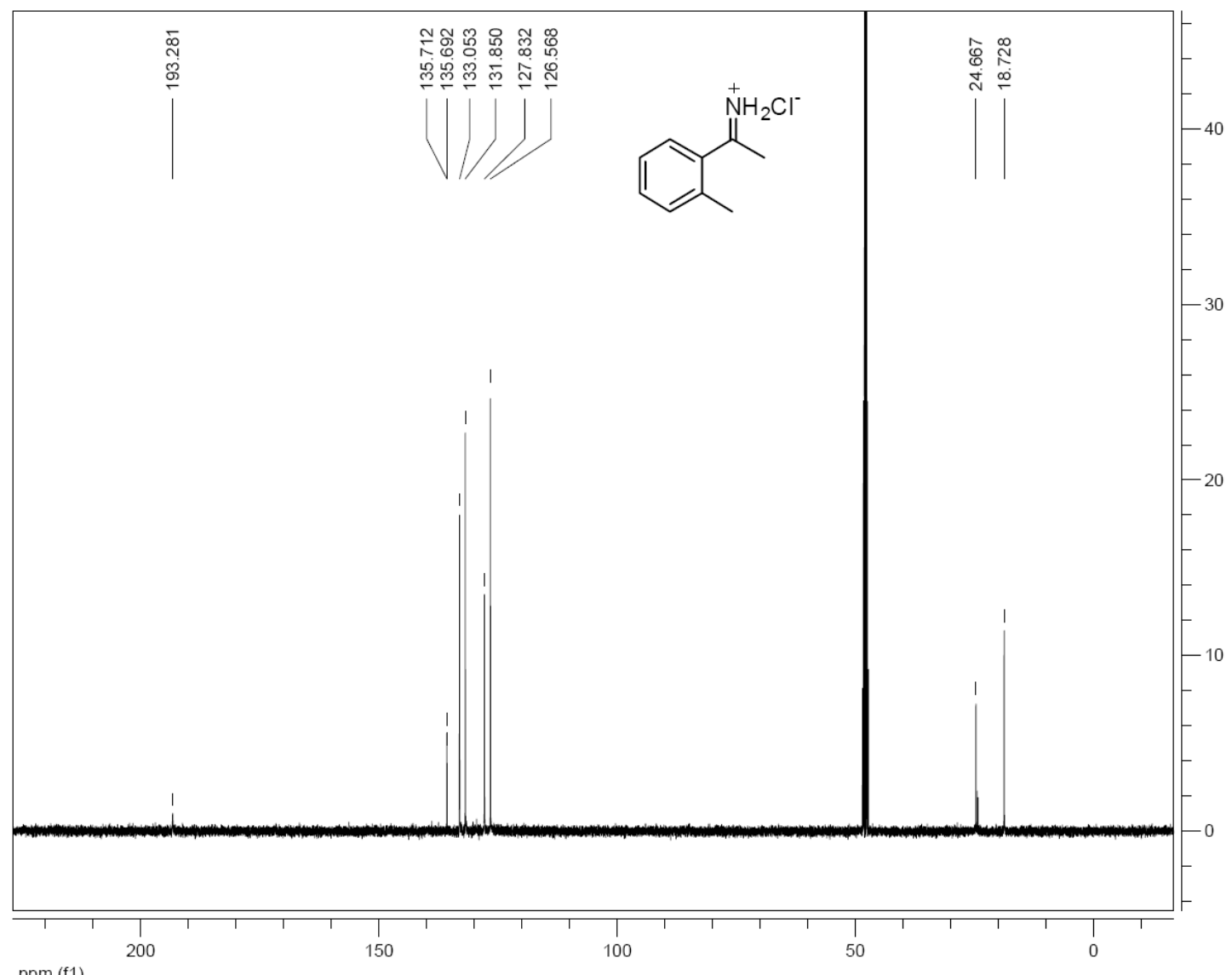

1-(2-Methoxyphenyl)ethaniminium chloride (3p)

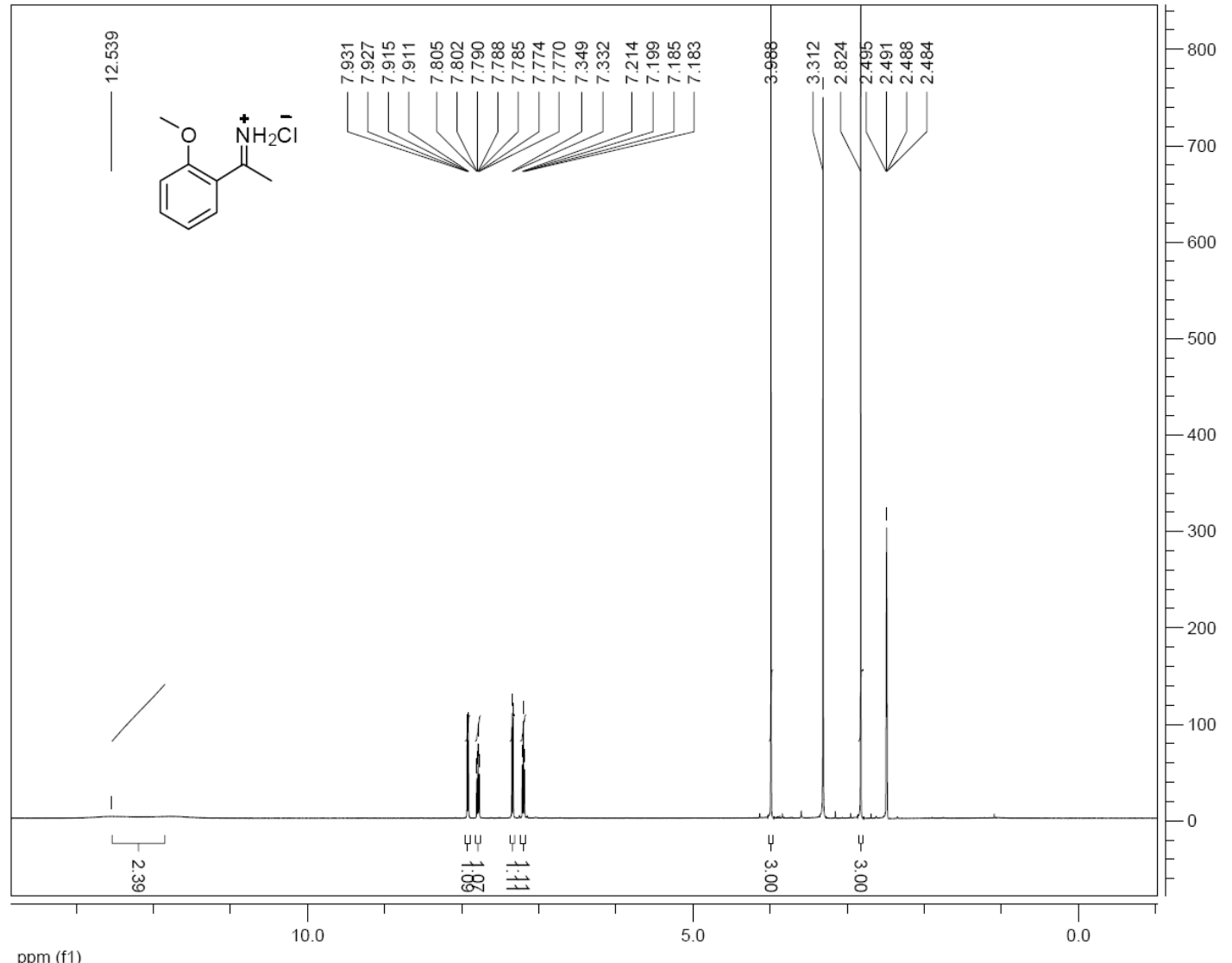



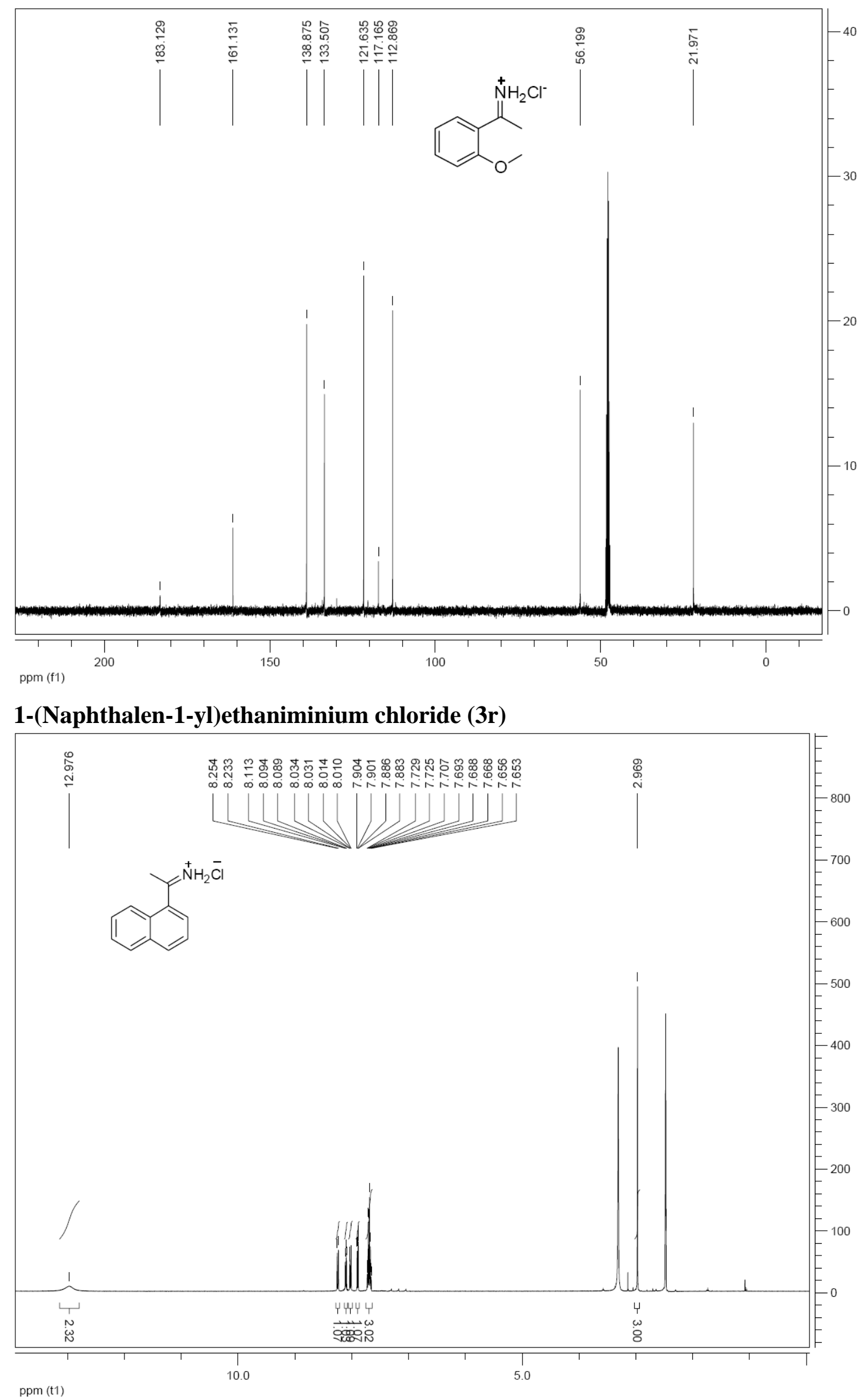

S21 


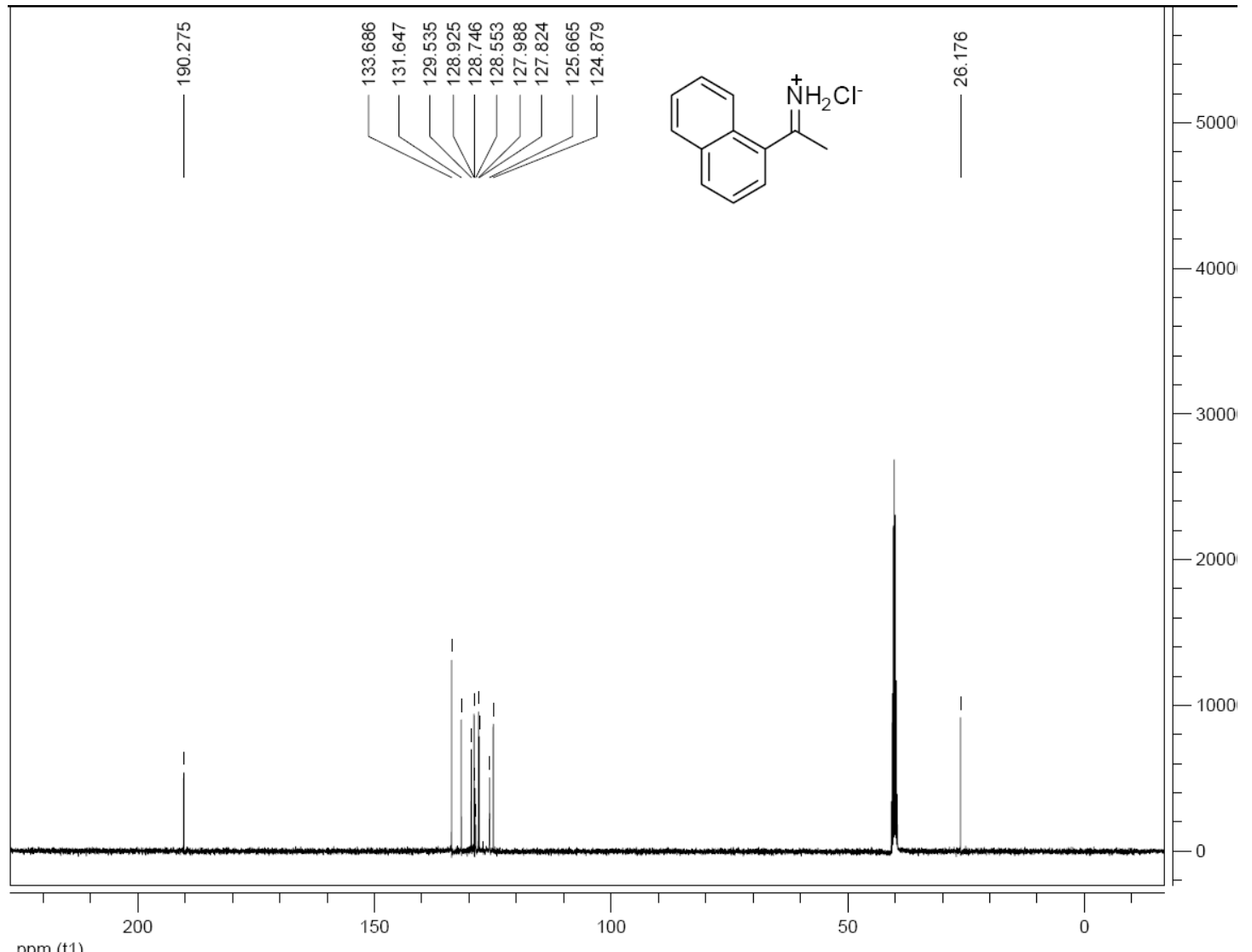

ppm (t1)

1-(Naphthalen-2-yl)ethaniminium chloride (3s)

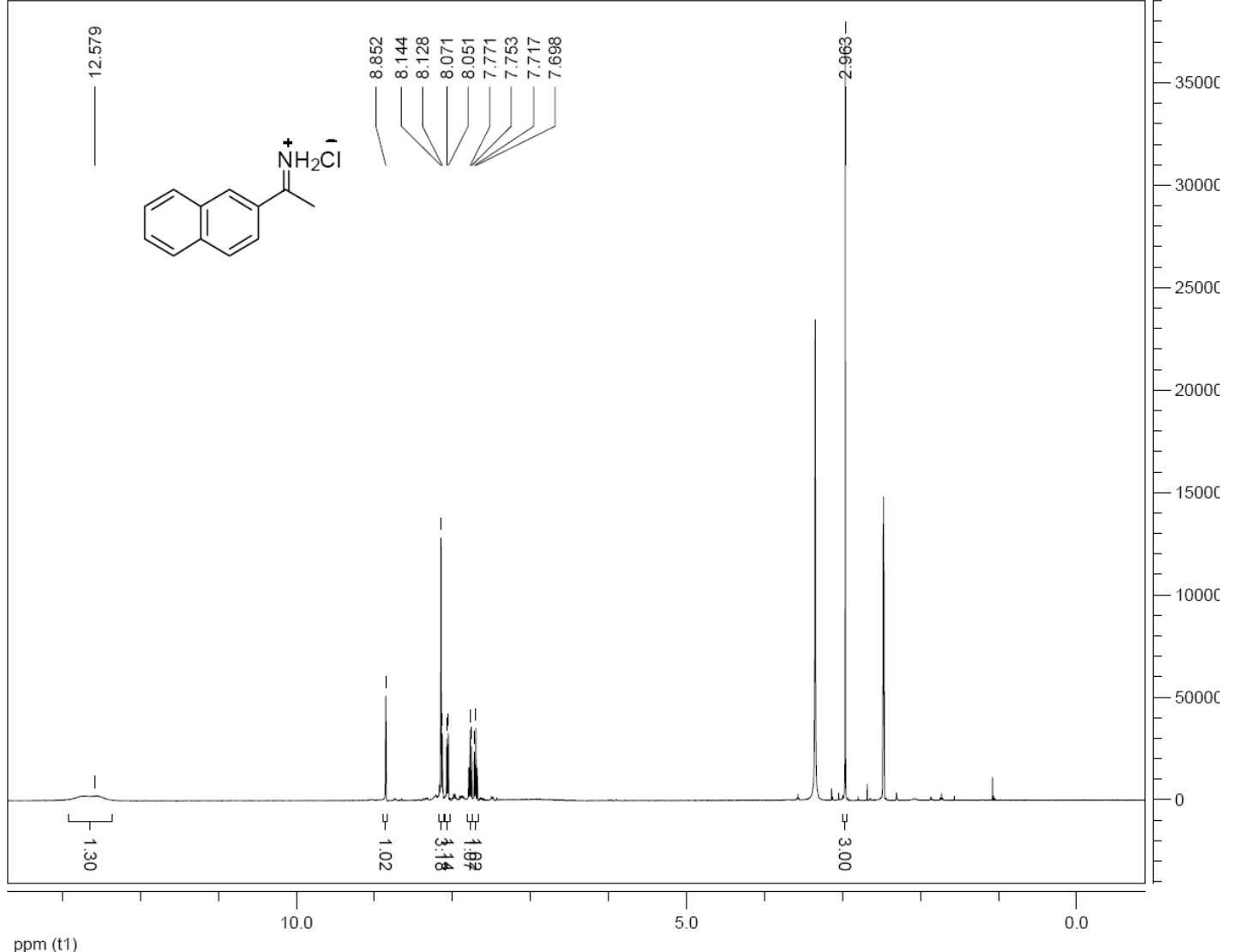




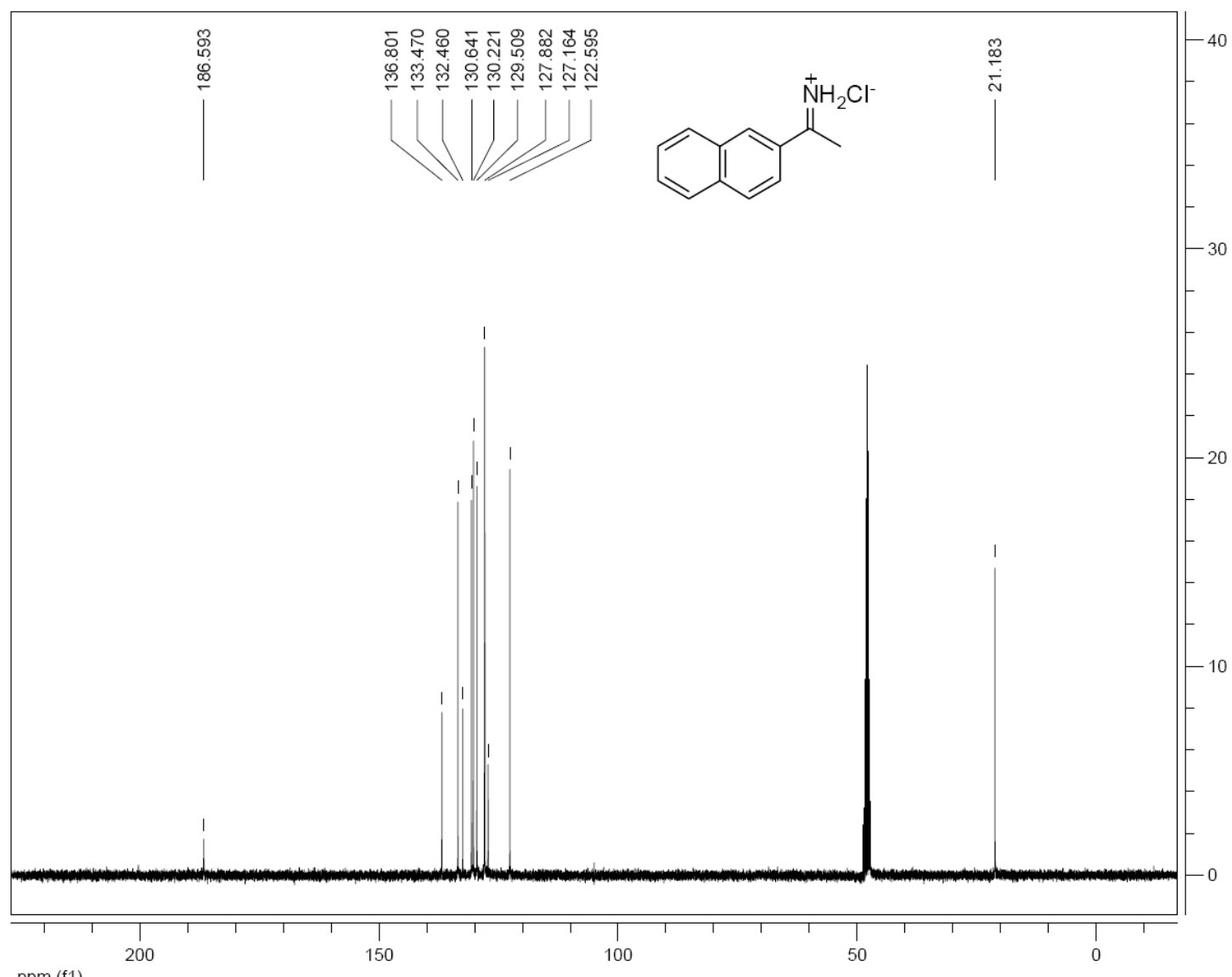

ppm (f1)

3,3-Dimethylbutan-2-iminium chloride (3t)

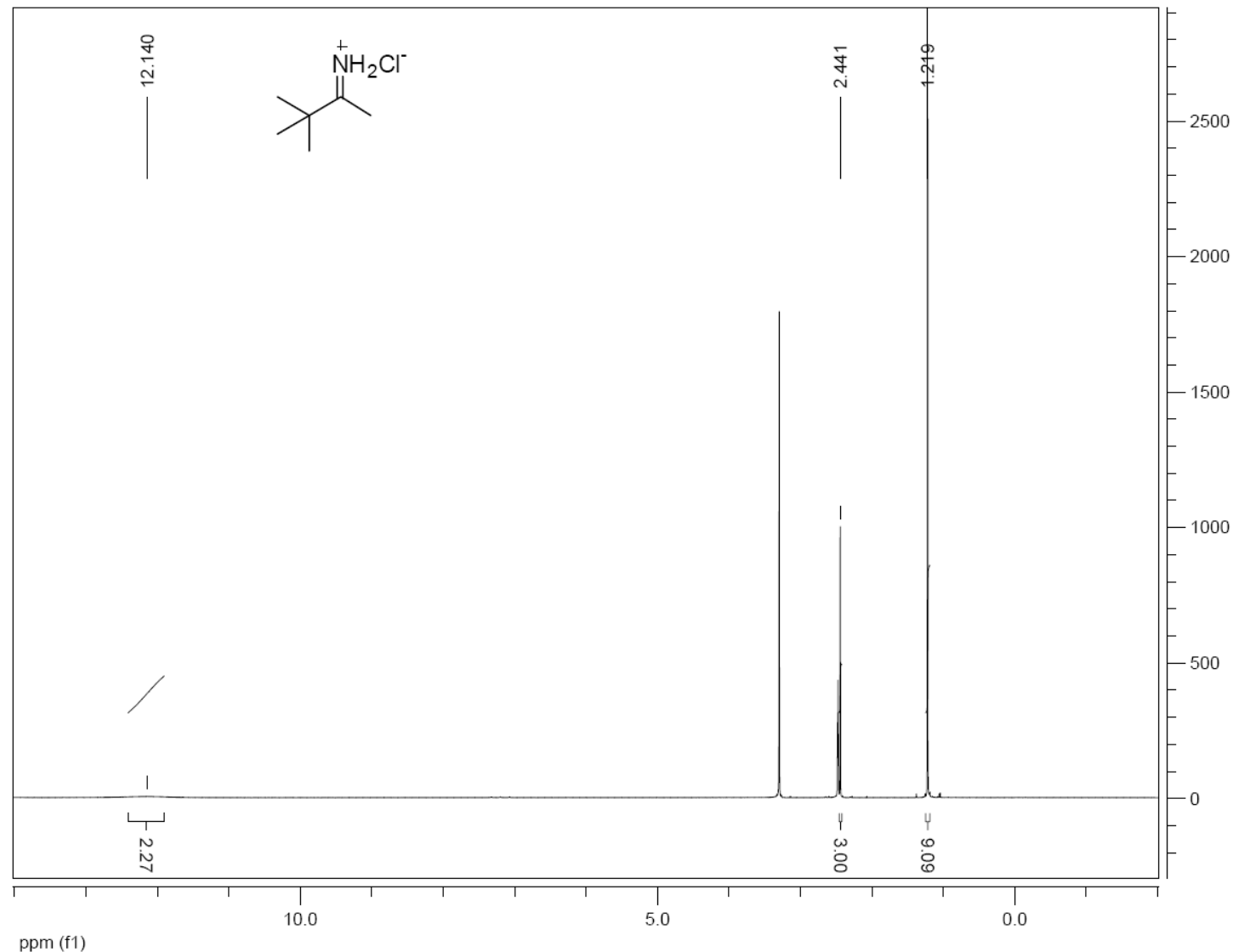




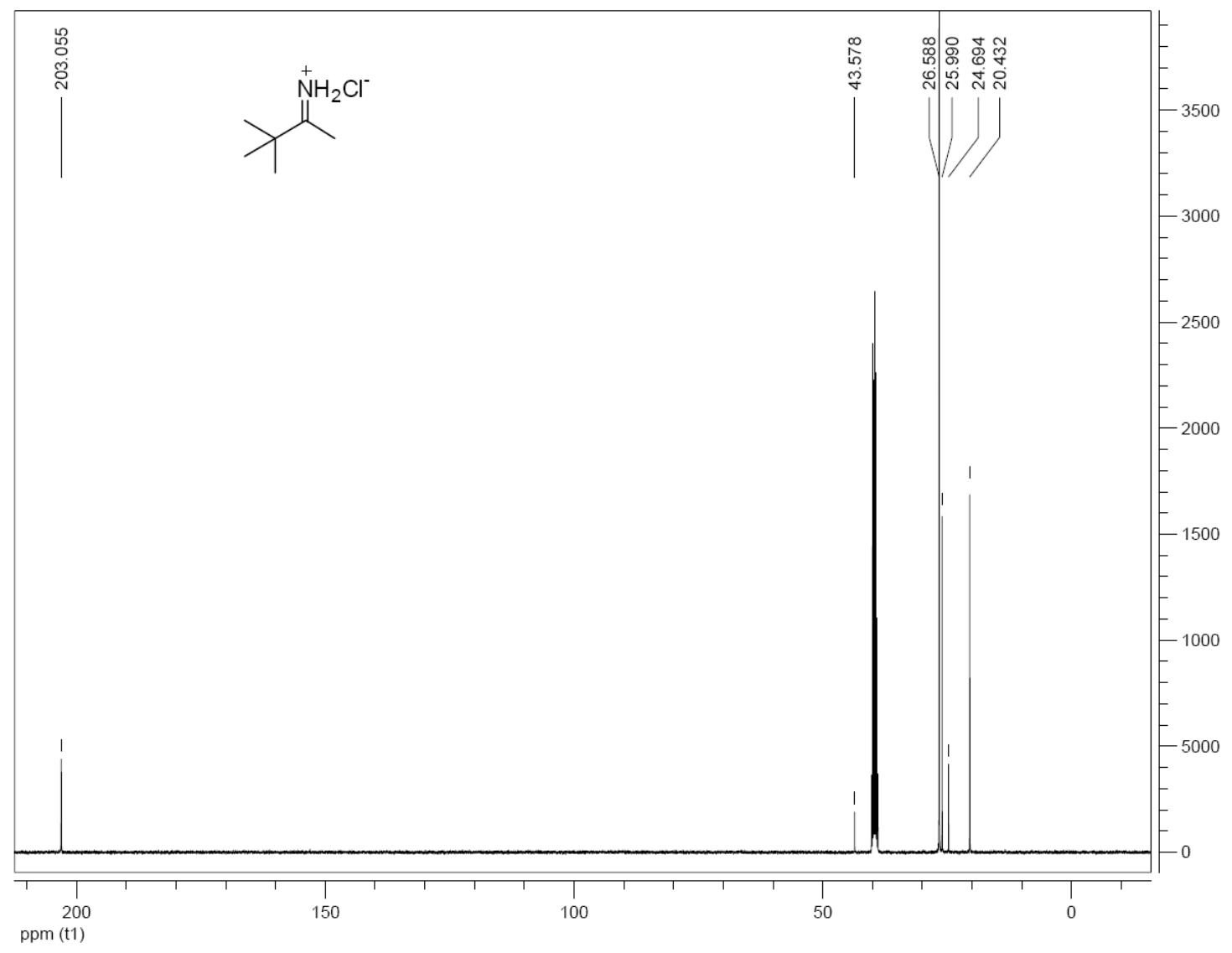

\section{1-Cyclohexylethaniminium chloride (3u)}

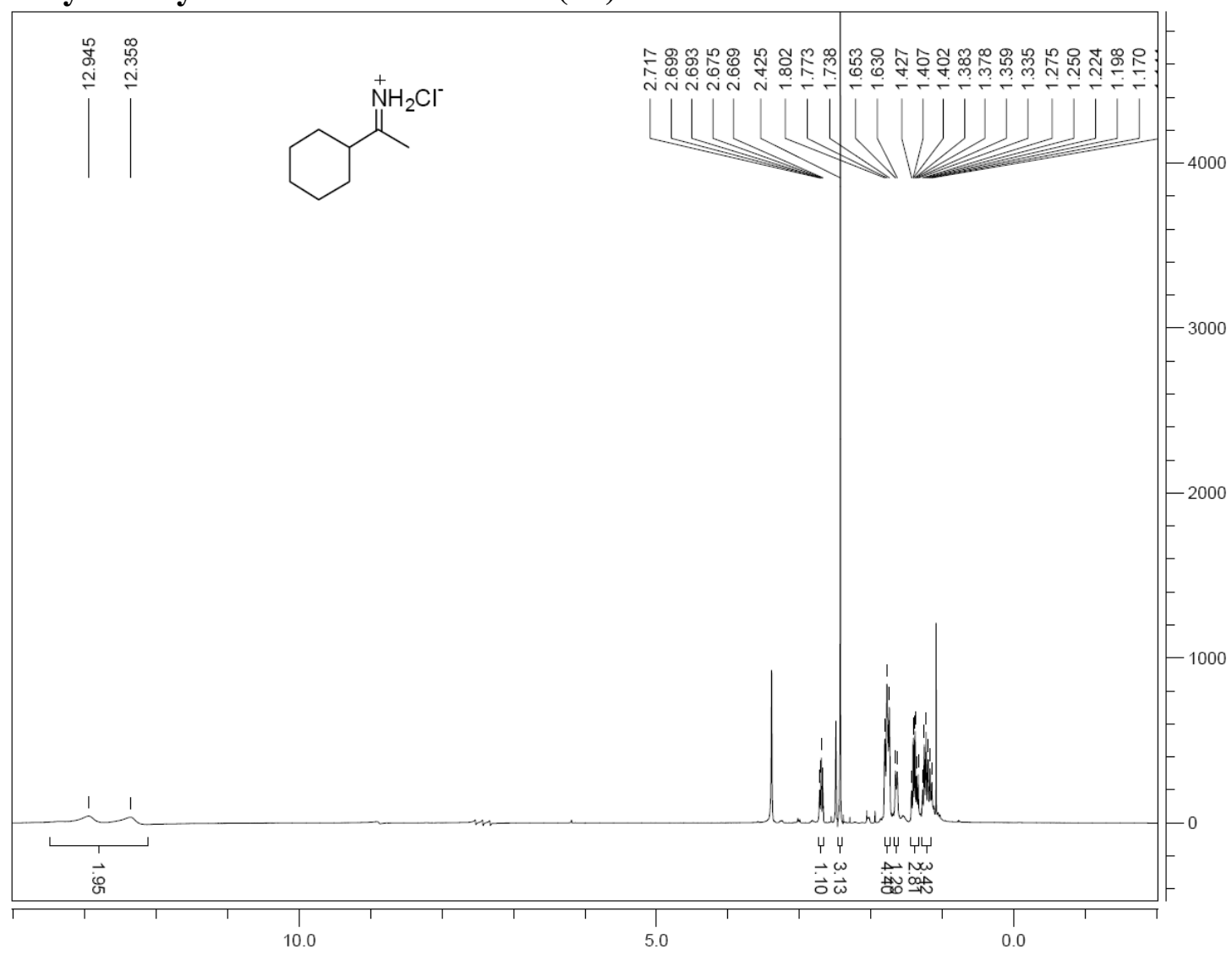

ppm (t1) 


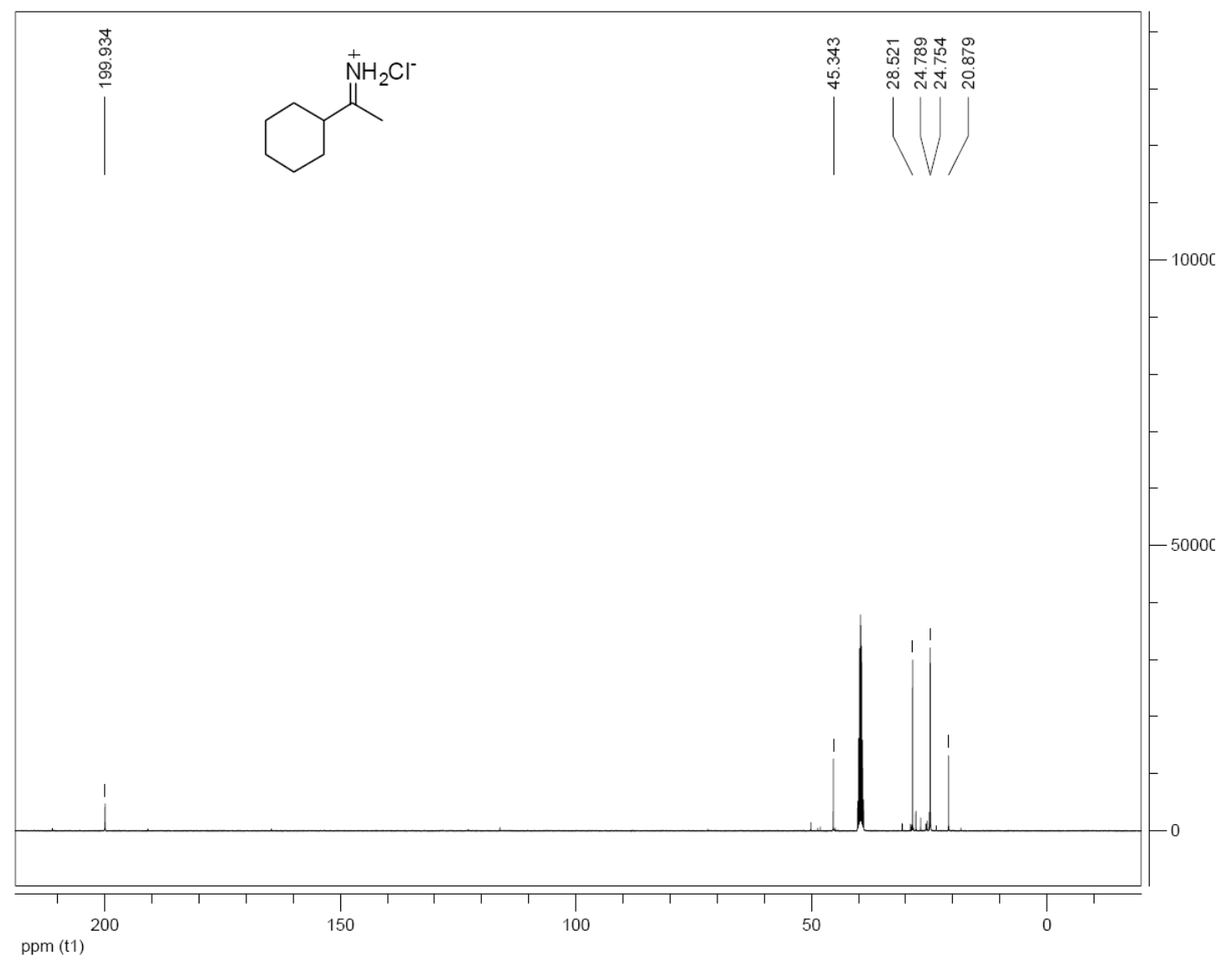

(F) GC Chromatograms for Hydrogenation Products

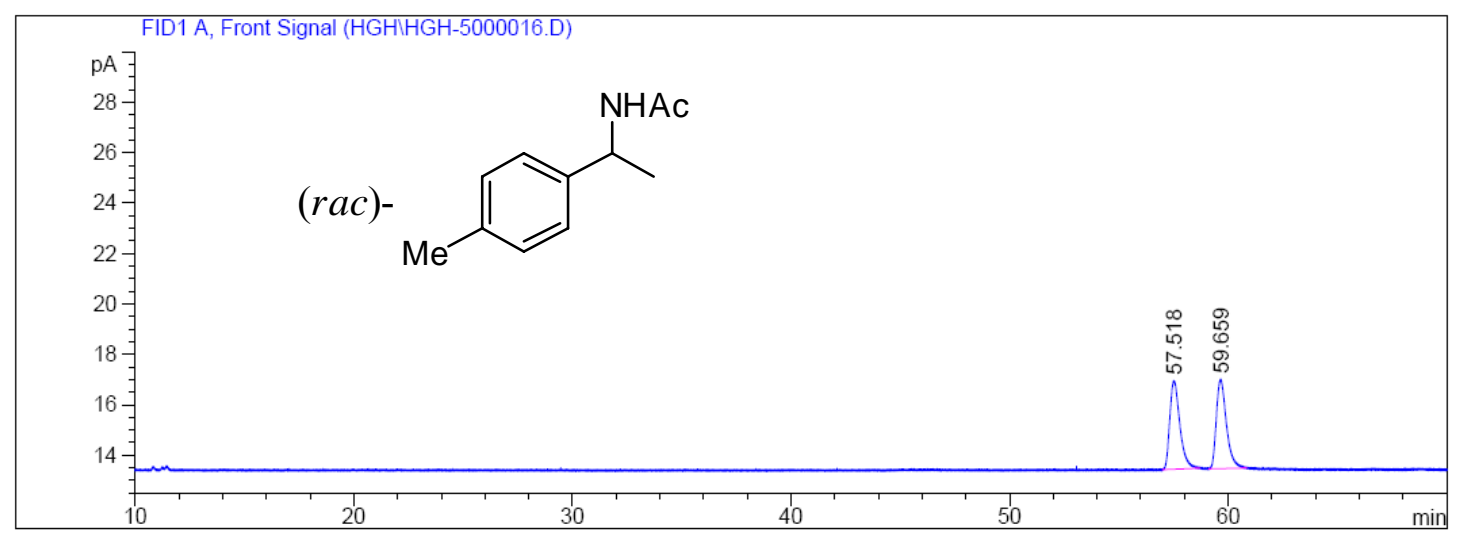



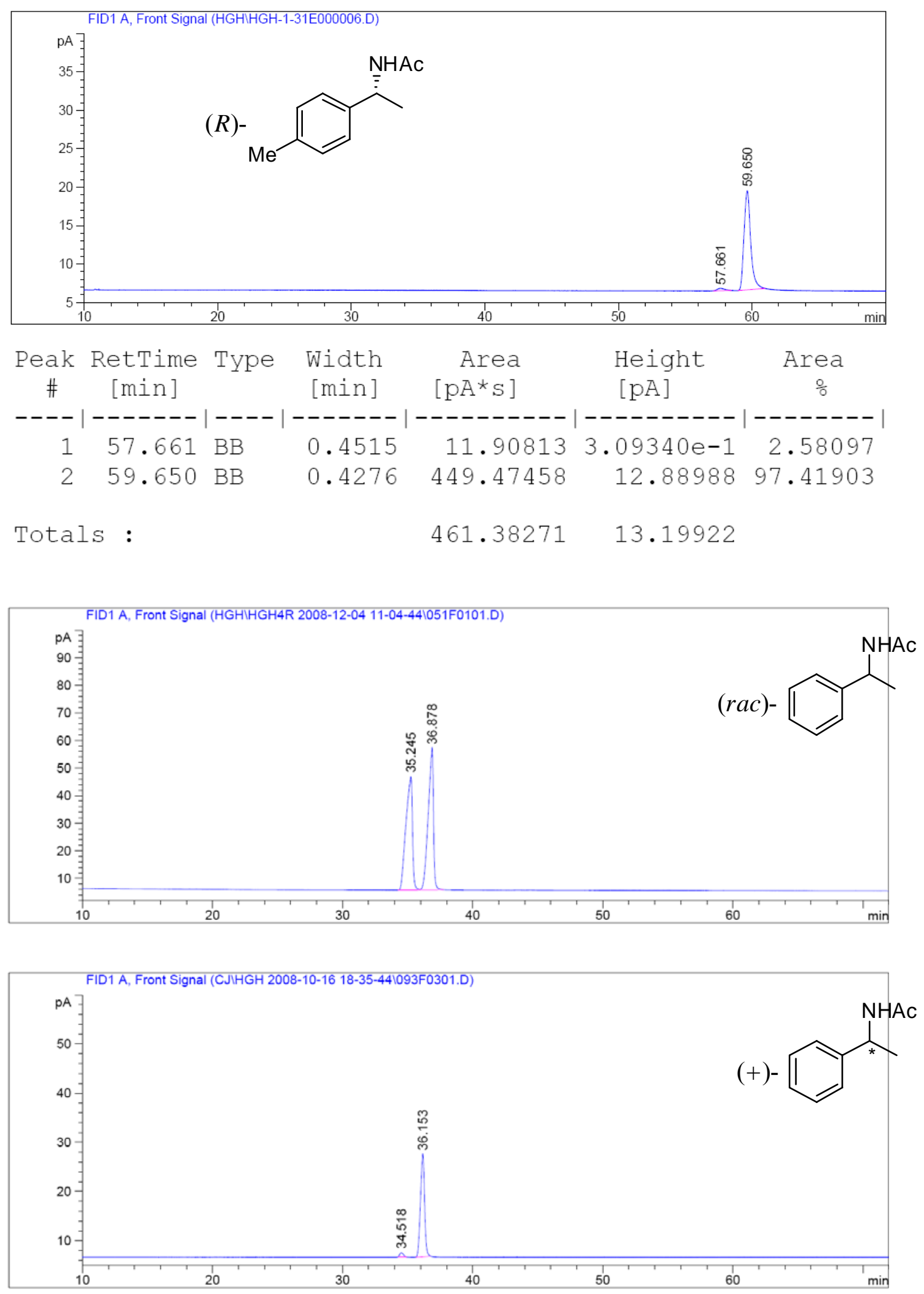


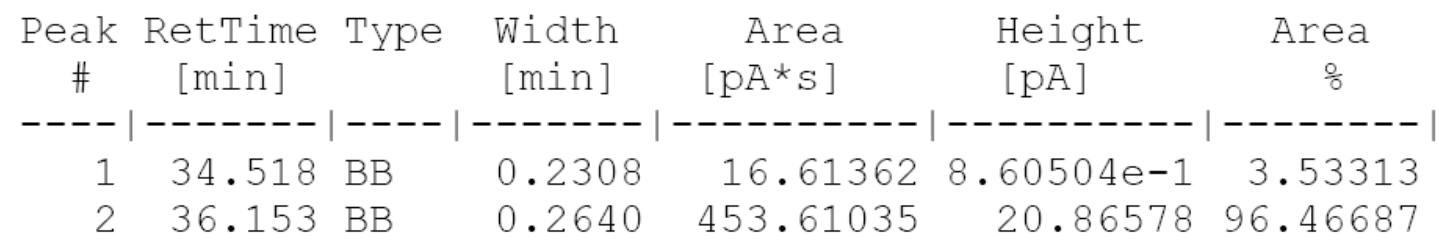

Totals :

$470.22398 \quad 21.72628$
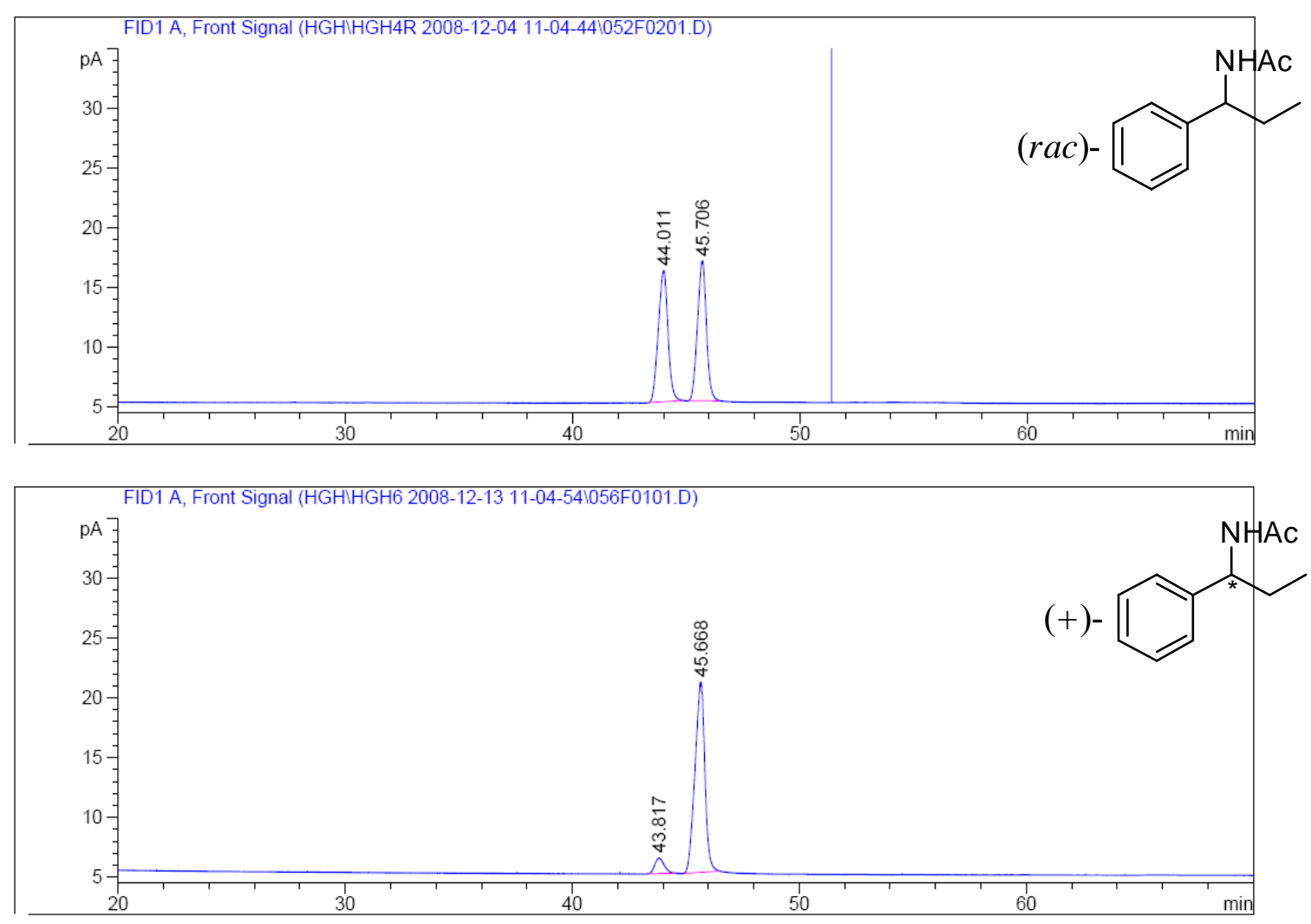

\begin{tabular}{|c|c|c|c|c|c|c|}
\hline $\begin{array}{c}\text { Peak } \\
\#\end{array}$ & $\begin{array}{c}\text { RetTime } \\
\text { [min] }\end{array}$ & Type & $\begin{array}{l}\text { Width } \\
\text { [min] }\end{array}$ & $\begin{array}{c}\text { Area } \\
{\left[\mathrm{pA}^{\star} \mathrm{s}\right]}\end{array}$ & $\begin{array}{l}\text { Height } \\
{[\mathrm{pA}]}\end{array}$ & $\begin{array}{c}\text { Area } \\
\frac{\circ}{0}\end{array}$ \\
\hline & 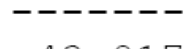 & & & 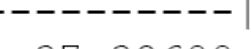 & & \\
\hline $\begin{array}{l}1 \\
2\end{array}$ & $\begin{array}{l}43.817 \\
45.668\end{array}$ & $\begin{array}{l}\mathrm{BB} \\
\mathrm{BB}\end{array}$ & 0 . & $\begin{array}{r}37.32688 \\
480.35535\end{array}$ & $\begin{array}{l}29871 \\
90382\end{array}$ & $\begin{array}{l}1038 \\
8962\end{array}$ \\
\hline
\end{tabular}

Totals :

$517.68222 \quad 17.20253$

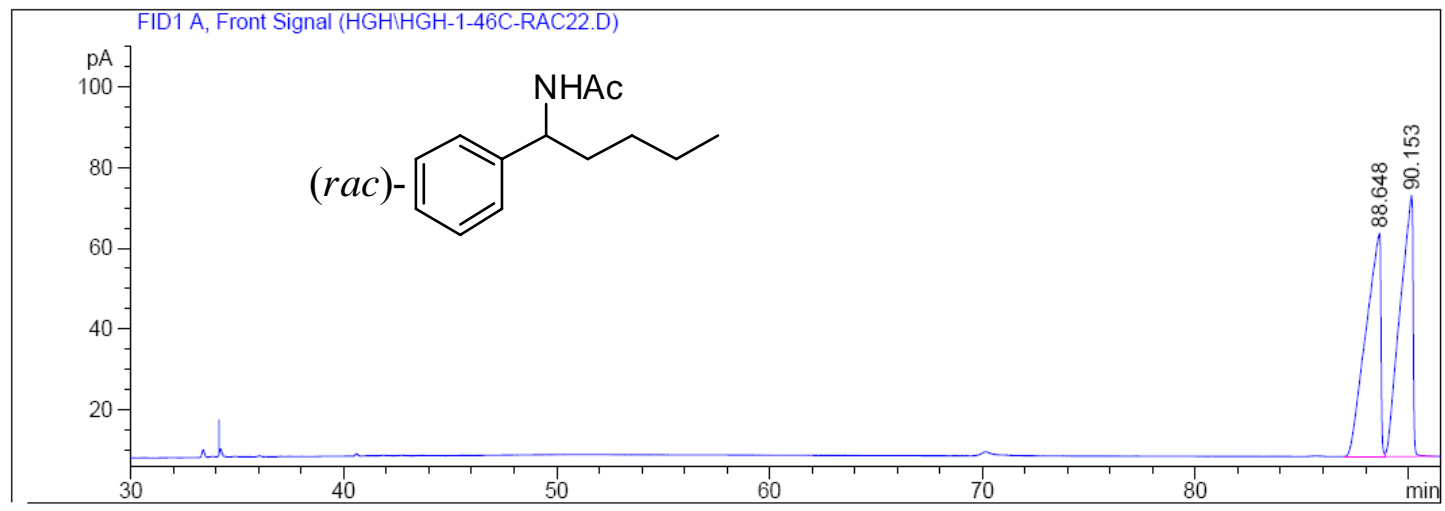



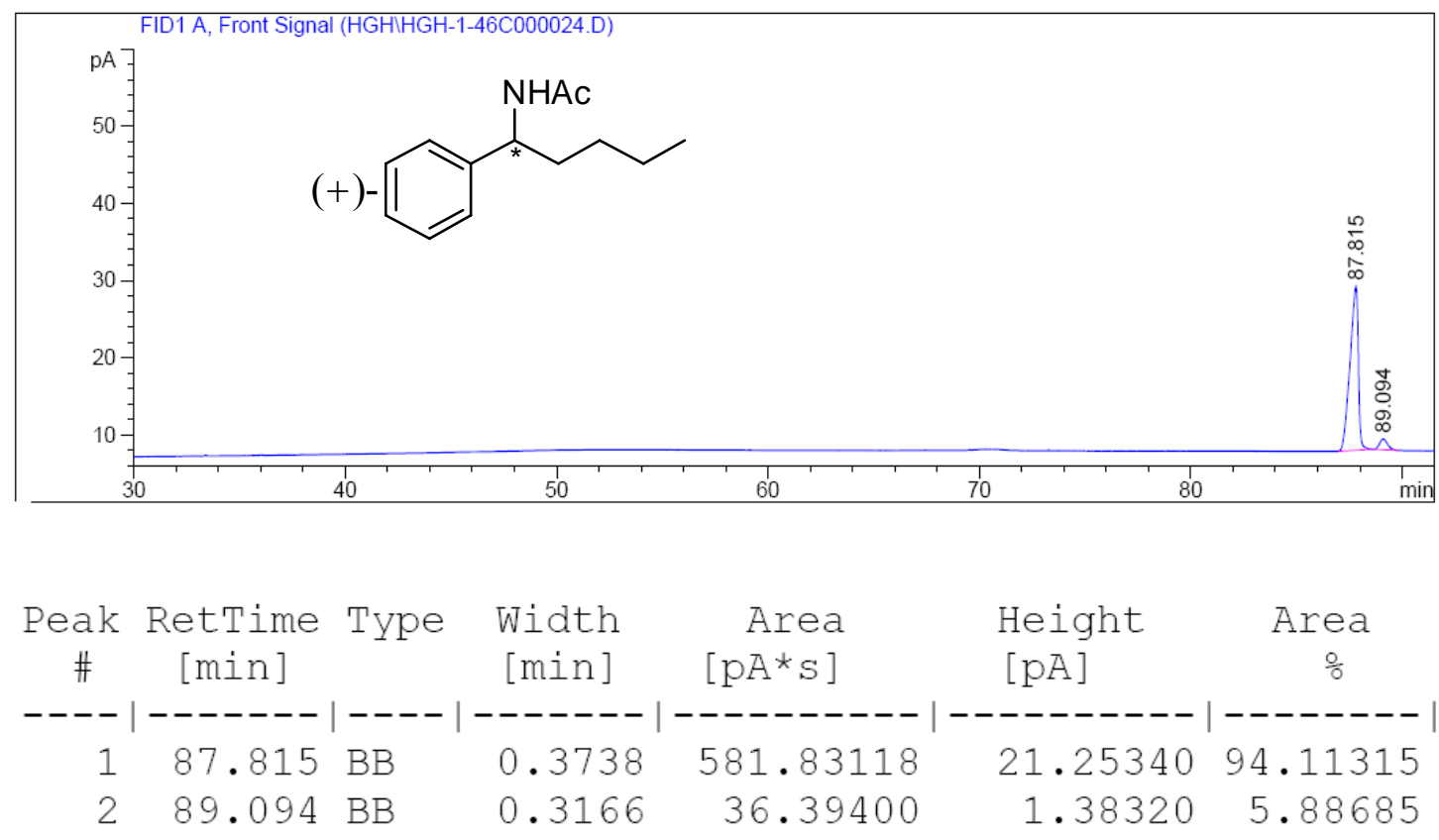

Totals :

$618.22517 \quad 22.63659$
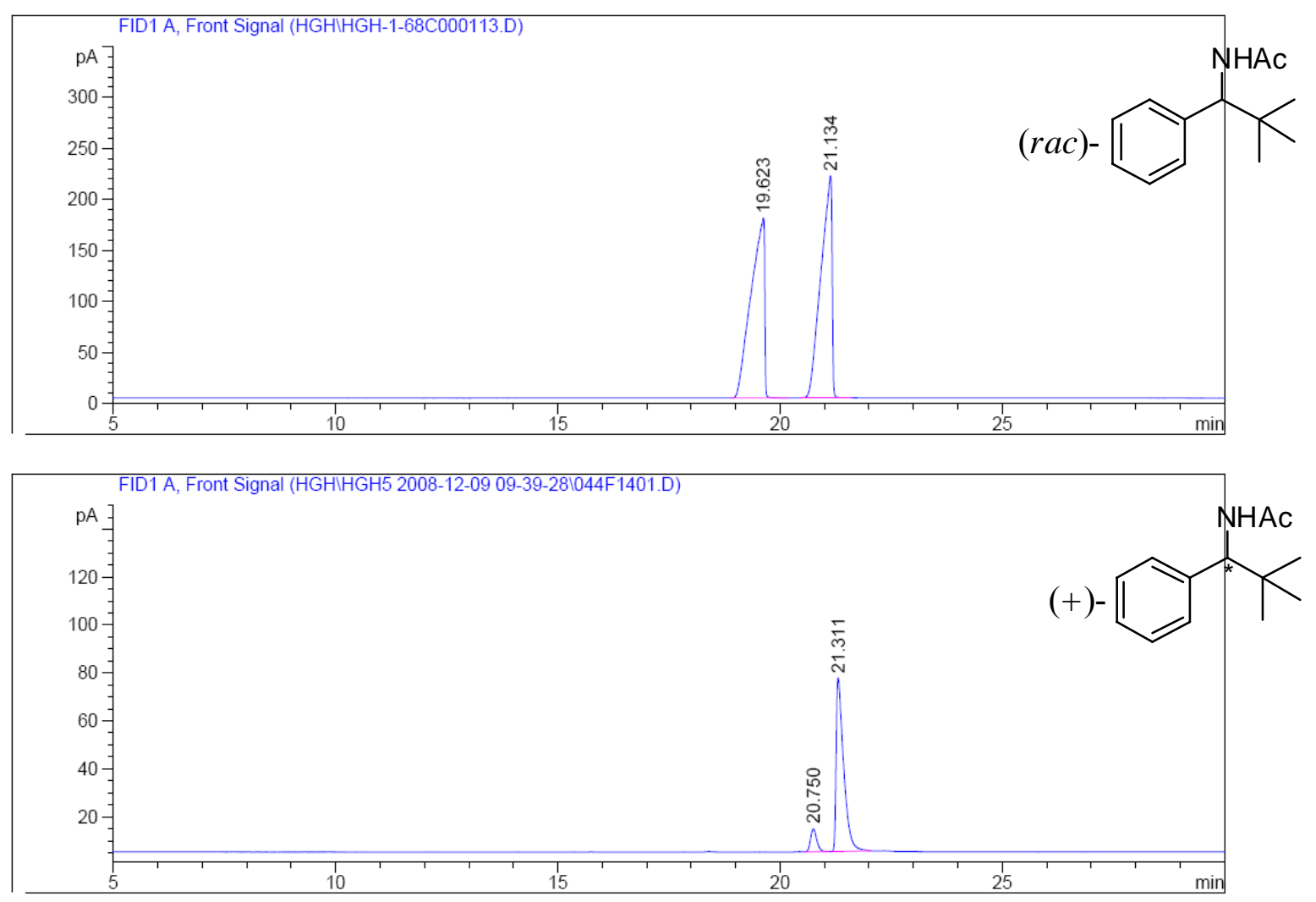


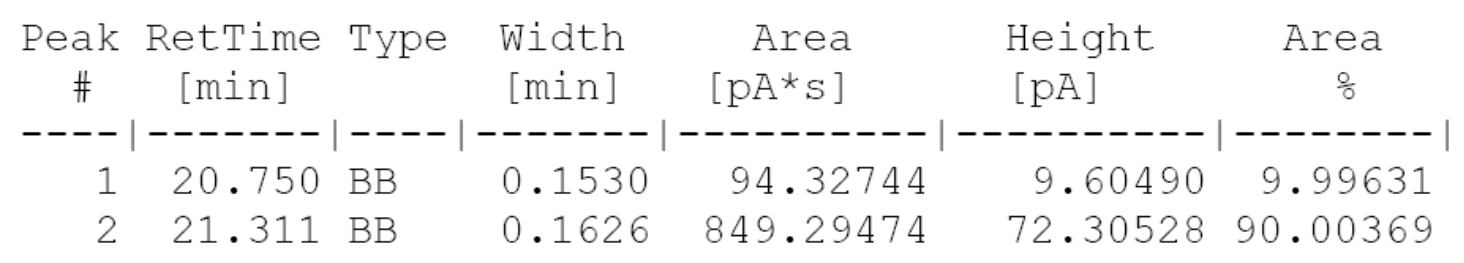

Totals :

$943.62218 \quad 81.91018$
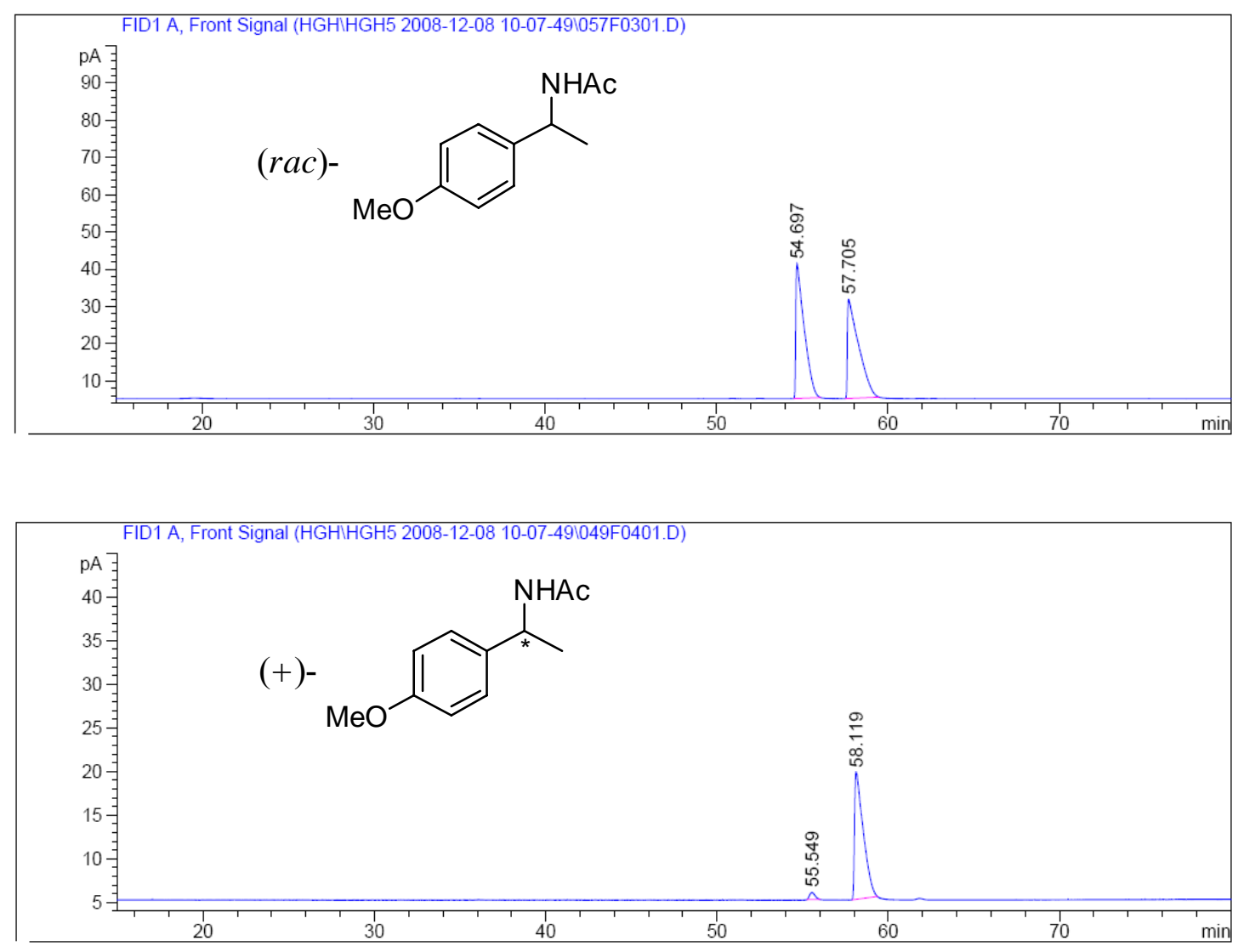

\begin{tabular}{|c|c|c|c|c|c|c|}
\hline $\begin{array}{c}\text { Peak } \\
\#\end{array}$ & $\begin{array}{l}\text { RetTime } \\
\text { [min] }\end{array}$ & Type & $\begin{array}{l}\text { Width } \\
\text { [min] }\end{array}$ & $\begin{array}{r}\text { Area } \\
{\left[\mathrm{pA}^{*} \mathrm{~s}\right]}\end{array}$ & $\begin{array}{l}\text { Height } \\
{[\mathrm{pA}]}\end{array}$ & $\begin{array}{c}\text { Area } \\
\frac{\circ}{0}\end{array}$ \\
\hline-1 & 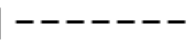 & & & --- & ----- & ------ \\
\hline 1 & 55.549 & $\mathrm{BB}$ & 0.2629 & 18.24997 & $8.19883 e-1$ & 3.42581 \\
\hline 2 & 58.119 & $\mathrm{BB}$ & 0.4169 & 514.47046 & 14.53745 & 96.57419 \\
\hline Total & LS : & & & 532.72043 & 15.35733 & \\
\hline
\end{tabular}



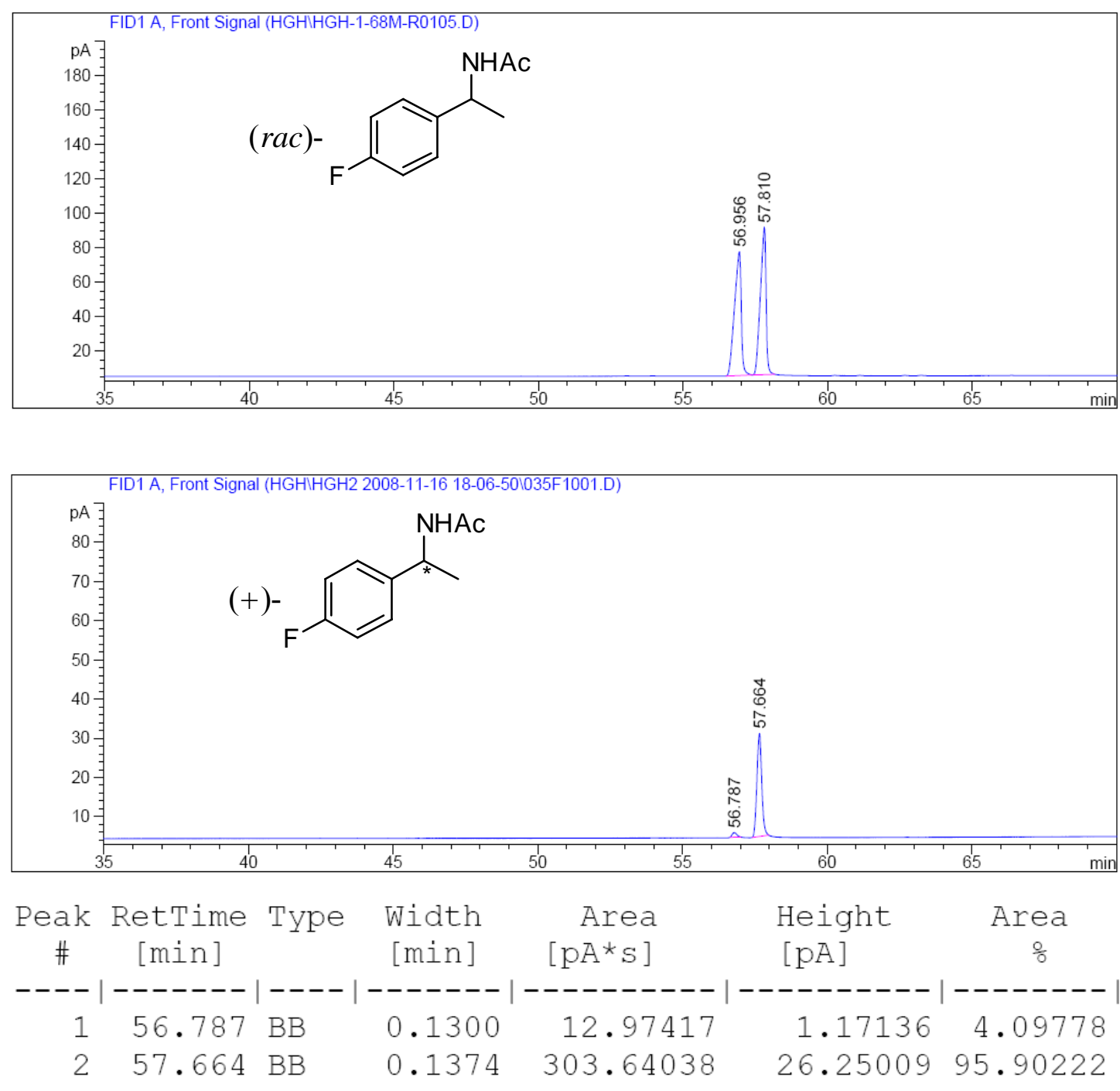
Totals :
$316.61455 \quad 27.42145$

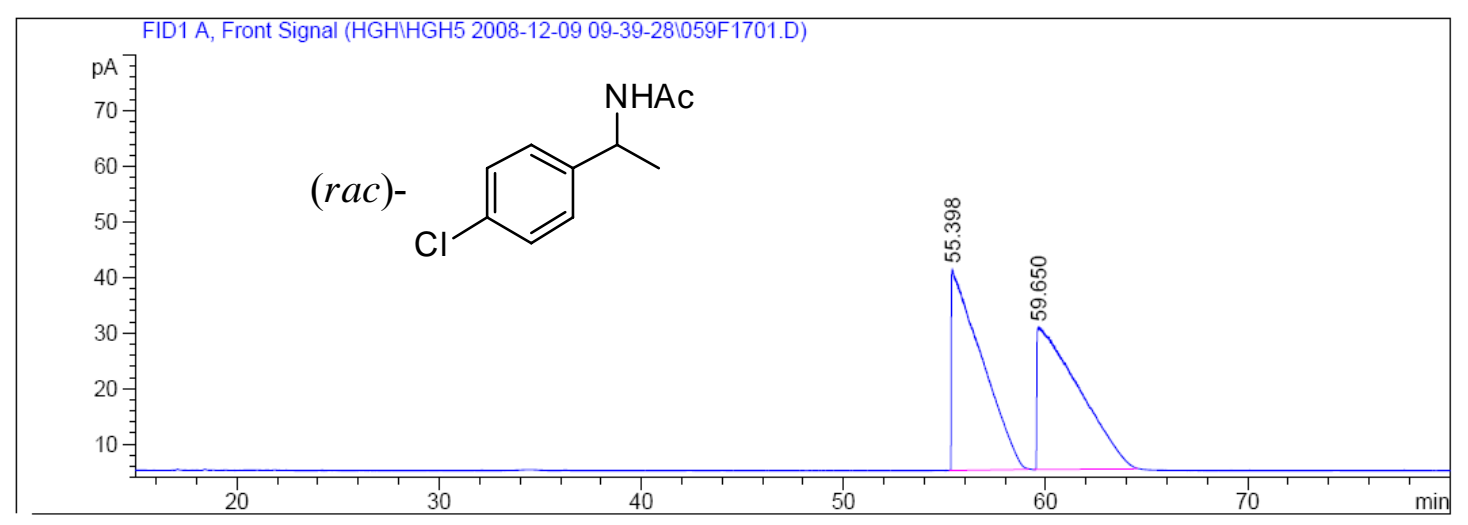



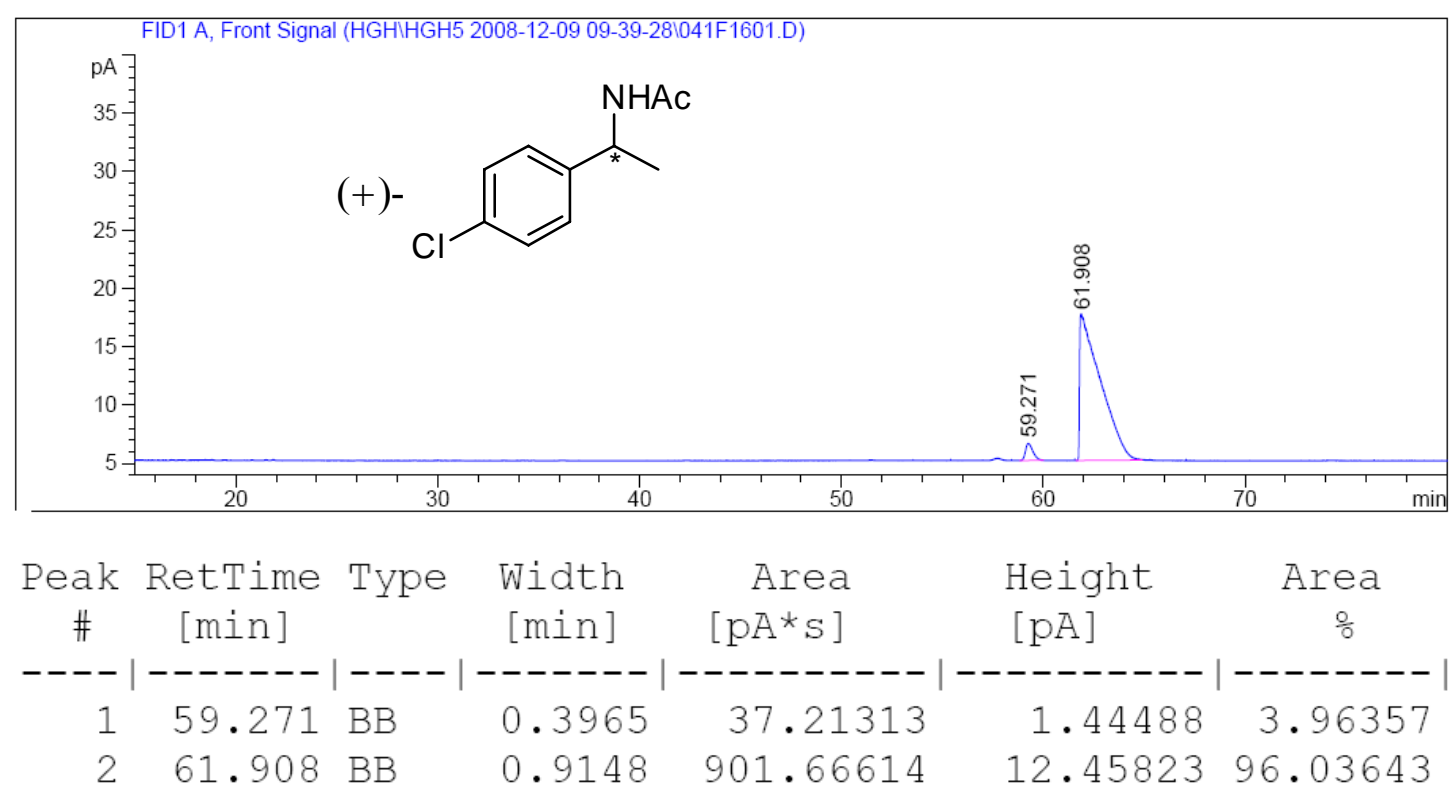

Totals :

$938.87926 \quad 13.90311$
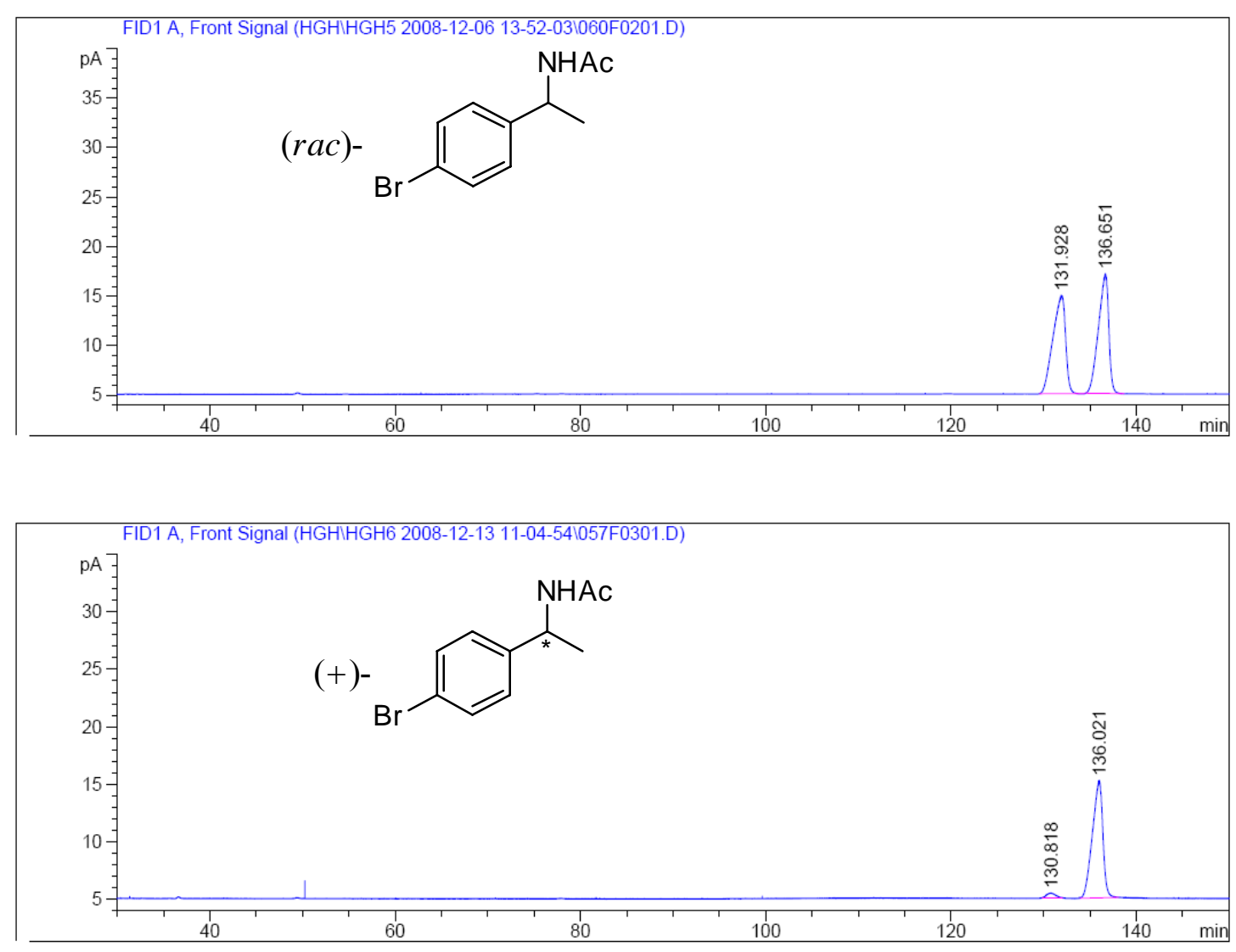


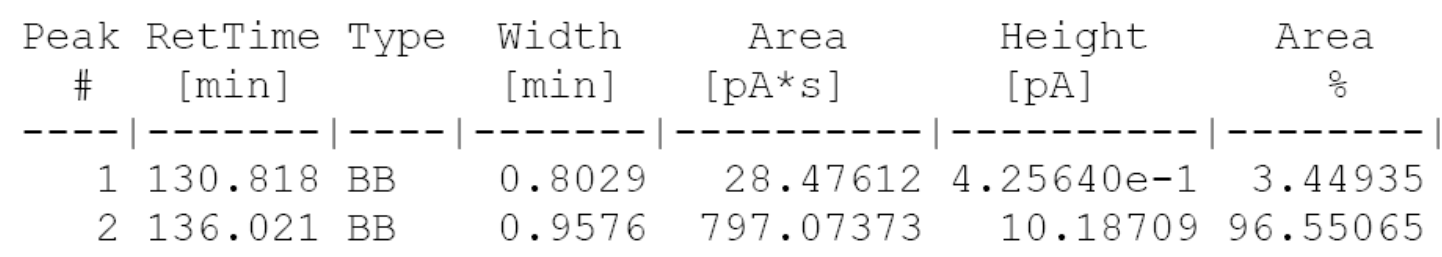

Totals :

$825.54985 \quad 10.61272$
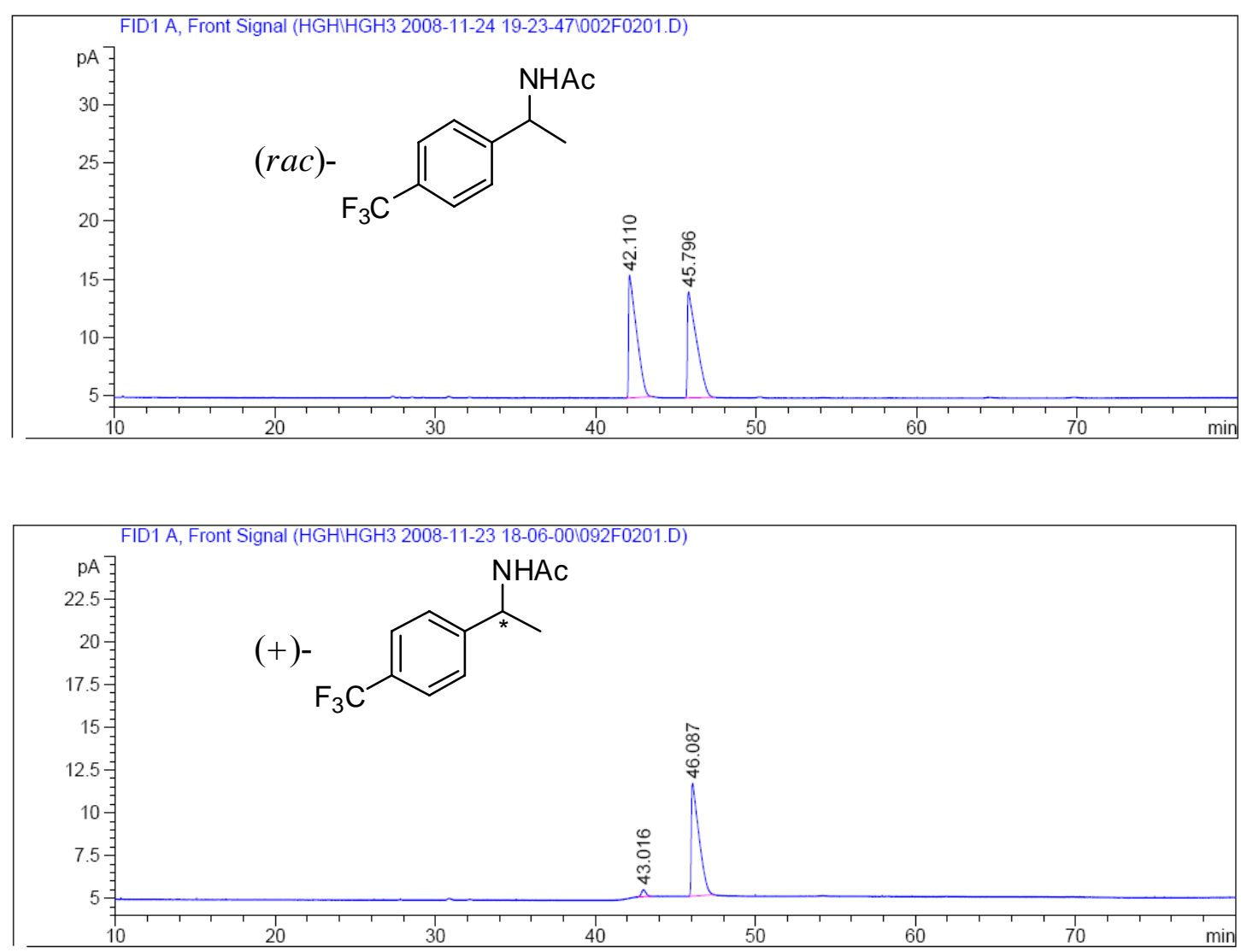

\begin{tabular}{|c|c|c|c|c|c|c|}
\hline $\begin{array}{c}\text { Peak } \\
\quad \#\end{array}$ & $\begin{array}{l}\text { RetTime } \\
\text { [min] }\end{array}$ & Type & $\begin{array}{l}\text { Width } \\
\text { [min] }\end{array}$ & $\begin{array}{r}\text { Area } \\
{\left[\mathrm{pA}^{*} \mathrm{~s}\right]}\end{array}$ & $\begin{array}{l}\text { Height } \\
{[\mathrm{pA}]}\end{array}$ & $\begin{array}{c}\text { Area } \\
\frac{\circ}{0}\end{array}$ \\
\hline---- & & & & ---------- & $1--$ & \\
\hline 1 & 43.016 & BB & 0.2745 & 7.91437 & $4.04750 e-1$ & 816 \\
\hline 2 & 46.087 & $\mathrm{BB}$ & 0.4302 & 217.68459 & 6.56302 & 96.49184 \\
\hline to & & & & 225.59895 & 6777 & \\
\hline
\end{tabular}



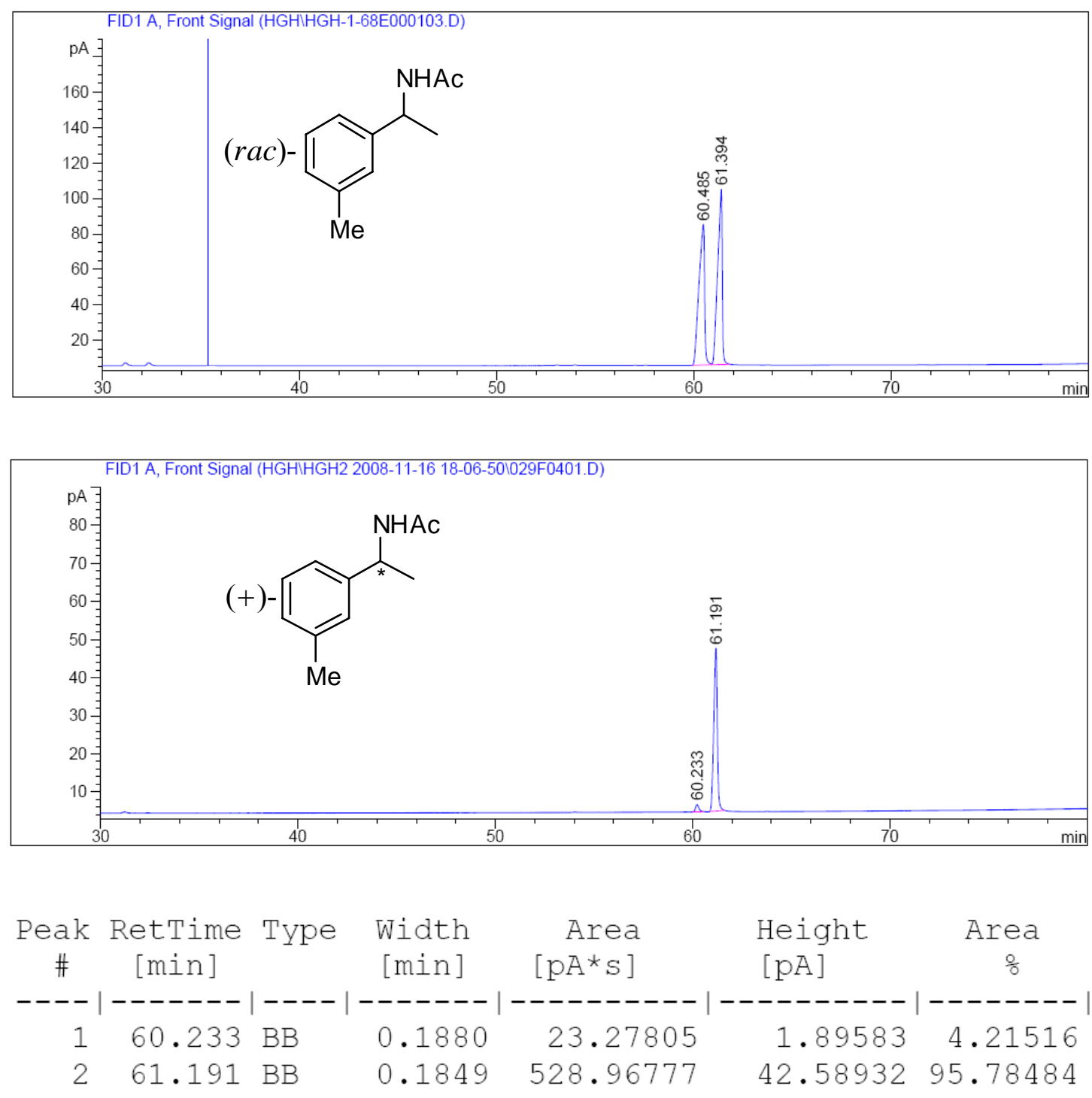

Totals :

$552.24582 \quad 44.48515$

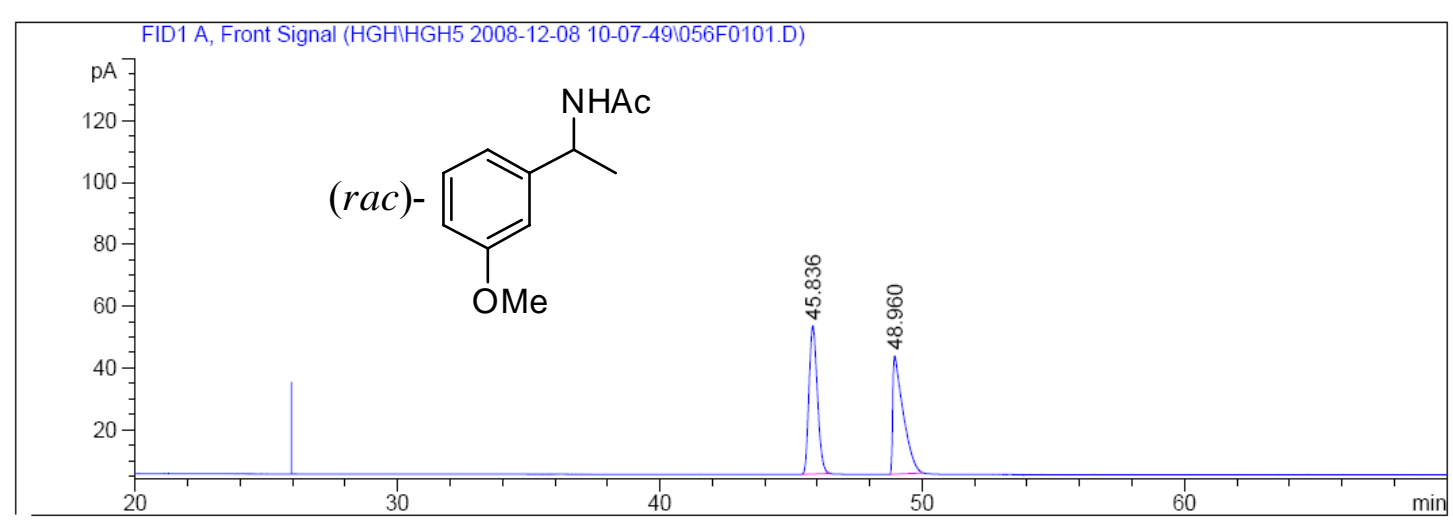



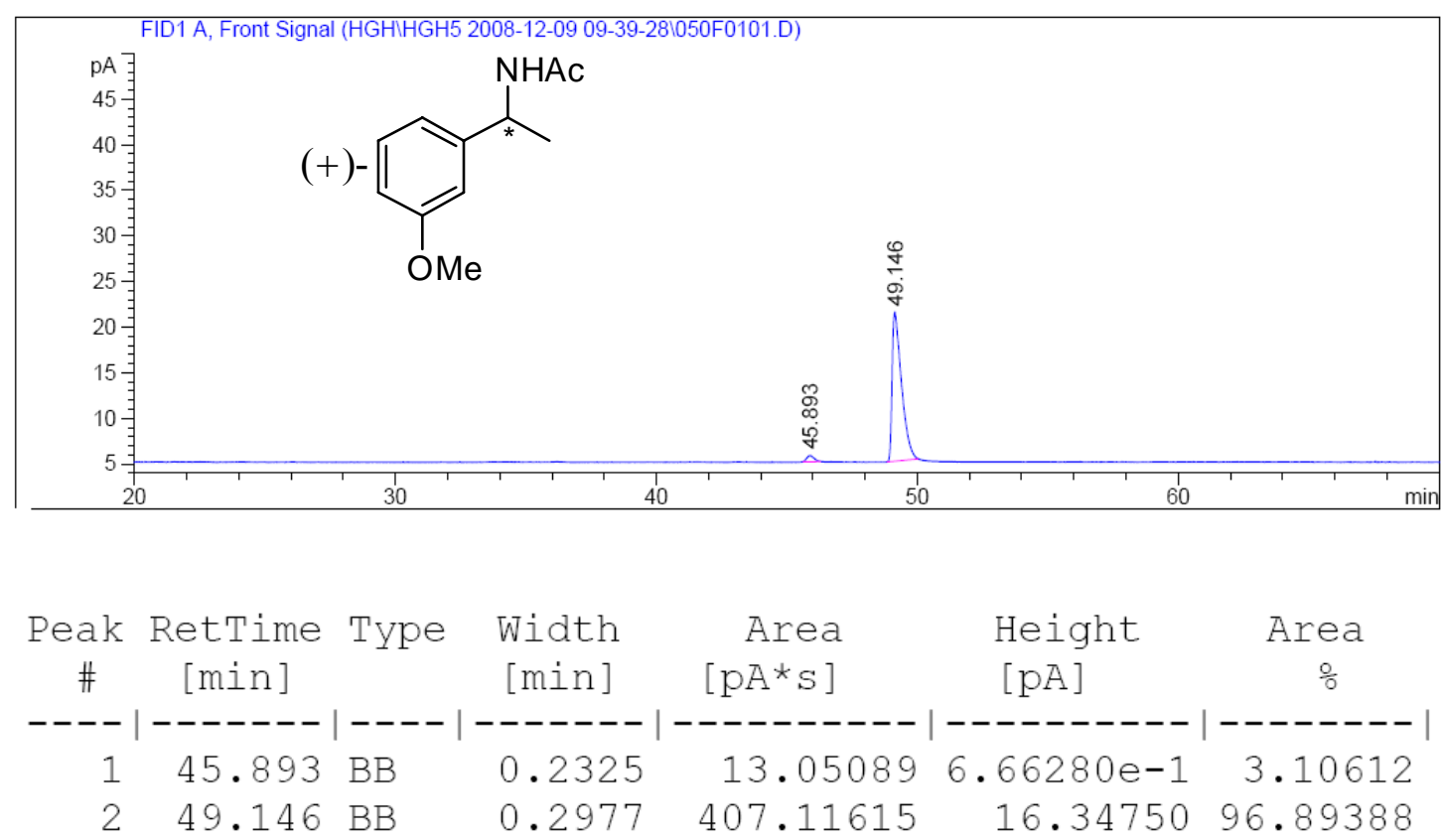

Totals :

$420.16704 \quad 17.01378$
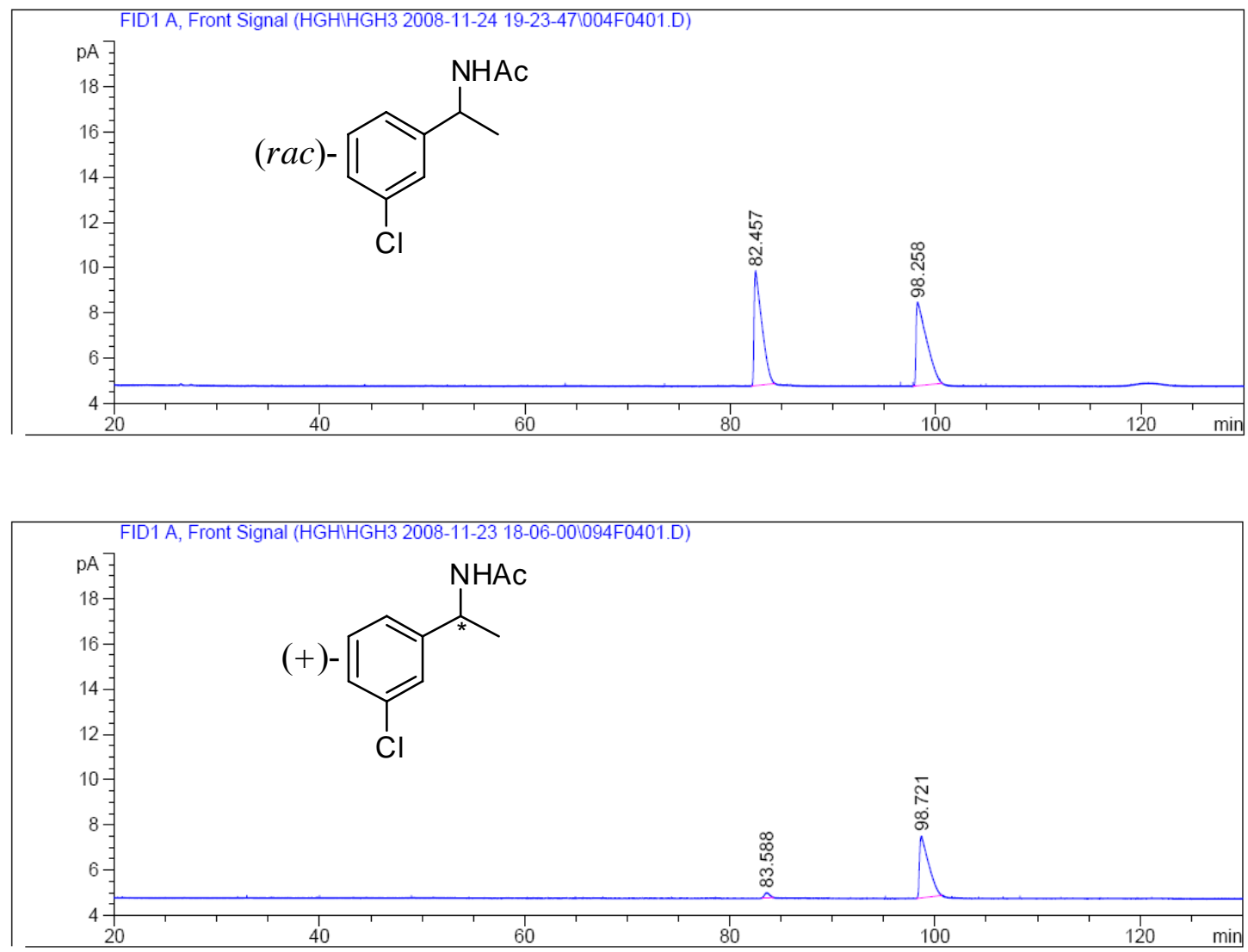


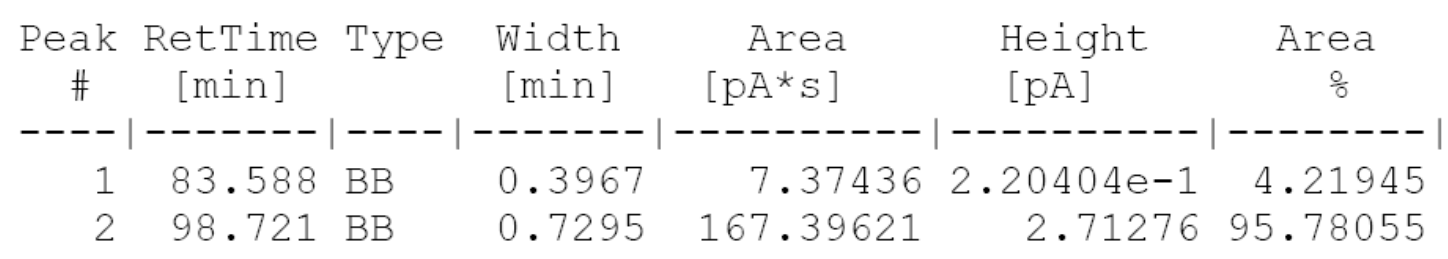
Totals :
174.77057
2.93317
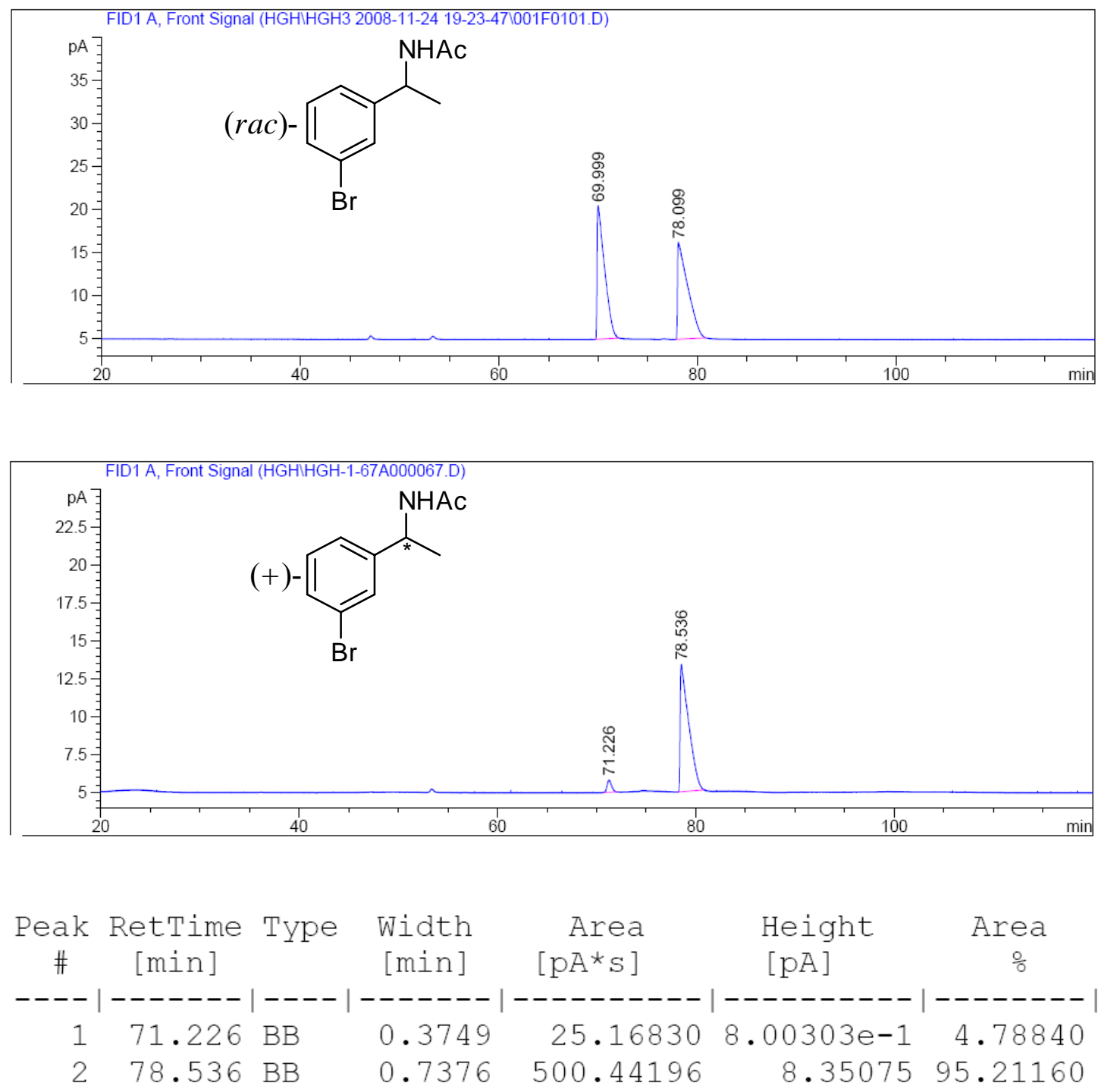

Totals :

$525.61025 \quad 9.15106$ 

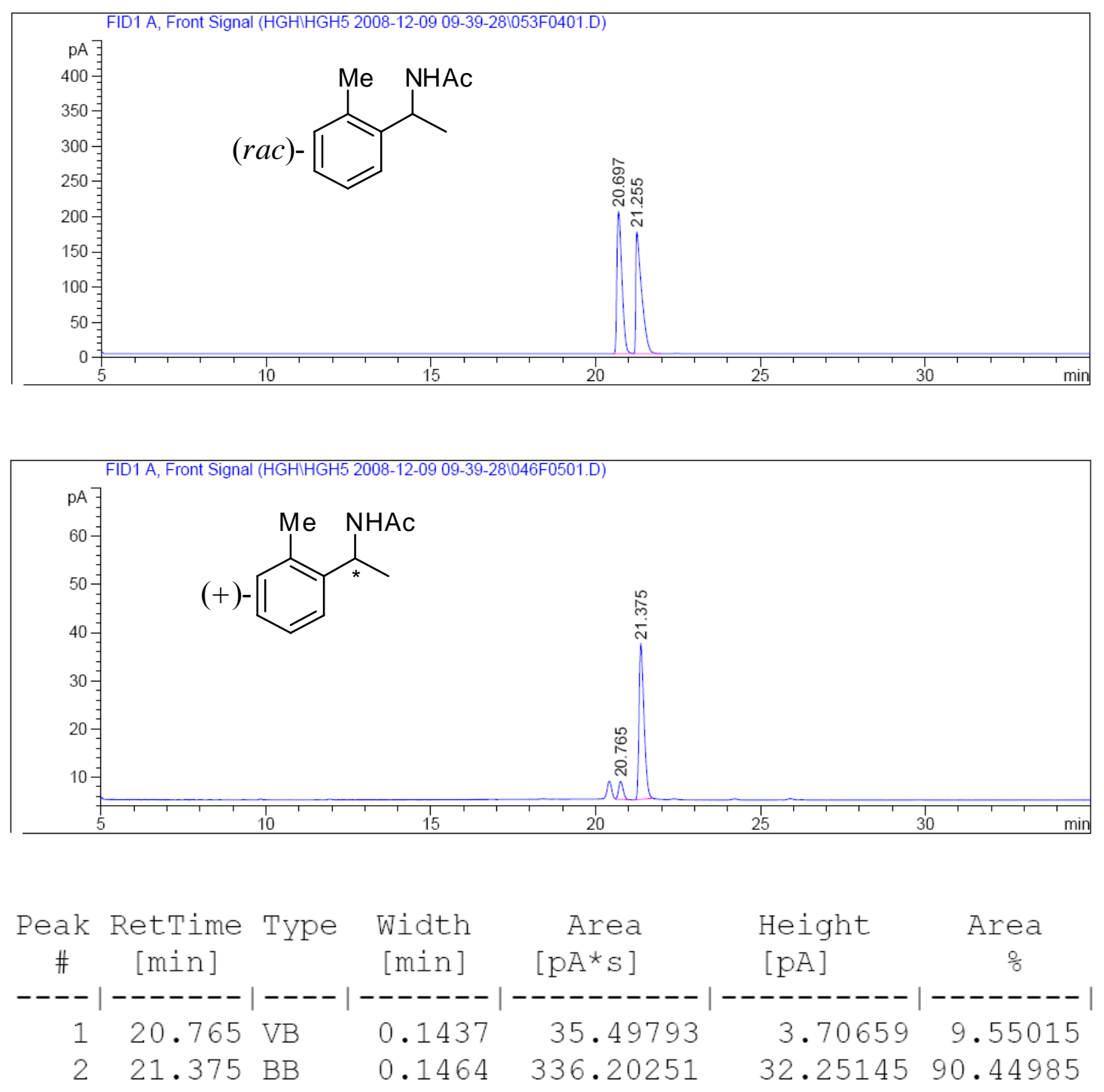

Totals :

$371.70045 \quad 35.95805$

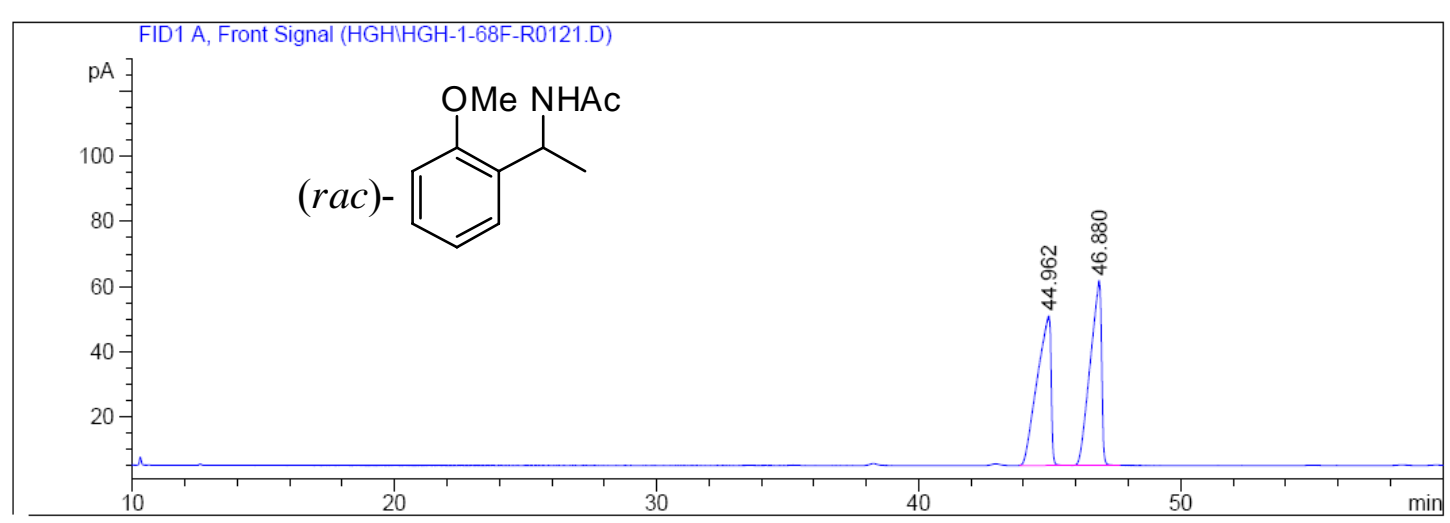



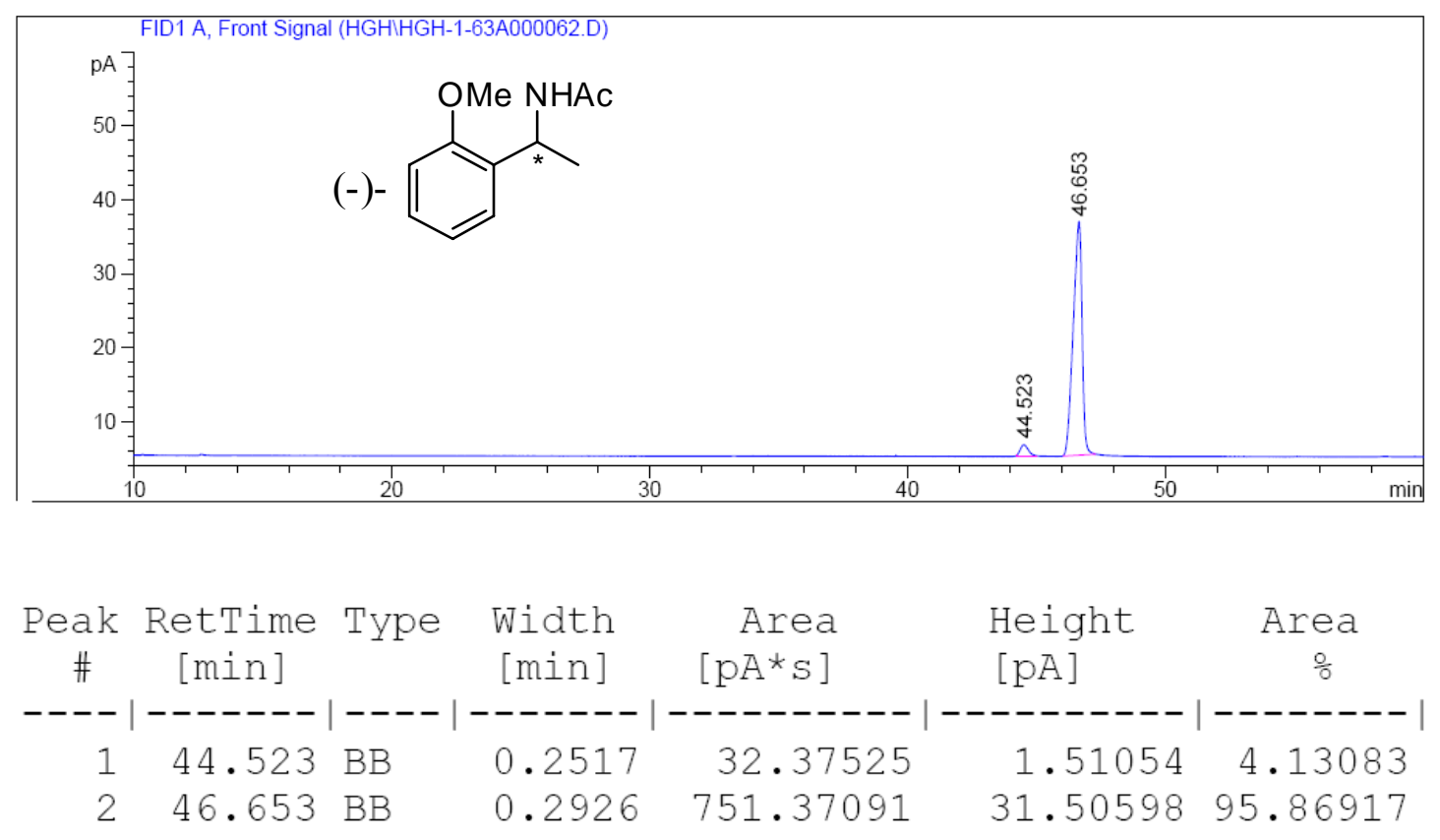

Totals :

$783.74616 \quad 33.01652$
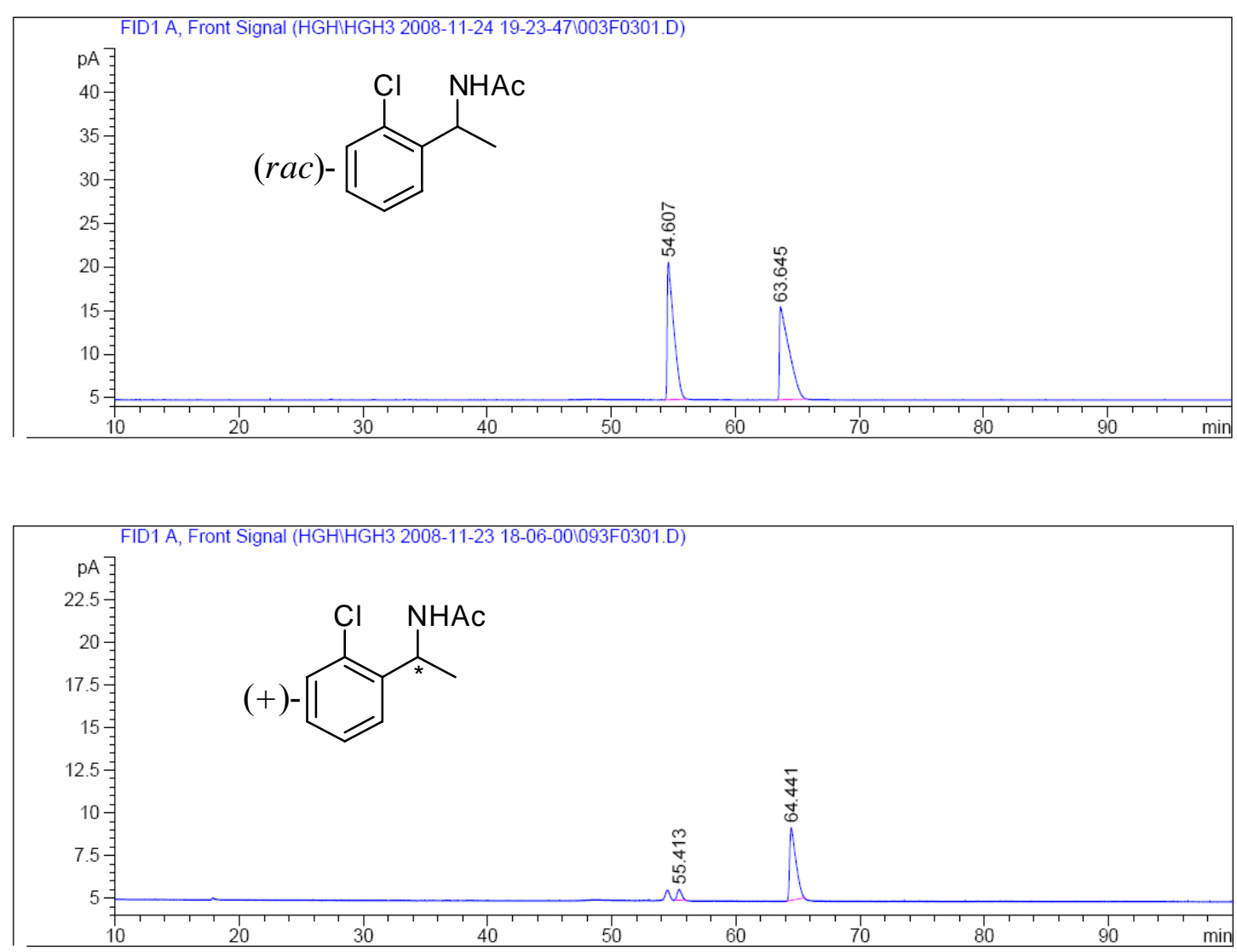

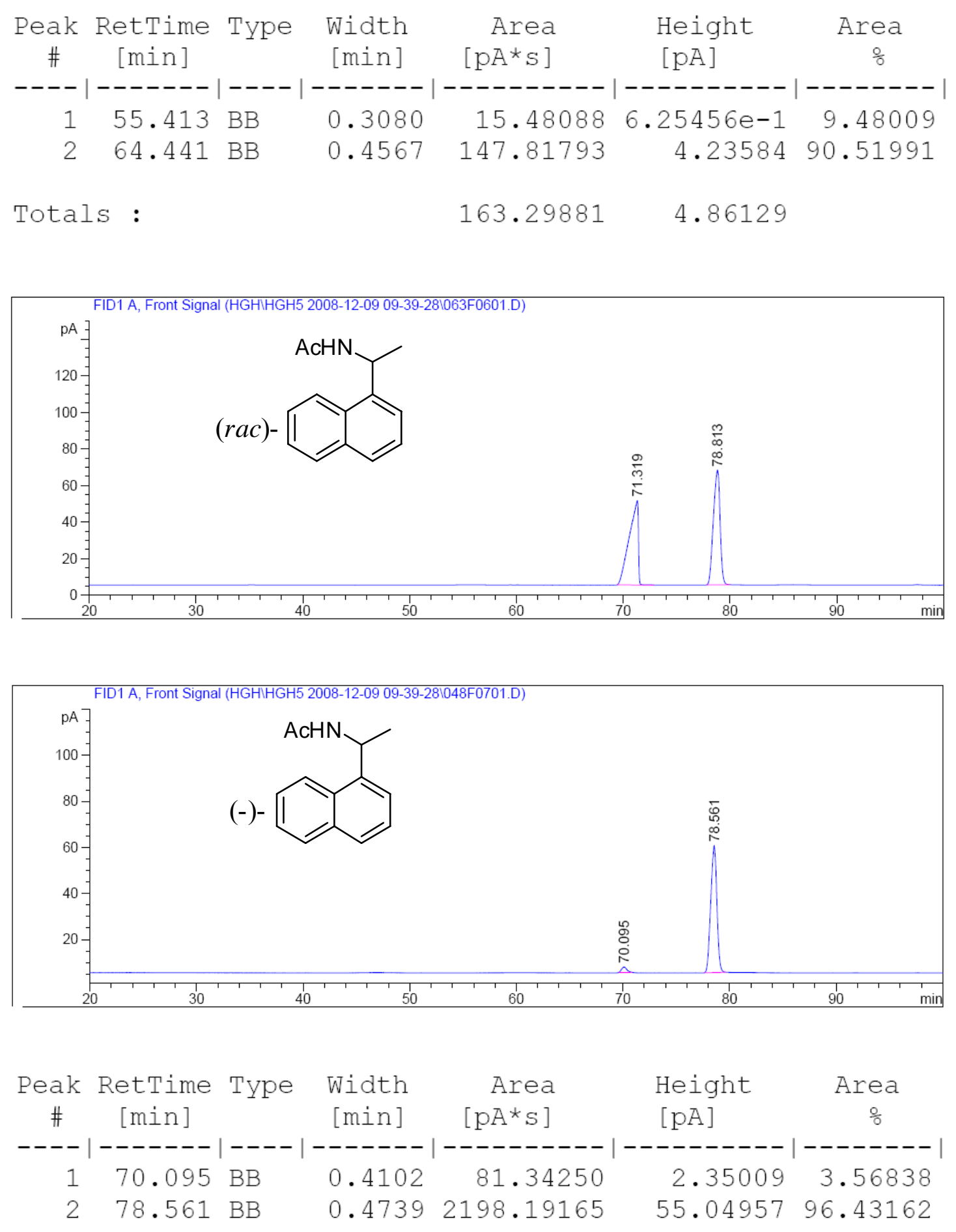

Totals : $\quad 2279.53415 \quad 57.39966$ 

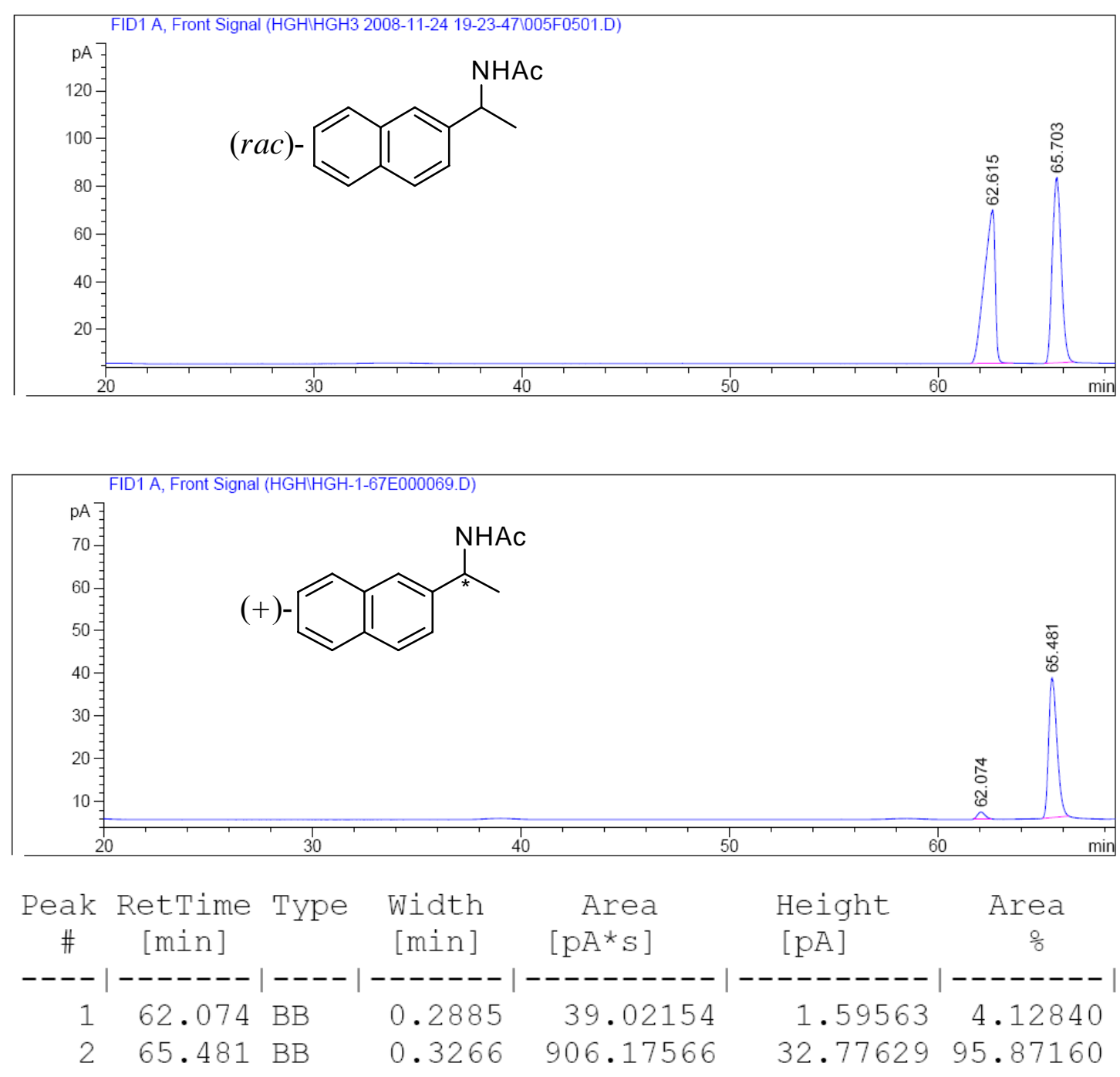

Totals :

$945.19720 \quad 34.37192$

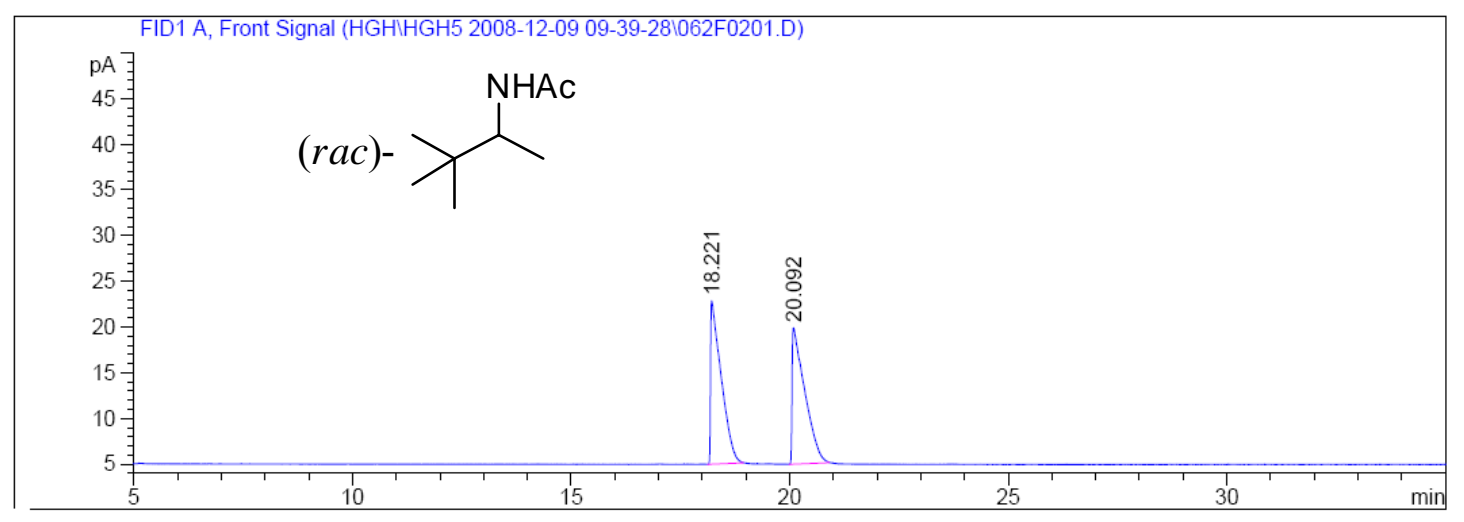



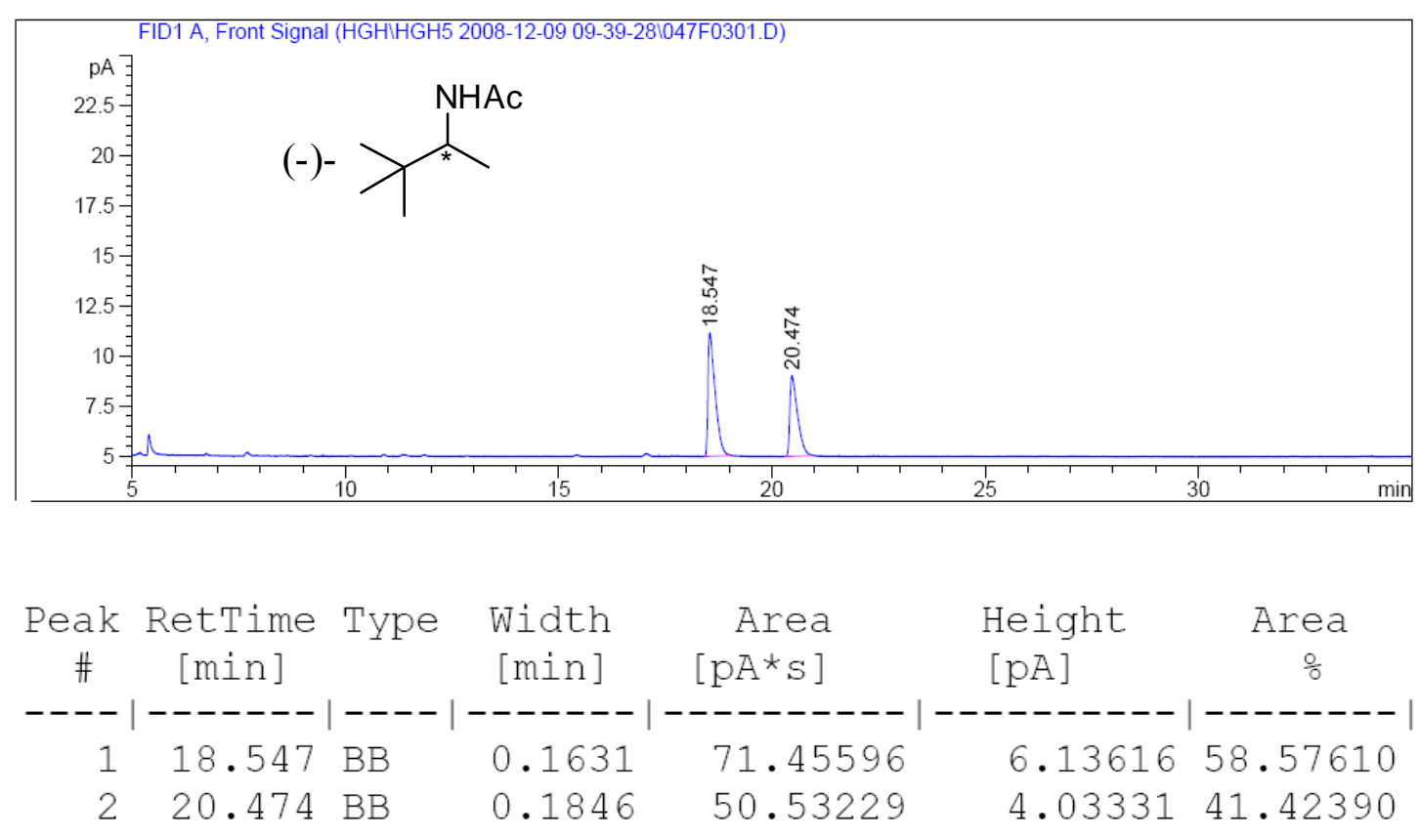

Totals :

$121.98824 \quad 10.16947$
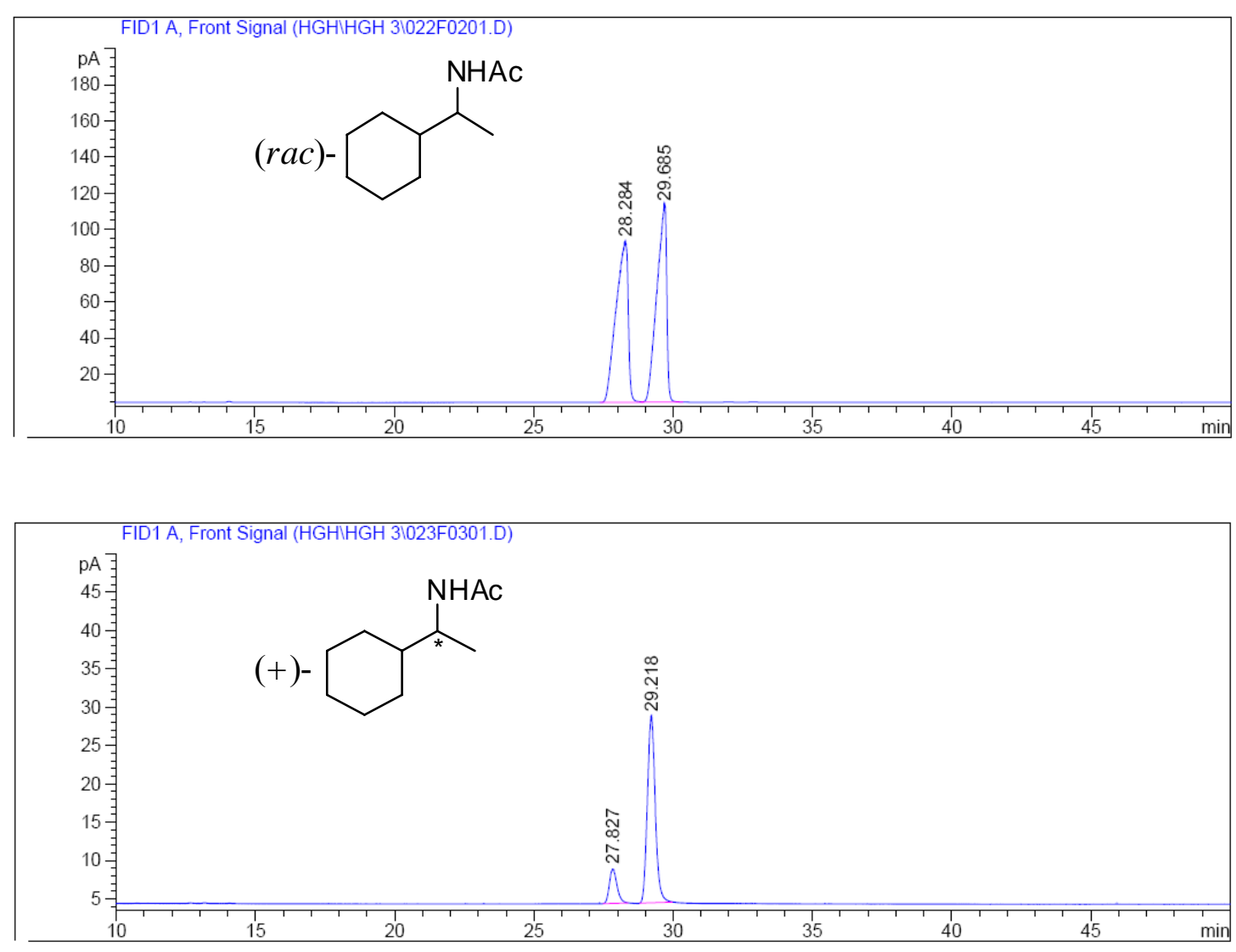


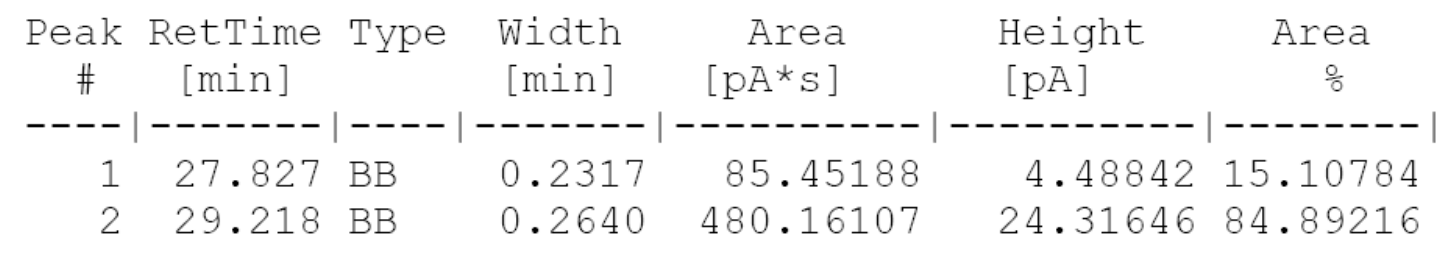

Totals :

$565.61295 \quad 28.80488$
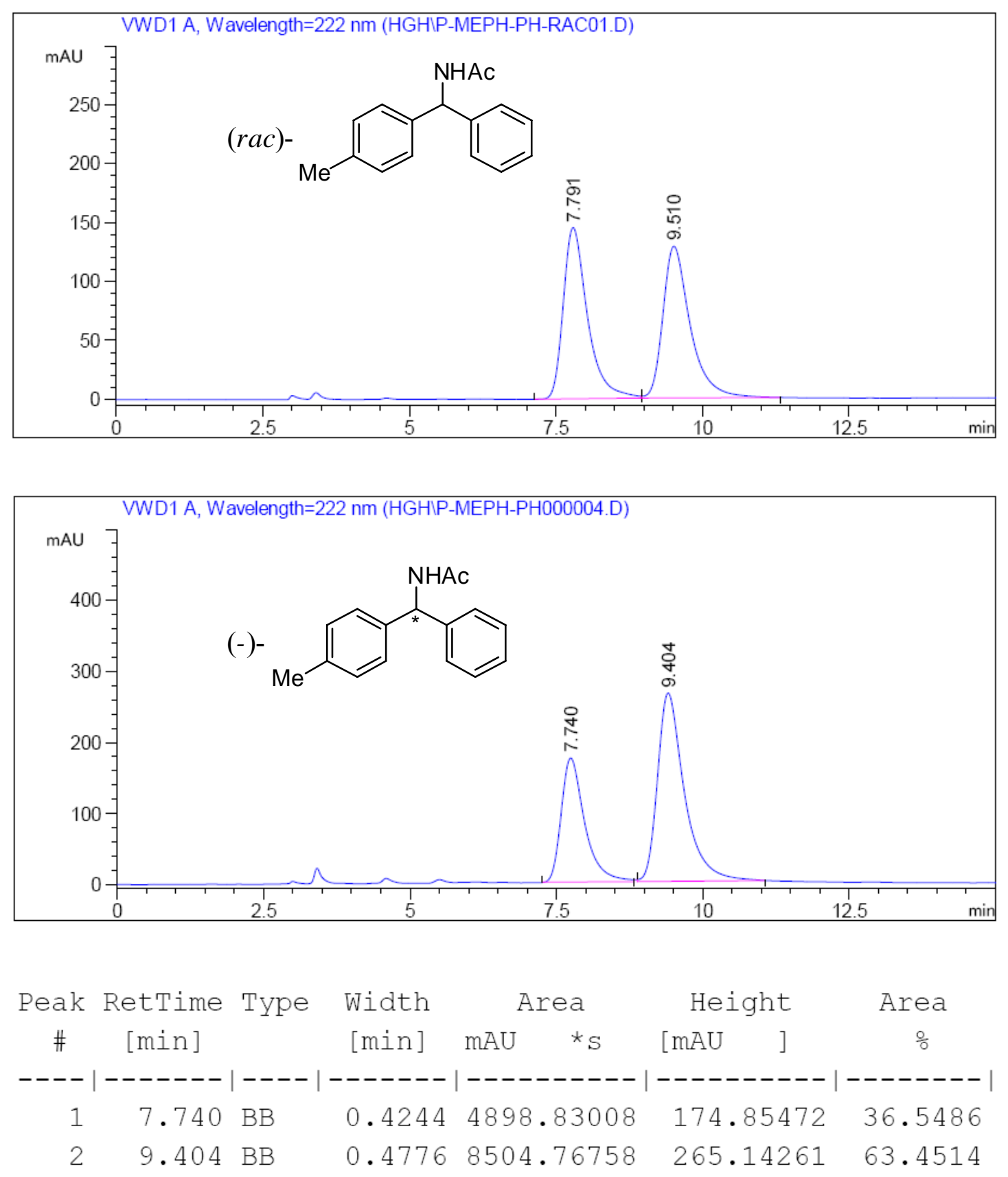

Totals :

$1.34036 \mathrm{e} 4 \quad 439.99733$ 
Acknowledgement. The authors thank Dr. Rong Xiang for chiral HPLC analysis of (R)- $\alpha$-4-dimethylbenzenemethanol.

(1) Boyd, D. R.; McCombe, K. M.; Sharma, N. D. J. Chem. Soc., Perkin Trans. I1, 1986, 867.

(2) Barluenga, J.; Gonzalez, F. J.; Carlon, R. P.; Fustero, S. J. Org. Chem., 1991, 56, 6751.

(3) Smith, H. E.; Neergaard, J. R.; Paulis, T. D.; Chen, F.-M. J. Am. Chem. Soc. 1983, 105, 1578.

(4) Boezio, A. A.; Solberghe, G.; Lauzon, C.; Charette, A. B. J. Org. Chem. 2003, 68, 3241.

(5) Mukade, T.; Dragoli, D. R.; Ellman, J. A. J. Comb. Chem. 2003, 5, 590.

(6) Andrés, C.; Nieto, J.; Pedrosa, R.; Villaman, N. J. Org. Chem. 1996, 61, 4130.

(7) Shi, M.; Lei, Z.-Y.; Xu, Q. Adv. Synth. Catal. 2006, 348, 2237.

(8) Colyer, J. T.; Andersen, N. G.; Tedrow, J. S.; Soukup, T. S.; Faul, M. M. J. Org. Chem. 2006, 71, 6859.

(9) Allison, B. D; Hack, M. D.; Phuong, V. K.; Rabinowitz, M. H.; Rosen, M. D. US Patent Appl. Publ. US 2005038032.

(10) Küendig, E. P.; Botuha, C.; Lemercier, G.; Romanens, P.; Saudan, L.; Thibault, S. Helv. Chim. Acta 2004, 87, 561.

(11) Krzyzanowska, B. Synthesis 1982, 4, 270.

(12) Plobeck, N.; Powell, D. Tetrahedron: Asymmetry 2002, 13, 303. 